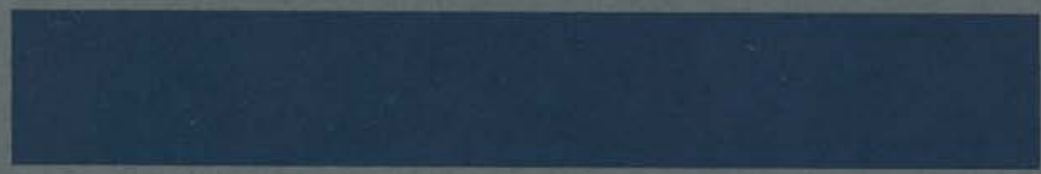

\title{
BURNUP DATA
}

FROM UO ${ }_{2}-0.48$ wt $\% \mathrm{PuO}_{2}$ FUEL

R. P. Matsen

August 1970

\section{AEC RESEARCH \& DEVELOPMENT REPORT}




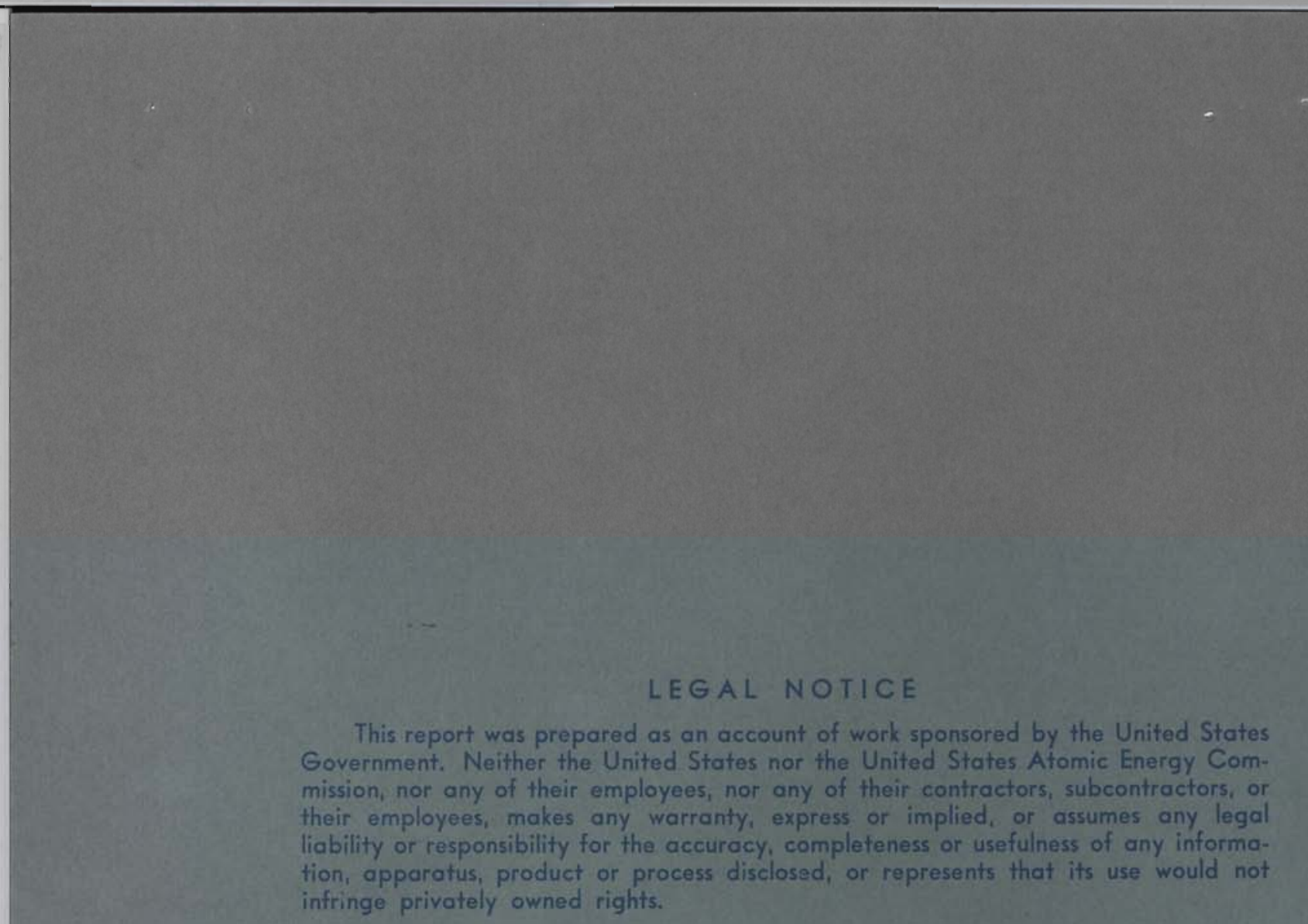

Government, Neither the United States nor the United States Atomic Energy Commission, nor any of their employees, nor any of their contractors, subcontractors, or their employees, makes any warranty, express or implied, or assumes any legal ty or responsibility for the accurecy somplete infringe privately owned rights.

\section{PACIFIC NORTHWEST LABORATORY \\ RICHLAND, WASHINGTON \\ operated by \\ BATTELLE MEMORIAL INSTITUTE _. \\ for the}

UNITED STATES ATOMIC ENERGY COMMISSION UNDER CONTRACT AT(45-1)-1830 


\section{ERRATA}

The headings have been omitted from pages $\mathrm{A}-30, \mathrm{~A}-32$ and $\mathrm{A}-56$. They should read

$\underline{\text { Page }}$

A-30

A-32

A-56

\section{Heading}

Element 5185 Analysis of the Sample 23.50 inches from the top of the center rod

Element 5185 Analysis of the Sample 38.00 inches from the top of the center rod

Element 5185 Analysis of the Sample 34.00 inches from the top of the outside rod 
BNWL -1488

UC-80, Reactor

Technology

- BURnuP DATA FROM $\mathrm{UO}_{2}-0.48$ wt\% $\mathrm{PuO}_{2}$ FUEL

By

R. P. Matsen

Reactor Physics Department

Physics and Engineering Division

August 1970

BATTELLE MEMORIAL INSTITUTE

PACIFIC NORTHWEST LABORATORIES

RICHLAND, WASHINGTON 99352 
Printed in the United States of America Available from

Clearinghouse for Federal Scientific and Technical Information National Bureau of Standards, U.S. Department of Commerce Springfield, Virginia 22151

Price: Printed Copy $\$ 3.00 ;$ Microfiche $\$ 0.65$ 


\section{$\underline{\text { ABSTRACT }}$}

Burnup data obtained from the destructive analysis of $\mathrm{UO}_{2}-0.48$ wt\% $\mathrm{PuO}_{2}$ fuel irradiated in the Plutonium Recycle Test Reactor are presented. The measured data are partially analyzed, however no effort has been made to detect questionable data. The data should be evaluated before it is used further. 
,

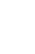

-

.

- 
$\underline{\text { BURNUP DATA FROM UO }} 2-\frac{-0.48 \omega t \% \text { PuO }}{2} 2$ FUEL

R. P. Matsen

\section{INTRODUCTION}

Burnup data obtained as the result of the destructive analysis of $\mathrm{Ua}_{2}-0.48$ wt\% $\mathrm{PuO}_{2}$ fuel irradiated in the Plutonium Recycle Test Reactor (PRTR) are presented in this report. The samples were cut from elements which were the first of a series of mixed urania-plutonia fuels irradiated in the PRTR. The most exposed sample has a burnup of approximately $6500 \mathrm{MWd} / \mathrm{MTM}$. The data that was measured for each sample is recorded in this report and has been used to calculate quantities that should be more useful to the analyst. One standard deviation uncertainties of the measured and calculated quantities are also included. At the time the measurements were made, radiochemical techniques were used. The more accurate isotopic dilution techniques currently in use had not yet been developed. Because no effort has been made to locate or mark questionable data, the validity of the data should be evaluated before it is used.

\section{Fuel Description}

The pre-irradiated fuel was natural uranium di-oxide enriched with $\mathrm{PuO}_{2}$. Nominally, the $\mathrm{Pu} / \mathrm{U}$ ratio was equal to 0.0048 by weight. The plutonium atom percents at the time the irradiation began were $80.183 / 17.273 / 2.310 / 0.234$ for ${ }^{239} \mathrm{Pu} /{ }^{240} \mathrm{Pu} /{ }^{241} \mathrm{Pu} /{ }^{242} \mathrm{Pu}$ respectively. The fuel rods possess an active fuel length of 88.7 inches and the fuel diameter is 0.5 inches. The cladding material is Zircaloy II. Three rods from each of seven nineteenrod PRTR clusters were loaded with pelletized fuel. One was the center rod of the cluster, another was located in the middle or six rod ring of the cluster, and the other was positioned in the outside or 12 rod ring of the cluster. Burnup samples were cut from pelletized rods. The remaining rods 
within each cluster were, filled with $\mathrm{UO}_{2}-\mathrm{PuO}_{2}$ powder and were compacted by a swaging process. No burnup samples were obtained from these swaged rods. Two 19-rod fuel elements have been sampled. Their irradiation history is shown in Table I.

$\underline{\text { TABLE I }}$

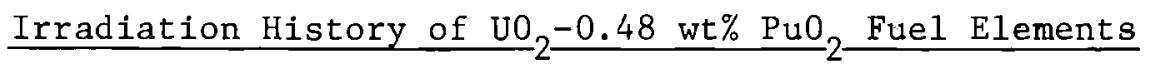

Element

5187

5185
Element Average

Exposure (MWd)

100.2

245.7

In

May 28, 1963

May 28, 1963
Out

Nov. 24,1963

Oct. 9,1964

\section{Measured Data}

The following data were measured or recorded for each fuel sample.

1. Position of the sample within the rod

2. Weight of the fuel sample plus cladding material

3. Weight of the clad after the fuel has been dissolved

4. Volume of the solution in which the fuel was dissolved

5. Time from date of fuel discharge to date of analycis.

6. Uranium atom ratios: ${ }^{234} \mathrm{U} /{ }^{238} \mathrm{U},{ }^{235} \mathrm{U} /{ }^{238_{\mathrm{U}}}$, and ${ }^{236} \mathrm{U} /{ }^{238} \mathrm{U}$

7. Plutonium atom ratios: ${ }^{240} \mathrm{Pu} /{ }^{239} \mathrm{Pu},{ }^{241} \mathrm{Pu} /{ }^{239} \mathrm{Pu}$, and ${ }^{242} \mathrm{Pu} /{ }^{239} \mathrm{Pu}$

8. Plutonium decays measured as decays per minute per milliliter of solution

9. Percent of plutonium decays caused by ${ }^{238} \mathrm{Pu}$

10. Cesium- 137 decays measured as decays per minute per milliliter of solution. 
One standard deviation uncertainties were estimated for measurements $6,7,8$, and 10. The other uncertainties were assumed to be negligible.

Additional data has been acquired for some of the samples and they are recorded in Table II. These data consist of the amount of uranium and/or plutonium in solution for a given sample. Coulometric and isotopic dilution techniques were employed in obtaining these measurements. Some gamma scan information was obtained with a NaI crystal for the rods from element 5187. These are presented in Table III. The measurements were made at positions which progress in a direction from the top to the bottom of the rod. The fuel begins at a different (but unknown) position for each of the rods. The background counts were measured at some of the positions and these are also shown.

Interpretation of the Data

The results for each sample are presented in the Appendix which consists of the output of the PRGTRY burnup analysis code. In addition to the measured data, other quantities derived from the data are also presented. A quantity enclosed in parenthises is the one standard deivation uncertainty of the preceding number. These uncertainties are calculated from the uncertainties assigned to the measured data by standard propagation of error techniques. Most of the headings are self explanatory. The symbol BOL means Beginning of Life (i.e., at the time that the element was first charged) and EOL means End of Life (i.e., at the time the element was discharged). The term A/O means atom percent and GM stands for gram. All quantities that are reported in units of grams are normalized to one gram of uranium plus plutonium at the Beginning of Life (notice that the total BOL uranium plus the total BOL plutonium adds up to one gram). 
Parameters that are used in the reduction of the measured data are also shown along with the rest of the information for each sample. Under the heading, SPECIFIC ACTIVITY (D/M/ML), the alpha activities are listed in decays per minute for one gram of each of the various plutonium isotopes. Parameters needed for calculating the fissions and burnup in Megawatt Days per Metric Ton of heavy Metal (i.e., MWD/MTM) are the ${ }^{137}$ Cs fission yields, the number of fissions (DEL) that occur in ${ }^{238} \mathrm{U}$ because of fast neutrons from the other fissionable isotopes, and the MEV/FISSION for each of the fissionable isotopes. Other parameters used in the calculations are listed under ASSUMPTIONS.

The plutonium atom ratios for the samples from the positions at $34.0,38.5$, 45.25, and 65.0 inches of the outside ring rod of element 5185 were not measured. The plutonium atom ratios used in the Appendix for these samples was the same as for the samples from the same rod at positions of $33.5,38.0$, 44.75, and 64.5 inches respectively. 


\section{ACKNOWLEDGMENTS}

The measurements were supervised by R.D. Elder (Radiometallurgy Laboratory), A.C. Leaf (Chemical Analysis Laboratory), and Dr. C.R. Lagergren (Mass Spectrometer Laboratory). P.E. Coudron and E.B. Reppond assisted in the analysis of the data. 


\section{TABLE II}

\section{Additional Experimental Measurements}

\begin{tabular}{|c|c|c|c|c|c|c|}
\hline \multirow[b]{2}{*}{ Element } & \multirow{2}{*}{$\begin{array}{l}\text { Ring that } \\
\text { Rod is from }\end{array}$} & \multirow{2}{*}{$\begin{array}{l}\text { Position } \\
\text { (inches) }\end{array}$} & \multicolumn{2}{|c|}{ Coulometer } & \multirow{2}{*}{$\frac{\text { Isotopic }}{U(\mathrm{~g} / \mathrm{l})}$} & \multirow{2}{*}{$\frac{\text { Dilution }}{\mathrm{Pu}(\mathrm{g} / \mathrm{\ell})}$} \\
\hline & & & $\mathrm{U}(\mathrm{g} / \mathrm{\ell})$ & $\underline{\mathrm{Pu}(\mathrm{g} / \ell)}$ & & \\
\hline \multirow[t]{12}{*}{5187} & Center & 3.00 & 58.0 & & & \\
\hline & & 13.00 & 95.7 & .510 & & \\
\hline & & 24.00 & 57.9 & & & \\
\hline & & 36.00 & 65.7 & & & \\
\hline & & 45.00 & 61.5 & & & \\
\hline & & 55.00 & 57.3 & & & \\
\hline & & 65.00 & 91.4 & & & \\
\hline & & 75.00 & 61.2 & & & \\
\hline & & 85.00 & 63.2 & .330 & & \\
\hline & Middle & 24.00 & & .477 & & \\
\hline & Outside & 45.00 & 76.1 & .396 & & \\
\hline & & 55.00 & 97.2 & & & \\
\hline \multirow[t]{10}{*}{5185} & Outside & 33.50 & & .400 & & \\
\hline & & 64.50 & & .384 & & \\
\hline & Middle & 12.50 & & .256 & & \\
\hline & & 37.50 & & .319 & & \\
\hline & Center & 2.50 & & .303 & & \\
\hline & & 38.00 & & .264 & & \\
\hline & "Middle & 38.00 & & & 53.38 & .3142 \\
\hline & & 44.25 & & & 51.97 & .3025 \\
\hline & & 55.00 & & & 84.50 & .4987 \\
\hline & & 65.00 & & & 57.19 & .3230 \\
\hline
\end{tabular}


TABLE III

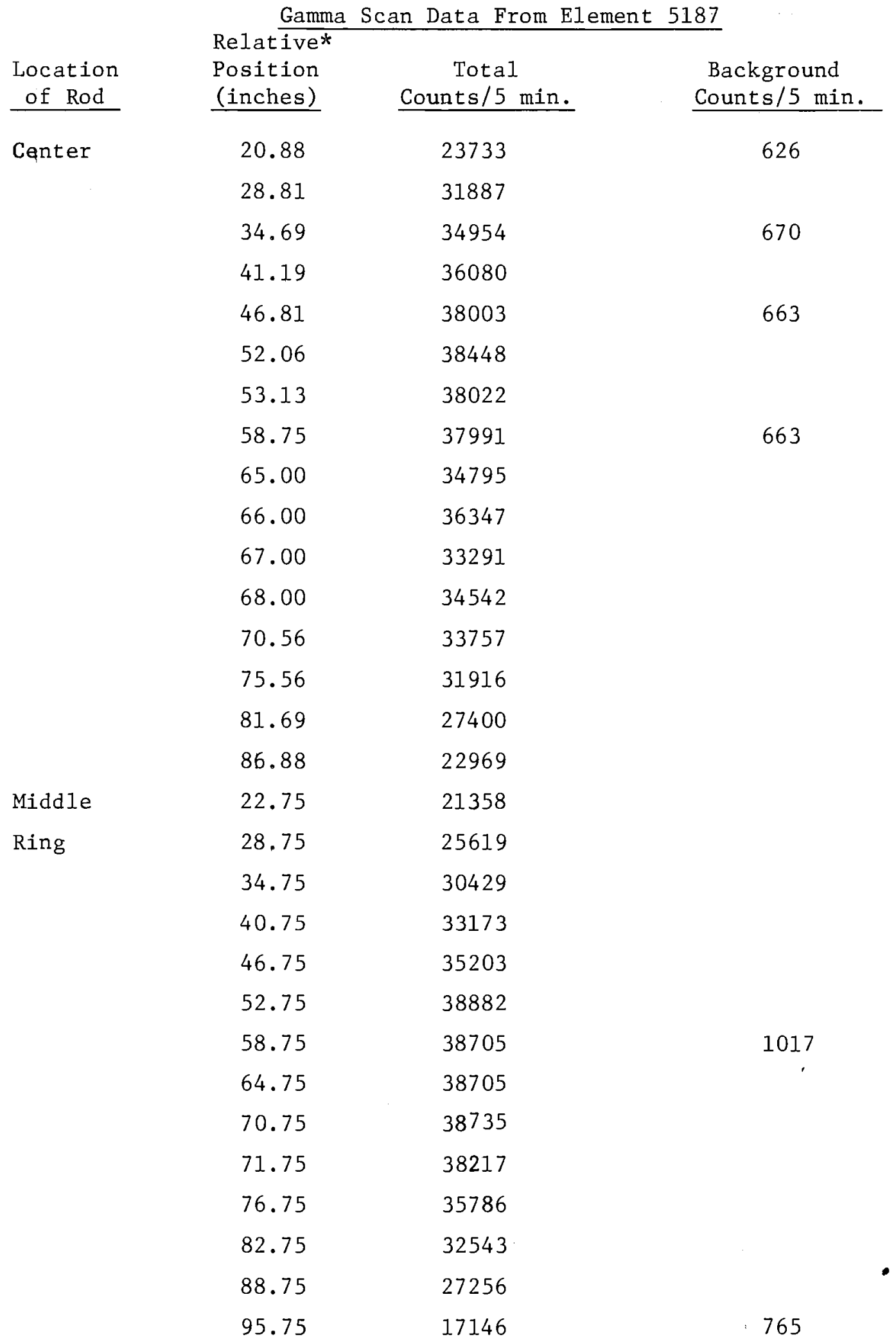

*The fuel begins at a different (but unknown) position for each of the rods. 
TABLE III (contd.)

\begin{tabular}{|c|c|c|c|}
\hline $\begin{array}{l}\text { Location } \\
\text { of Rod } \\
\end{array}$ & $\begin{array}{l}\text { Relative } \\
\text { Position } \\
\text { (inches) } \\
\end{array}$ & $\begin{array}{c}\text { Total } \\
\text { Counts } / 5 \text { min. }\end{array}$ & $\begin{array}{l}\text { Background } \\
\text { Counts } / 5 \text { min. }\end{array}$ \\
\hline Outside & 22.75 & 26104 & \multirow{13}{*}{842} \\
\hline \multirow[t]{12}{*}{ Ring } & 28.75 & 32006 & \\
\hline & 34.75 & 37087 & \\
\hline & 40.75 & 41193 & \\
\hline & 46.75 & 45416 & \\
\hline & 52.75 & 48048 & \\
\hline & 58.75 & 48910 & \\
\hline & 64.75 & 49976 & \\
\hline & 70.75 & 46366 & \\
\hline & 76.75 & 48021 & \\
\hline & 82.75 & 38678 & \\
\hline & 88.75 & 34885 & \\
\hline & 95.75 & 21231 & \\
\hline
\end{tabular}


APPENDIX A 

.... ELENEYT S1E? ANALYSIS OF THE SAMPIE 3.00 INCHES FROM THE TOP OF IHE CENIER ROD

\section{SOTOPIC IN VENTOKY}

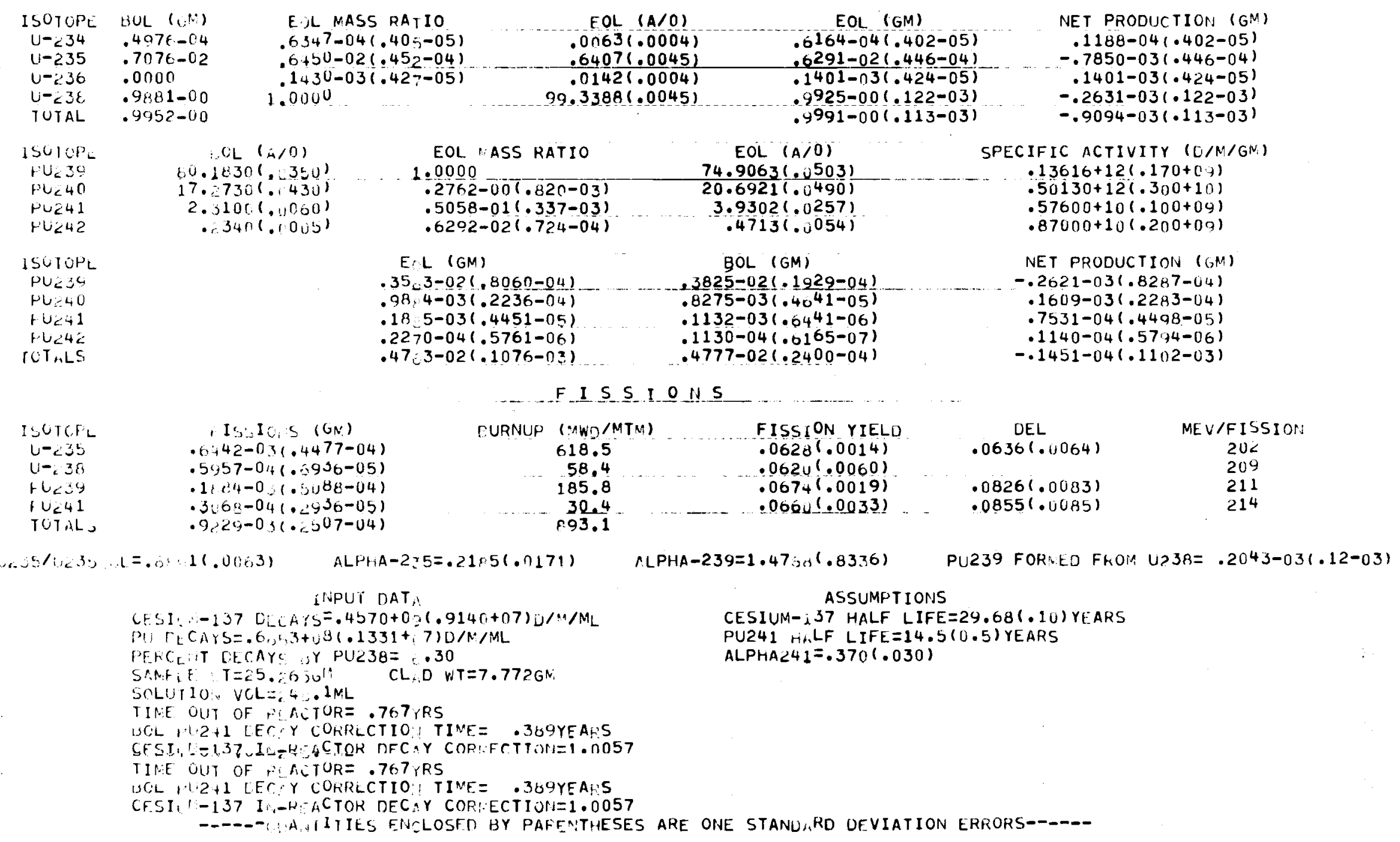




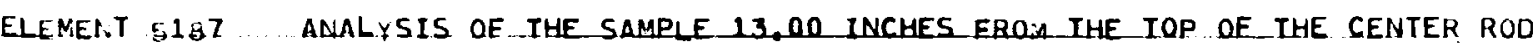

\section{5 O T O P I C I N V E N T O B Y}

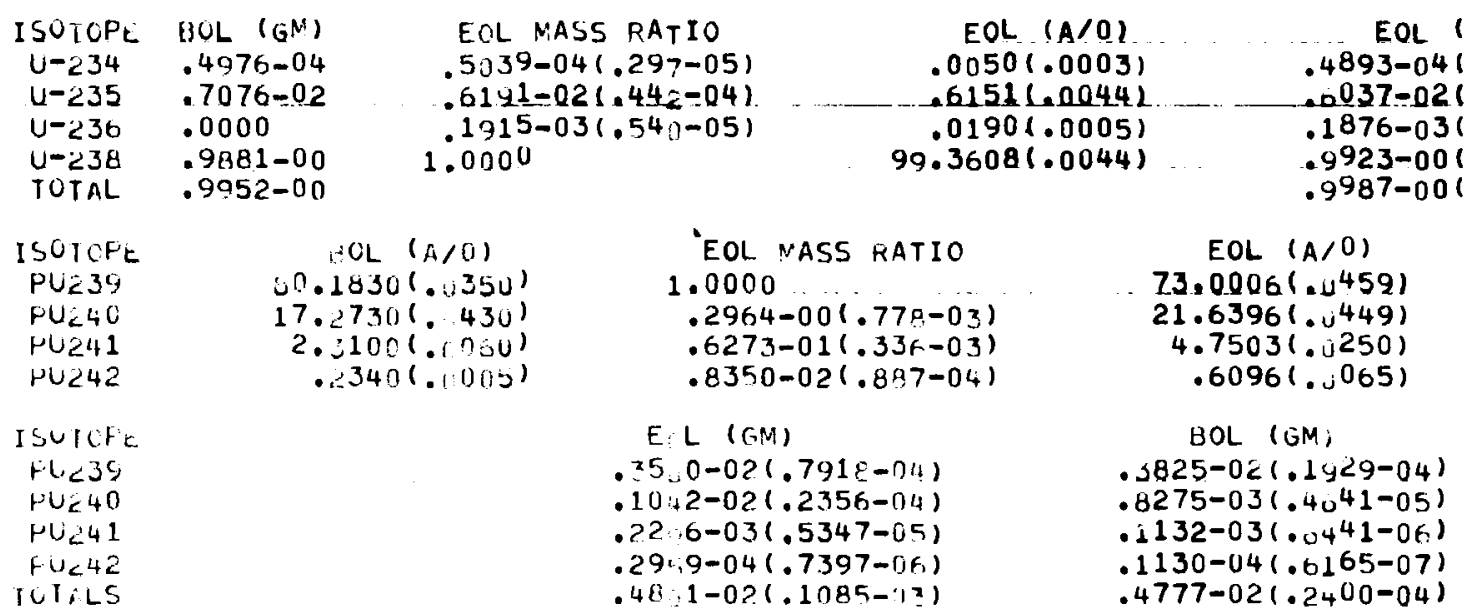

\section{F I S S I 0 if 5}

\begin{tabular}{|c|c|c|c|}
\hline 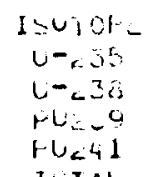 & 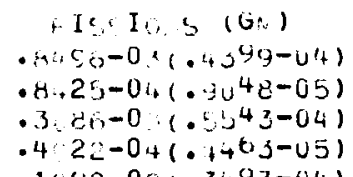 & 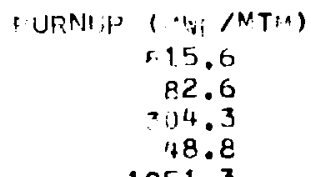 & $\begin{array}{l}\text { FISS ON YIELi } \\
.0620(.0014) \\
.0020(.0060) \\
.0074(.0019) \\
.0004(.0033)\end{array}$ \\
\hline
\end{tabular}

U..35/1.23):RL=.65:c(. U0e1) ALPHA-235=.2227(.n13n)

$A L P H A-239=1 \cdot 2903(.5033)$

NET PRODUCTIOIN (GM)

$-.8277-06(.296-05)$

$-.1039-02(.437-04)$

$.1876-03(.537-05)$

$-.4636-03(.125-03)$

$-.1317-02(.116-03)$

\section{INPUT DAT}

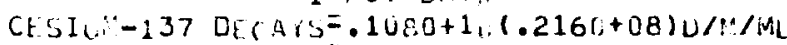

PU CECAYS $=.11 .7+U^{9}(.2314+.7) \mathrm{D} / \mathrm{M} / \mathrm{ML}$

WERCEIT DECAY; UY PUC38= 7,50

SANPLE . $T=37.116 \mathrm{WV} \quad \mathrm{CL} \cdot \mathrm{D} W T=F .963 \mathrm{GM}$

\section{ASSUMPTIONS}

CESIUM- 37 HALF LIFE $=29.68(.10)$ YEARS

PU241 M.LF LIFE $=14.5(0.5)$ YEARS

ALPHAL $41=.370(.031)$

UEL

$.0636(.0004)$

$.0826(.0053)$

$.0855(.0085)$

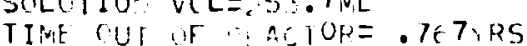

(UI) .1.1241 EECIYY CORRECTIO, TIUE= .3A9YEA:S

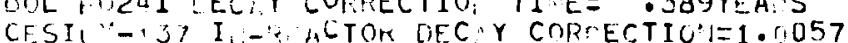

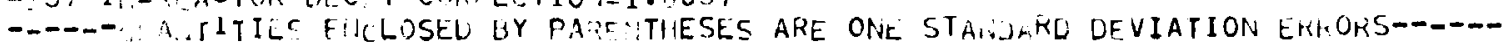


ELEMENT 51L? ANALYSIS OF THE SAMPLE 24,00 INCHES FROM THE TOP OF THE CENTER ROD

\section{ISOTOPIC INVENTORY}

$\begin{array}{ll}\text { ISOTOPE } & \text { TOL }(G M) \\ U-234 & .4976-04 \\ U-235 & .7076-02 \\ U-236 & .0000 \\ \text { U-L3E } & .9081-00 \\ \text { TUTAL } & .9952-00\end{array}$

\begin{tabular}{rr} 
EUL MASS RATIO & EOL $(A / 0)$ \\
$5347-04(.350-05)$ & $.0053(.0003)$ \\
$.5137-02(.48 E-04)$ & $.5801(.0048)$ \\
$.2315-03(.545-05)$ & $.0230(.0005)$ \\
1.0200 & $90.3915(.0049)$ \\
\hline
\end{tabular}

NET PRODUCTION (GM) $.5692-02(.482-04) \quad-.1384-02(.482-04)$ $.2267-03(.541-05) \quad .2267-03(.541-05)$ $.9923-00(.127-03) \quad-.4234-03(.127-03)$ $0984-00(.118-03) \quad-.1585-02(.118-03)$

ISOTCPL PU:39. PU⿺⿻一𠃋十 PU241 PU⿻ 42

ISOTOPE PUद39
PUटि40

rve41

FU 242

TCTALS OL $(A / O)$ EOL MASS RATIO $0.2830(1-350)$

$17.2730(.6430)$
$2.210(.1,060)$
$.2340(.0005)$ 1.0000

$$
\text { EOL }(A>0)
$$$$
-.1585-02(.118-03)
$$
$.3201-00(.902-03)$ $71.0850(.0504)$ $21.0850(.0504)$ $.1090-01(0118-03) \quad .3748(.0083)$

SPECIFIC ACTIVITY $(0 / \mathrm{N} / \mathrm{GM})$

$$
\text { ECL (GM) }
$$

BOL (GM) $.13616+12(.170+09)$ - $50130+12(\cdot 300+10)$ $.57600+10(.100+09)$
$.87000+10(.200+09)$

$\begin{array}{ccc}E_{C} L(G M) & B 0 L(G M) & \text { NET PROOUCTION (GN) } \\ .333_{1}-02(.7501-04) & .3825-02(.1929-04) & -.5137-03(.7745-04) \\ .1064-02(.2409-04) & .8275-03(.4641-05) & .2369-03(.2454-04) \\ .2530-03(.5892-05) & .1132-03(.6441-06) & .1398-03(.5927-05) \\ .3655-04(.9172-06) & .1130-04(.6165-07) & .02524-04(.9193-06) \\ .4665-02(.1055-03) & .4777-02(.2400-04) & -.1117-03(.1082-03)\end{array}$

\begin{tabular}{|c|c|}
\hline $\begin{array}{l}\text { ISUTCPL } \\
U-C 35 \\
U-C 38 \\
\text { PUC39 } \\
\text { FU241 } \\
\text { TOTALS }\end{array}$ & $\begin{array}{c}\text { FIstI(s }\left(G_{i v}\right) \\
.1154-0(.4852-04) \\
.1(96-03(.1151-04) \\
.3595-03(.6567-04) \\
.6795-04(.6039-05) \\
.1691-02(.4560-04)\end{array}$ \\
\hline
\end{tabular}

FIS5:0NS

$\begin{array}{ccc}\text { PURNUP (MWO/MTM) } & \text { FISS ON YIELD } & \text { DEL } \\ 1107.5 & .0626(.0014) & .0636(.0064) \\ 107.5 & .0620(.0060) & \\ 354.4 & .0674(.0019) & .0826(.0083) \\ 67.4 & .0664(.0033) & .0855(.0085)\end{array}$

MEV/FISSION
202
209
211
214

U. $35 / U A 30: L L=.50446 .0068$

ALPHA-2:5 $=.1905(.0101)$

$A L P H A-239=1.30727 .44081$

PUZ39 FORMED FROM U238=.3152-03(.13-03)

$$
\text { INPUT DAT/ }
$$
ASSUMPTIONS

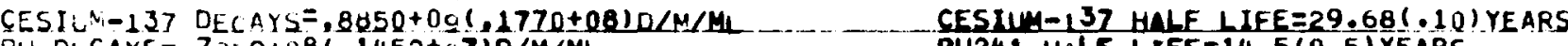

PU DECAYS $=.72 L 9+08(.1452+07) 0 / \mathrm{M} / \mathrm{ML}$

PERCENT DECAYS HY PU238 8.70

SAMPLE $T=25 . K 38 G^{N_{1}} \quad C L, Z O W T=6.807 \mathrm{GM}$

SOLUT ION VOL $=255.0 \mathrm{ML}$

SAMPLE :I $=23$, $R 3$ SOGN

$C L$ ID $W I=6.807 \mathrm{GM}$

SOLUT ION VOL $=255.0 \mathrm{ML}$

TIME OUT OF PEACTOR= .847YRS

BOL PU241 CECAY CORRECTION TIMEE - 389YEARS

$$
\text { -----OUANTITIES ENCLOSED BY PARENTHESES ARE ONE STAMDARD DEVIATION ERRORS---- }
$$


ELEMENT \$137 ANALYSIS OF THE SAMPLE 36.00 INCHES EIUW IHE IOP OF THE CENTER ROO

\title{
I SOOTOP I C I NVENTOKY
}

$\begin{array}{lll}\text { ISUTOPE } & \text { HCL (CN) } & \text { EUL MASS PATIO } \\ U-234 & .4376-04 & .5+U 2-04(.28=-05) \\ U-C 35 & .7076-02 & .5539-0<(.46-04) \\ U-C 3 E & .0000 & .2+42-03(.101-04) \\ U-238 & .9881-00 & 1.0000 \\ 101 A L & .9352-00 & \end{array}$

EOL $(A / 0)$
$.0054(.0003)$
$.5606(.0046)$
$.0248(.0010)$
$90.4093(.0047)$

EOL (GM)

$.3242-04(.284-05)$

$.0495-02(.457-04)$

$.2439-03(.103-04)$

$. .9916-00(.140-03)$
ISUTOFE OU $(A, 0)$

U $<\leq 39$

Pli 40

PUतil 1

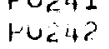

ISUICF

$f v_{c}=9$

Fic 40

Fi: $<<4$

ICTULS

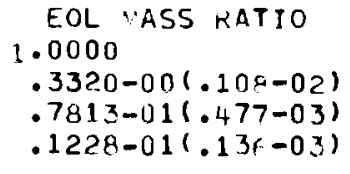

EOL VASS RATIO

1.0000

$.3320-00(.108-02)$

$.7813-01(.477-03)$

$.1228-01(.13 f-0.3)$

E- L (GM)

$. \times 6 \geq 5-0 ?(.8243-34)$

$.1212-02(.2745-1) 4)$

$.2970-03(.6986-05)$

$45: 0-04(.114 n-05)$

$519-02(.1174-03)$
EUL $(A, 0)$

$70.1587(.1592)$

$23.2934(.590)$

$5.6802(.4337)$

$.8618(.095)$

BOL (GM)

$.3825-02(.1,29-04)$

$.0275-03(.4041-05)$

$.1132-03(.0441-06)$

$.1130-04(.0465-07)$

$.4777-02(.24,00-04)$

F I S S I 0 II $S$

Isvictis

$u-2$
-35

$4-23 t$

pes?

PUCH
TUTAL

\begin{abstract}
I5) I $5(0)$
\end{abstract}
$.1 \div 32-02(\cdot+0) \div 7-04)$

$.1-49-03(.1342-04)$

- 5 ग-0 $0(0.7140-0+1)$

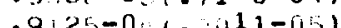

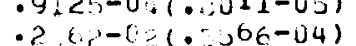

EURNIP (NWI:MTA)

$1 ? 78.8$

132.3

$\because 96.5$

1093.0

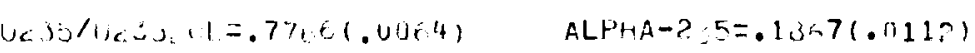

$: L P H I n-239=1.3767(.3511)$

FISS: UN YIEL:

$.0020(.0014)$

$.0620(.0060)$

$.0674(.0019)$

.066 r.00331
SPECIFIC ACTIVITY (D/MOGH)

$.13616+12(.170+04)$

$.50130+12(.300+10)$

$.57600+10(.1110+0 i j)$

$.87000+10(.200+09)$

NE 5 PRODUCTION $\left(G M_{1}\right)$

$-.1906-03(.8465-04)$

$.3 .343-03(.27 .44-(14)$

$.1838-03(.7015-65)$

$.3990-04(.1142-05)$

$.4115-03(.1109-03)$

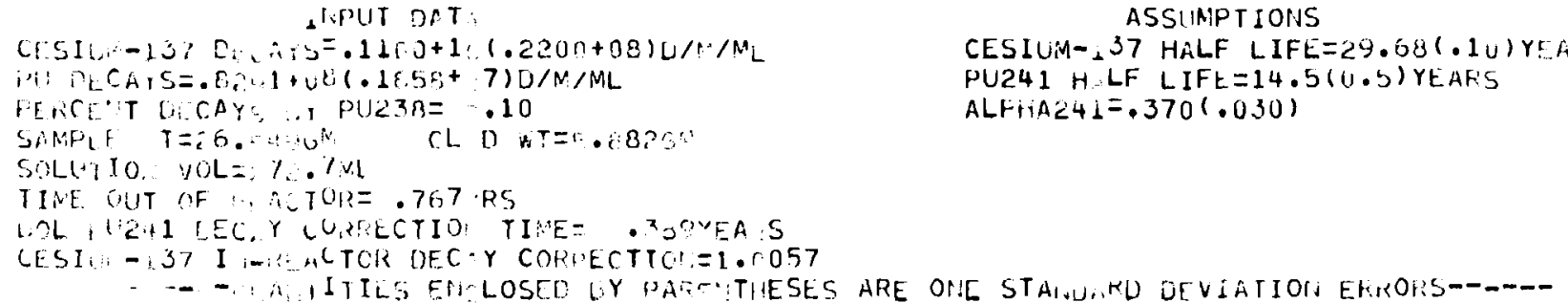


ELEMEUT E187 ALALYSIS OF THE SAMPIE 45.00 INCHES FROM THE TOP OF THE CENTER ROD

I SOTOPIC INVENTOHY

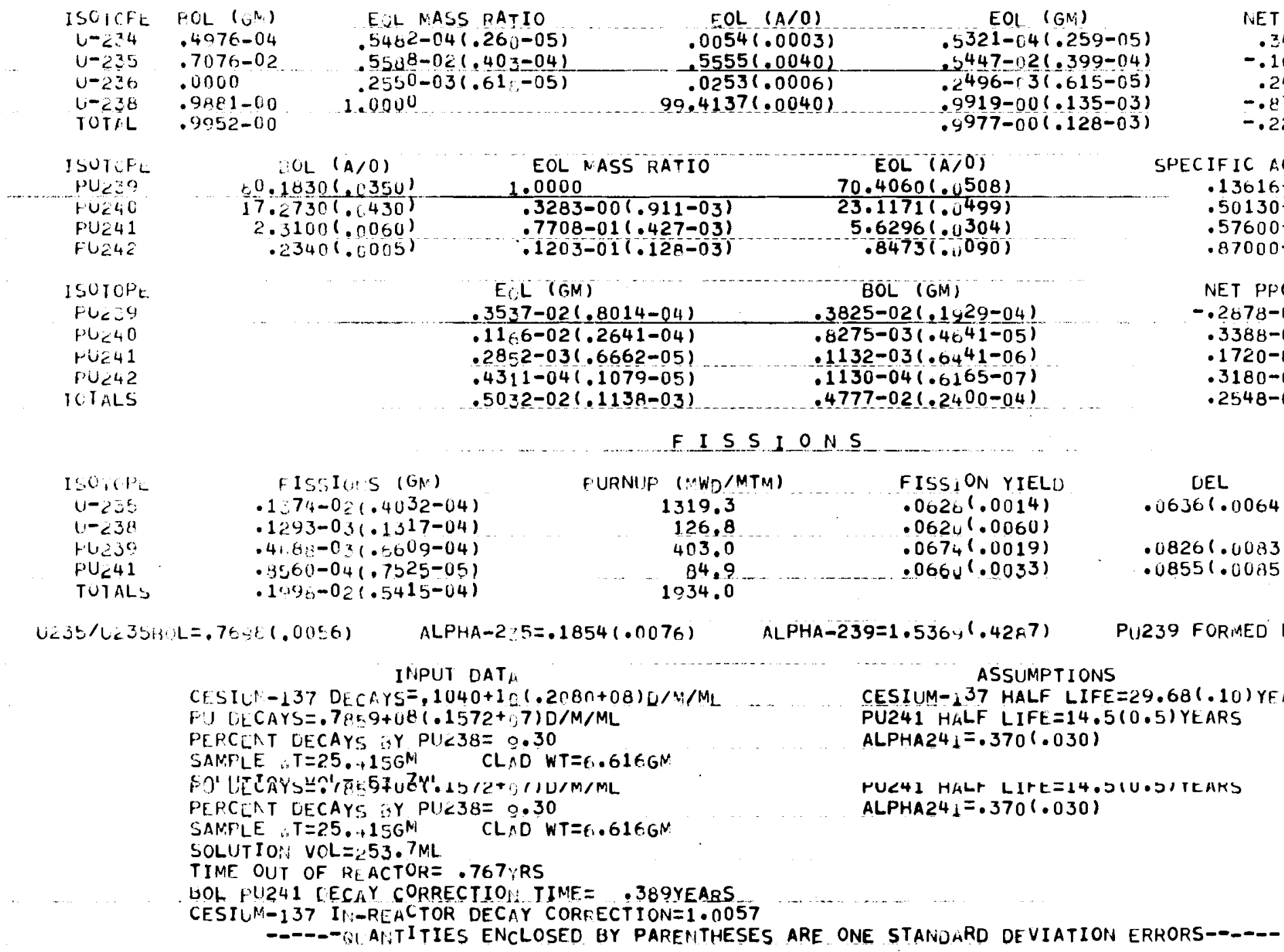


ELEMEIT :IH7 ANALYSIS OF THE SAMPLE 55.00 INCHES FROM IHE TOP OF THE GENTER ROD

I S O T O P I C I N V E N.TOK

U. $034 \quad .4976-04$

$\begin{array}{ll}0-236 & .9881-C 0\end{array}$
EOL (GM)

$.5620-02(.495-04)$

$.2345-03(.534-05)$

$.9917-00(.139-03)$

$.9977-00(.130-03)$
$.5014-04(.324-05)$

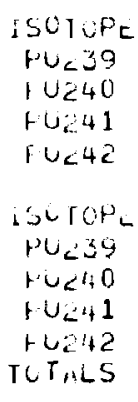

ISUTOFE

$0-\angle 35 \quad .7076-02$

$\mathrm{L}=236 \quad .0000$

$.9952-00$

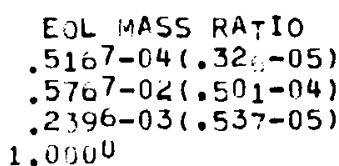

$$
\begin{array}{r}
.01(1 . / 6) \\
.0 .1830(.354) \\
17.2730(.430) \\
2.100(.000) \\
.234(.00150)
\end{array}
$$

$$
\begin{aligned}
& \text { EOL *ASS }: A T 10 \\
& 1.0000 \\
& .3195-00(.132-02) \\
& .7431-01(.531-03) \\
& .1113-01(.125-03)
\end{aligned}
$$

$E$ L (GM)

.36:5-02(.8301-(14)

$.1173-02(.266 n-04)$

$.28: 1-03(.6772-05)$

$.41 .1-04(.1044-05)$

.51 .3-02(.1167-03)

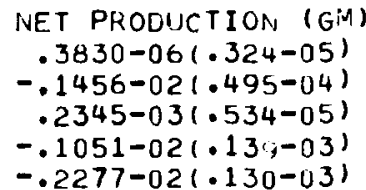

EOL $(A / 0)$

$71.0309(.4727)$

$22.6963(.1,732)$

$5.4759(.379)$

$.7910(.088)$

BOL (GM)

. 3825-02(. $+429-04)$

$.8275-03(.4041-05)$

$.1132-03(.6441-06)$

$.1130-04(.5105-07)$

$.4777-02(.2400-04)$

FI SSI.ONS

Loulde

L $-2.5 \mathrm{~s}$

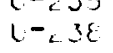

ruisa

PUC 41

IUTHLS

f ISEISS (0)

- $1,17-0<(0+78-04)$

- $1,4<-03(0128-04)$

$.4743-0 \%(.7020-04)$

. $11.20-0,\left(0.71 v_{0}-05\right)$

$.146-0 ;(.5119-04)$

IURNIIP $(\because$ NOMT:1)

1168.4
121.8
167.7
79.8

1168.4

1467.7

18.37 .7

\begin{abstract}
FISS 1 ON YIEL-
$.0628(.0014)$

. Dózu $(.0060)$

$.0674(.0019)$

.066. (.0033)
\end{abstract}

SPECIFIC ACTIVITY (D/A/GM)

$.13016+12(.170+09)$

. $50130+12(.300+10)$

$.57000+10\left(.1_{13} 0+0.3\right)$

$.87000+10(.200+0 y)$

NE.T PRODUCTIOH (GP)

$-.1700-03(.8522-04)$

$.3452-03(.27) 0-04)$

$.1709-03(.68173-05)$

$.2991-04(.1046-05)$

$.3761-03(.1191-03)$

\section{LEL}

$.0826(.0083)$

$.0855(.0085)$

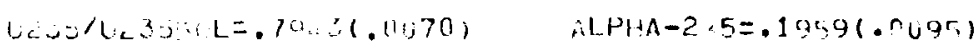

ALPHA $-239=1.3<U \rightarrow(.3657)$

PIÉ39 FORIMED FROM USSH $=.9303-03(.14-03)$

ASSUMPTIONS

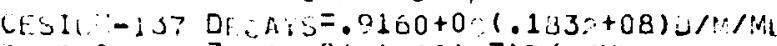

P1: $D_{t} \mathrm{CA}, S=.7=\hat{2}+\mathrm{U}^{\mathrm{R}}(.1460+7) \mathrm{D} / \mathrm{MI} / \mathrm{ML}$

HEFICETT LECAY Y FNICZB= .00

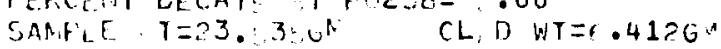

SOLUYIO: VCL $=$ AORL

TINF CU: OF, ALTCRE .767:RS

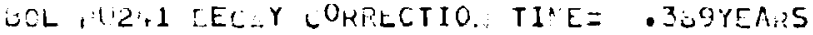

CESIU:-37 I . W HCTOK OFC, Y COPRECTIO:=1. 1:057

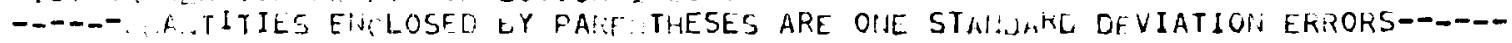

CESILIM- -37 HALF LIFE $=29.601 .10)$ YFARS

H.LF LIFE $=14.5(0.5)$ YEAPS

ALPHAC41 $=.370(.030)$ 
ELEMER.T E1.77 ANALYSIS OF IHE SAMPLE 65.00 INCHES FROM THE TOP OF THE CENTER ROD

\section{ISOTOPIC INVENTOKY}

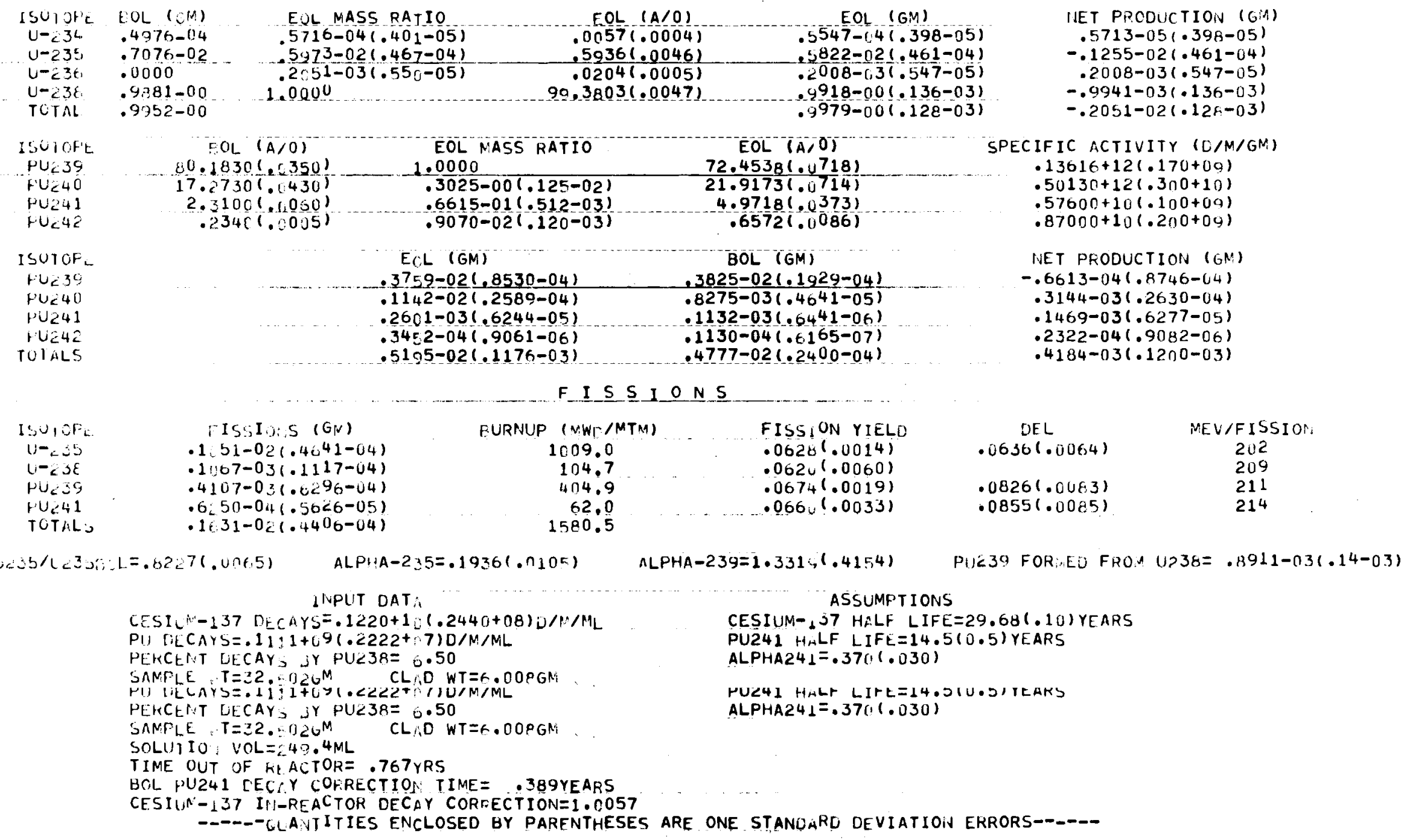


ELEMET:T $: 107$ ANALYSIS OF THE SAMPLE 75.00 IUCHES FGOE. IHE TOP OF THE CENTER ROD I 5 O T O P P I C I NVE N TO R Y

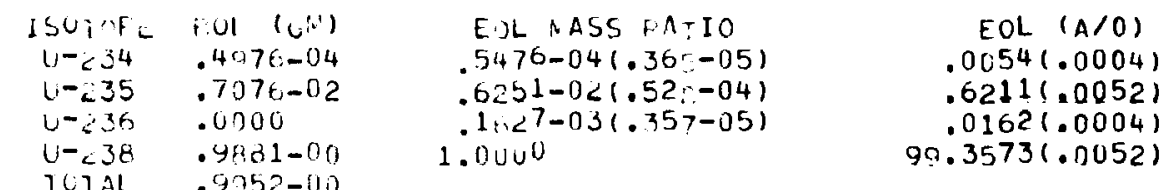

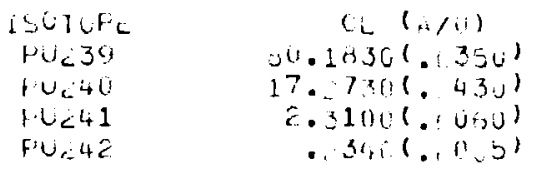

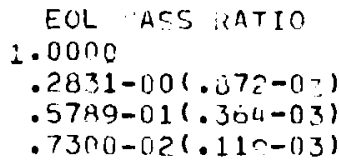

\section{E. $L(G N)$}

ISUTOF

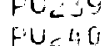

$10<+1$

TUTALS

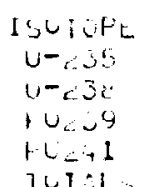

$u-636$

$0-132$

$r<1$
$.37 .1-02(.6375-04)$

$.10 \cdot 2-02(.2380-04)$

- 22 1-03(.527)-(15)

$.501 .4-0 \geq(.1131-0 \geq)$
$.22,1-03(.5270-05)$

$$
\text { 1.0 } 000
$$

$90.3573(.0052)$

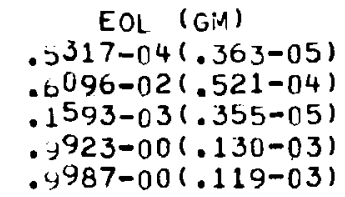

EOL $(A, 0)$

$74.0493(.527)$

$20.9634(. .514)$

$4.4468(.0<74)$

$.5406(.0088)$

BOL ( $\mathrm{GM})$

$.3825-0<(.1029-04)$

$.6275-03(.4041-05)$

$.1132-03(0.041-06)$

$1130-04(00105-07)$

$.1130-04(.0105-07)$

F I 5 S I 0 in $S$

\begin{abstract}
IS:IUi: $=(G . v)$

\begin{tabular}{|c|c|}
\hline \multirow{5}{*}{ UQNIIP } & $\left.(\cdots, / N T \cdot)^{\prime}\right)$ \\
\hline & 736.9 \\
\hline & 951.4 \\
\hline & 42.8 \\
\hline & .48 .9 \\
\hline
\end{tabular}

$\cdot 1,10-0 \div(.5222-(14)$

- bion-0ik $73-45$

- $1-36-0-(2,02-04)$

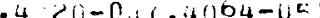

$.1 .00-0 ?(0.977-44)$
\end{abstract}

FISSIUN YIELH
$.002(.0014)$
$.002(.0060)$
$.0674(.0019)$
$.0000(.0033)$

$1 \times 48.9$
NET PRODUCTION (GiA)

.3408-05(.363-05)

$-.9805-03(.521-04)$

$.1593-03(.355-65)$

$-.4907-03(.13(0-03)$
$-.1314-02(.119-03)$
SPECIFIC ACTIVITY (L:M/GM)

$.13016+12(.170+04)$

$.50130+12(.300+1)$

$.57600+10(.100+00)$ $.87000+1(1.200+00)$

NET PFOOUCTION (i,

$-.1245-03(.8504-64)$

$.2246-03(.2425-44)$

$.1109-03(.53109-4:)$

$.1605-04(.7657-65)$

$.2270-03(.1156-0.3)$

ASSUMPTIONS

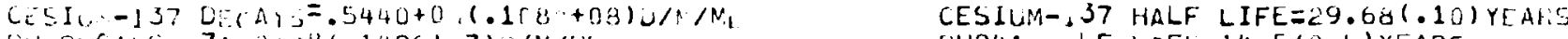

Fi: C:CAYS=.71: $3+J 4(.1426+7) \cup / M / N L$

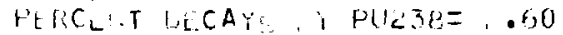

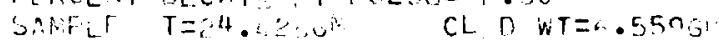

PU241 LF LIFE $=14.5(0.5)$ YEARS

ALPHAC4 $=.370(.030)$

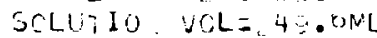

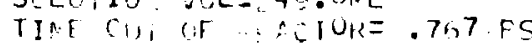

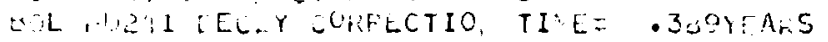

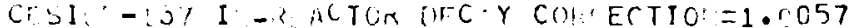

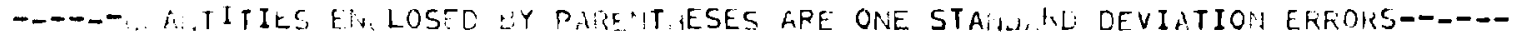


.... ELEME:T G1OZ ANALYSIS OE IHE SAMPLE 85.00 INCHES FROLL.THE TOP OF THE CENTER RCD I.SOTOP I C INVENTO Y

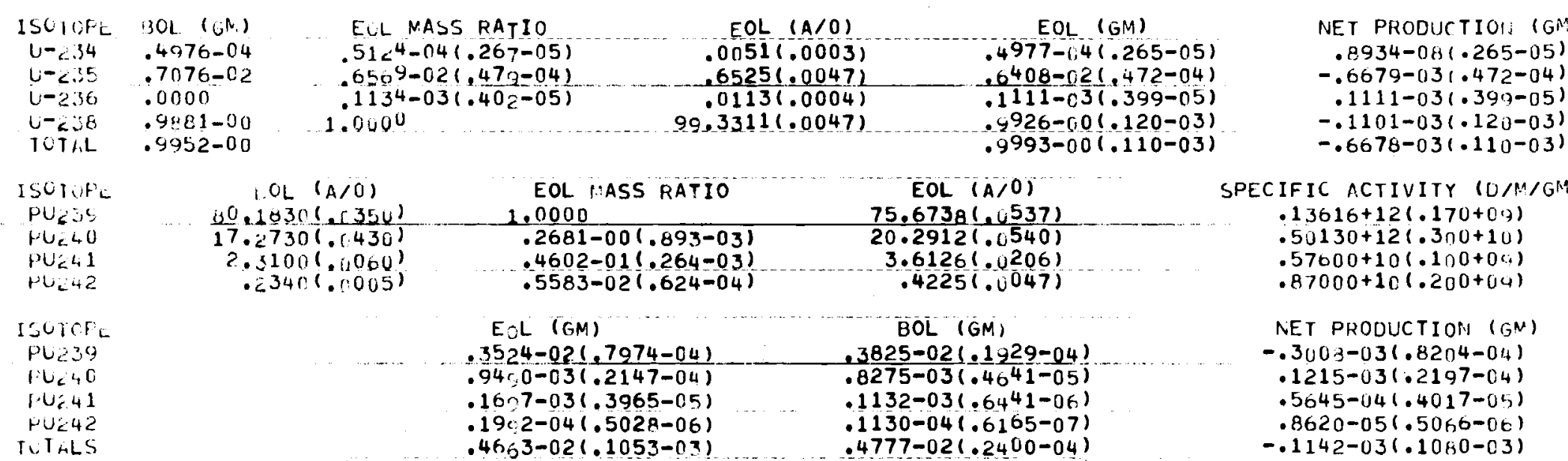

F I S S I O NS

\begin{tabular}{|c|c|c|c|c|c|}
\hline 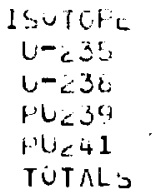 & $\begin{array}{r}(1 S \mathrm{I})(\mathrm{S}(\mathrm{G}) \\
.5564-03(.4740-04) \\
.5 .123-04(.5312-05) \\
.1513-03(.5104-04) \\
.232(1-04(.2323-05) \\
.7111-03(.2127-04)\end{array}$ & $\begin{array}{l}\text { FURNUP (NWT/NTM) } \\
534.1 \\
49.3 \\
149.2 \\
23.0 \\
755.5\end{array}$ & $\begin{array}{l}\text { FISSION YIELE } \\
.0623(.0014) \\
.0620(.0060) \\
.0674(.0019) \\
.0660(.0033)\end{array}$ & $\begin{array}{c}\text { UEL } \\
.0636(.0054) \\
.0826(.0083) \\
.0855(.0685)\end{array}$ & $\begin{array}{c}\text { MEV/FISSIOH } \\
202 \\
209 \\
211 \\
214\end{array}$ \\
\hline
\end{tabular}

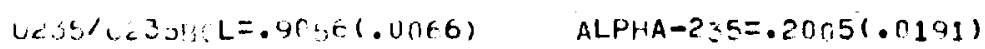

ALPHA-239=1.3065(*****)

P(1239 FORIED FROM UP3R $=.6008-04(.12-0.3)$

\section{INPUT DATA}

CESIUI- 37 DECAYS $=.4310+0 C(.862 C+07) 0 / \mathrm{M} / \mathrm{ML}$

PU DECAYS $=.7143+08(.1440+C 7) \mathrm{D} / \mathrm{M} / \mathrm{ML}$

PEKCEINT LECAYS YY PLIZ38 $=5.90$

SAMFLE, T $=25,1620 \mathrm{M}$

SOLUT IO $V \mathrm{OL}=51.9 \mathrm{ML}$

TIME OUT OF RFACTOR= .767YRS

EOL FU2"1 DECAY CORRECTIO:A TIME= .389YEARS

CESIUVi-137 INI-REACTOR DECAY CORRECTION=1.0057

-

\section{ASSUMPTIONS}

CESIUM- 37 HALF LIFE $=29.681 .10$ YYEARS

ALPHA241 $=.370(.030)$
PU241 HHLF LIFE $=14.5(0.5)$ YEARS 


\section{ELEMEKT '.LIS7 ANALYSIS OF THE SAMPLE 3.00. INCHES EROM THE TOP OF. THE MIDDLE ROD}

\section{5 O T O P I C I NVENTO I Y}

$\begin{array}{lllr}\text { ISUTSPE } & \text { BOL (GM) } & \text { EOL NASS BATIO } & \text { EOL (A/O) } \\ U-C 34 & .4976-04 & .5707-04(.377-05) & .0057(.0004) \\ U-235 & .7076-02 & .6052-02(.39-04) & .6607(.0039) \\ U-C 30 & .0000 & .1109-03(.380-05) & .0110(.0004) \\ U-23 E & .9981-00 & 1.00 U U & 99.3226(.0040)\end{array}$

EOL $(G M)$
$.598-04(.374-05)$
$.0485-02(.393-04)$
$.1086-03(.377-05)$
$.9920-00(.126-03)$
$.9987-00(.119-03)$

NET PRODUCTION (GM)

$.622(1-05(\cdot 374-05)$

$.1086-03(.377-05)$

$-.7914-03(.12(10-03)$

$-.1275-02(.113-03)$

TUT TAL

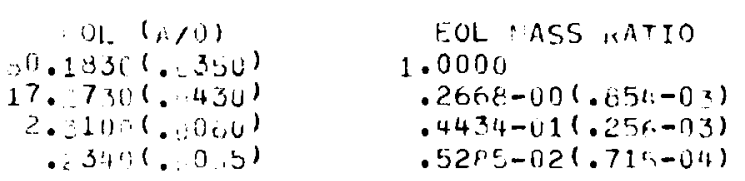

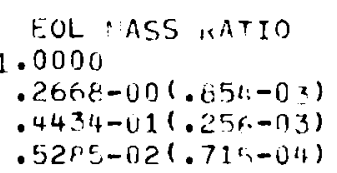

EOL : ASS KATIO

$.2668-00(.65 t:-03)$ $.4434-01(.254-03)$ $.52 f s-02(.71(i-04)$

$$
\text { E! L }(E M)
$$

$.38 .3-02(.6501-04)$

. $17.9-03(.4112-05)$

$.2035-04(.5362-014)$
$.5(118-02(.1132-02)$

\section{F I S S I 01,5}

\section{lSUAOFL \\ u-. 3is \\ $i-c: 3 E$ \\ rucis \\ Fic 41 \\ IUTAL}

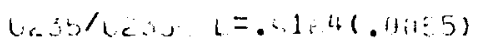

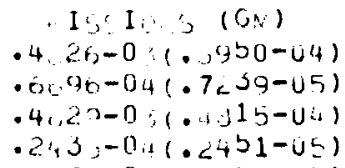

IIURNIT ( 'Wh /MT:)

463.3

65.7

1.56 .4

24.1
1009.5

IURNIT $(\cdots, / M T$
463.3
65.7
156.4
24.1
1009.5

$.20: 9-0 ?(.2305-011)$

EOL $(A / 0)$
$75.8754(.517)$
$20.2428(.519)$
$3.4808(.0199)$
$.4010(.054)$
BOL (EM)
$3825-0<(.1 .729-04)$
$3275-03(.4641-05)$
$1132-03(.0441-06)$
$1130-04(.0105-07)$
$4777-02(.2+00-04)$

FISS, (NN YIELIS
$.002(1.0014)$
$.0024(.0060)$
$.0674(.0019)$
$.0004(.0033)$

SPECIFIC ACTIVITY $(U / M / G M)$ $.13016+12(.170+0.2)$ $.501 .30+12(.3 ! 10+1 !))$ $.57600+10(.100+0(4)$ $.87000+16(.2, j 0+0: s)$

INET PRODLCTIOH $(0, M)$ $-.225:-04(.0815-04)$ $.1913-1) 3(.23,1-114)$ $.6270-04(.4162-05)$ $.9046-05(.536 ; 8-6,6)$ $.2404-03(.11+, 4-03)$

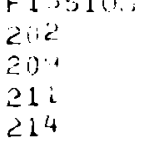

$.0706(.01877)$ $.0799(.060 .50)$
ALFHA-239= .62U-.(.3211)
P:1239 FOR ,L: FHOR U. $38=.7275-(13(.13-13)$
ASSUMFTIONS

CESIUVI-137 HALF LIFE $=29.068(.1 U)$ YFARS

PU241,.LF LIFE $=14.5(\mathrm{U} .5)$ YEARS

PU24 1, LF LIFE $=14.5(4)$
ALPHIA $241=.370(.030)$

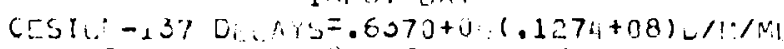

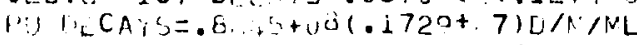

HEKC!NT DECAY Y PLC38= .50

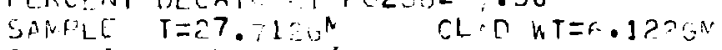

SOLHIO VOL $=5 \%, 3 \mathrm{NL}$

TINE OU: OF ACTORE .712:RS

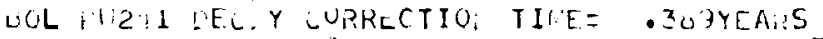

CFSI - 37 I - ACTOK LFC Y COPECTIOH=1.0057

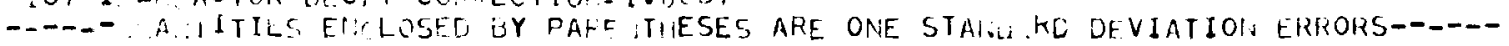


ELEMENI L18? ANALYSIS OF THE SAMPLE 13.00 INCHES FROR THE TOP OF THE MIDDLE ROD

\title{
I 5 O TOP I C I NVENTORY
}

\begin{tabular}{|c|c|c|c|c|c|}
\hline $\begin{array}{l}1 S O, J F t \\
U-234 \\
U-235 \\
U-236 \\
U=36 \\
1 U T 3 L\end{array}$ & $\begin{array}{l}\text { EOL }\left(\mathrm{UN}^{N_{i}}\right) \\
.4976-04 \\
.7076-02 \\
.0000 \\
.9881-00 \\
.9952-00\end{array}$ & $\begin{array}{l}\text { EOL MASS RATIO } \\
.5121-04(.237-05) \\
.6291-02(.344=04) \\
.1416-03(.476-05) \\
1.0000\end{array}$ & $\begin{array}{r}\text { EOL }(A / 0) \\
.0051(.0002) \\
.6250(.0034) \\
.0141(.0005) \\
99.3558(.0034)\end{array}$ & $\begin{array}{c}E O L(G M) \\
.4969-C 4(.236-05) \\
.61 \frac{13}{1}-02(.339-04) \\
.1386-03(.473-05) \\
.9916-00(.131-03) \\
.9981-00(.126-03)\end{array}$ & $\begin{array}{l}\text { NET PRODUCTION (GA) } \\
-.7005-07(.236-05) \\
-.0453-03(.33(1-04) \\
.1386-03(.473-05) \\
-.1116-02(.131-03) \\
-.1925-02(.126-03)\end{array}$ \\
\hline
\end{tabular}

$.9952-00$

LOL $(A / O)$

EOL PASS RATIO

EOL $(A>0)$

SPECIFIC ACTIVITY (O/M/GIU) $\frac{0.1830(.0350)}{17.2730(.0430)}$ $17.2730(.0430)$
$2.3100(.0060)$ 1.0000

$74.1571(.0483$

$.13616+12(.170+09)$

$.50130+12(.350+10)$

$57000+10(.100+09)$

$.2340(.0005)$

$.5366-01(.287-03) \quad 4.1170(.0218)$

$4.1170(.0218)$
$.5073(.0057)$

$.87000+10(.200+09)$

$E L^{-}(\mathrm{GM})$

BÖL (GM)

ISUT $2 P_{L}$

PU: 39

F $v_{c}+0$

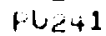

Pप: 4242

TOTALS

$.38 \div 4-02(.8652-04)$

$.1099-02(.2485-04)$

$825-02(.19209-04)$

NET PROOUCTION (GM)

-.1314-05(.8864-04)

$.2712-03(.2528-04)$

$.2141-03(.4983-05) \quad .1132-03(.0441-06)$

$.2649-04(.6706-06)$

$1132-03(.0441-06)$

$.1009-03(.5024-05)$

$.1518-04(.6735-06)$

$.51,3-02(.1167-03)$

$.4777-02(.2400-04)$

$.3860-031.1191-03)$

FISSIONS

$15 O T C P L$

$U-<35$

$u<38$
$P U 23 c$

P.

IEURNUP

\begin{abstract}
FIScIO 5 (Givi)
-8154-0,3(.3426-04)

$.902(-04(\cdot 1000-04)$

$.5056-0>(.5299-04)$

$.4 .87-04(.3777-05)$

$.1540-02(.1+205-04)$
\end{abstract}

(NWONTM)

773.1

FISSION YIELO
$.0620(.0014)$

DEL

$.0609(.0061)$

MEV/FISSIOI:

96,4
587.2

$.0624(.0060)$

$40.5 \quad .0674(.0019)$

$.0766(.0077)$

202
209

$1407: 3$

$.0799(.0080)$

211

TUTALS

ALPHA $-2: 5=.1736(.0101)$

ALPHA-239= $.7136(.2574)$

PLj239 FORAED FROM U>3 $i=.1022-0 \%(.13-03)$

INPUT DAT/A

CESILI:- 137 DELAYS $=, 9900+09(.1980+08) D / M / M L$

PIJ CLCAYS $=.94,4+08(.1889+07) 0 / \mathrm{M} / \mathrm{ML}$

PERCENT DECAYS BY PU23B= $\div .40$

SAMPLE $T=-8.2476 \mathrm{M} \quad \mathrm{CL}: D W T=5.736 \mathrm{GM}$

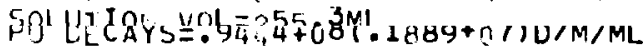

PERCLNT DECAYS IIY PU238 $=7.40$

SAMPLE T=C.8. $8.47 G M \quad C L: D W T=5.736 \mathrm{GM}$

ASSUMIPT IONS

CESIUM $=137$ HALF LIFE $=29.68(.10)$ YFARS

PU241 HALF LIFE $=14.5(0.5)$ YEARS

ALPHA241 $=.370(.030)$

PU24 I HALF LITE $=14.01 U \cdot O I T E A K S$

SOLUTIOI. VOL $=255.3 \mathrm{ML}$

ALPHA2 $41=.370(.030)$

TIME OUI OF REACTOR= .712YRS

BOL FU241 CECSY CORRECTIOU IIME = .389YEAES

CESIUM-137 IN-REACTOR DECAY CORNECT ION=1.0057

--.-OUANTITIES ENCLOSED BY PARENTHESES ARE ONE STANDARU DEVIATION ERRORS---.-- 


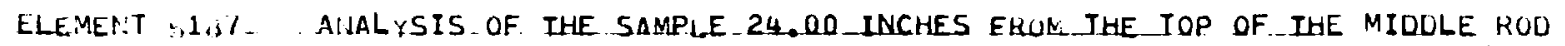

\section{IS OTOPI C I NVENTOKY}

$\begin{array}{ll}\text { ISUTCFE } & \text { HOL }(\mathrm{G} \text { (9) } \\ U-C 34 & .4976-04 \\ U-\angle 35 & .7076-02 \\ U-236 & .0000 \\ U-C 3 E & .9801-110 \\ \text { TUTAL } & .9752-00\end{array}$

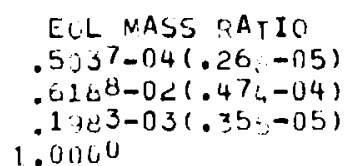

$E O L(A / 0)$

$.0050(.0003)$

$.6148(.0047)$

$0197(.0004)$

$90.3605(.0047)$ sorote UI $(i / 0)$

$\mathrm{PUCS}_{<3}$

$+U_{<} 40$

$P U_{c+1}$

एᄂ 242

isU1CH'

小⿺廴⿻肀二𠃋

$10 x^{2}=0$

$\mathrm{F}-\mathrm{U}_{\mathrm{C}+\mathrm{H}} \mathrm{1}$

$+6<42$

TuTal.s

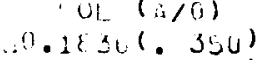

$$
\begin{aligned}
& \begin{array}{c}
0.1830(\cdot 354) \\
17.2730(.400)
\end{array} \\
& \text { 2. } 1 \text { ar (.: } 0 \text { osu } \\
& \text {. } 34 \text { ir. (. Os) }
\end{aligned}
$$

$$
\begin{aligned}
& \text { EOL ASS I.ATIO } \\
& 1.0000 \\
& .3143-00(.135-02) \\
& .0645-01(.350-03) \\
& .9435-02(.112-03)
\end{aligned}
$$

E. L $(C \cdot M)$

$.36 .4-02(.8323-134)$

$.11 .7-02(.2624-014)$

$.2510-03(.5975-05)$

$.25(1-04(.9076-06)$

$.5110-02(.1157-(1, x)$
EOL (GM)

$.4886-04(.264-05)$

$.2028-02(.468-04)$

$.1940-03(.353-05)$

$.9913-00(.139-03)$

$.977-00(.131-03)$
I.ET PRODUCTIUA, (GM) $-.0012-06(.264-05)$ $-.1048-02(.463-04)$ $1940-03(.353-03)$ $-.1474-02(.13 y-03)$ $-.2330-02(.131-03)$

FISSIONS

ris

TCTIL

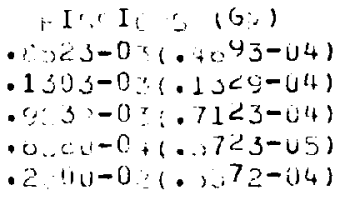

EOL $(A / 0)$

$71.8140(.4733)$

$22.5712(.0755)$

$4.9372(.0291)$

$.6776(.1 .084)$

LOL (GM)

.3825-02(.1 $220-04)$

$.8275-03\left(.4 u^{4} 1-05\right)$

$.1132-03(.0 .41-06)$

$.1130-04(.0165-07)$

$.4777-02(.2400-04)$
SPECIFIC $/ C T I V I T Y \quad([/ N / G M)$

$.13616+12(.170+6, i)$ $.50130+12(.3,30+10)$ $.5760)+16(.1,0+0)$ $.87000+1 ;(.200+0 y)$

IIET PrODLCTISIN $(i, \cdots)$ $-.1006-03(.05: 4-0.4)$ $.3291-03\left(.2 r, r^{4}-04\right)$ $1403-03(.60104-1 ;)$ $.2371-04(.91) .7-\ldots 0)$ $.3330-03(.1151-0.3)$

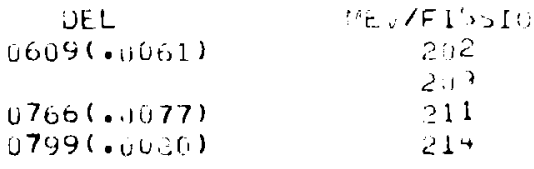

P.,239 FORLL FRO" $1034=.1354-(12(.14-03)$

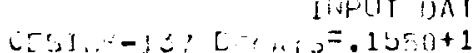

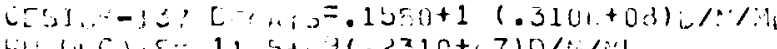

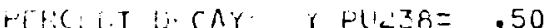

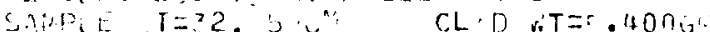

SOLLI:O HOL $=4,2 \mathrm{KL}$

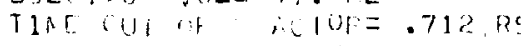

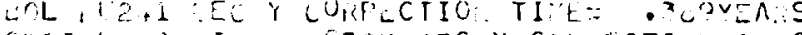

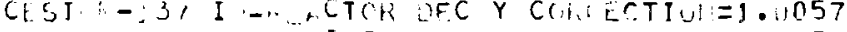

- - -
ASSIIMHTIORS

CESIUA- 37 HLF LIFE $=29.60(.1 \cup)$ YEAKS

FU24 1 H. LF LIFE $=14.5(0.5)$ Y R.R

ALPHIA241 $=.370(.030)$ 


\section{ELEMENI 137 ANALYSIS OF THE SAMPLE 36.00 INCHES FHOL THE TOP OF THE MIDDLE ROD}

I SOTOPIC INVENTOKY

\begin{tabular}{|c|c|c|c|c|}
\hline SOTOF & HOL $10 \mathrm{M}$ & EOL MASS RAYIO & $E O L \quad(A / 0)$ & $E C L(G M)$ \\
\hline-234 & $.4976-04$ & $.5169-04(.22,-05)$ & $.0051(.0002)$ & $.5013-04(.225-05)$ \\
\hline-235 & $.7076-02$ & $14-02(.333-04)$ & $.5878(.0033)$ & $-04 !$ \\
\hline$U-<3 \epsilon$ & . Un00 & $.2145-03(.487-05)$ & $.0218(.0005)$ & $.2147-03(.484-05)$ \\
\hline $\begin{array}{l}U-Z 3 E \\
T O T A L\end{array}$ & $\begin{array}{l}.9981-00 \\
.9252-00\end{array}$ & 1.0000 & 33 ) & $\begin{array}{r}141-031 \\
137-031\end{array}$ \\
\hline
\end{tabular}

\section{$150 T O F E$}

PUढ3

$\mathrm{PUCHC}^{\circ}$

PU⿻41

fiv 242

SOL $(A / 0)$ $50.1830(.0350)$
17.27301 .0430
2.31001 .0060

$.23401 .0005)$ EOL

MASS RATIO

$9972-00(.137-03)$

ISOTOFL

$\mathrm{HU}_{2} 3 \mathrm{C}$

$\mathrm{U}_{<40}$

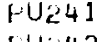

PU:42

IOTRLS

ISUiCPi

$\mathrm{u}=3 \mathrm{as}$

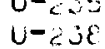

WCis

TUTAL

ATTO

EOL $(A>0)$

$.3193-00(.106-02) \quad 22.8342(.0591)$

$4.9654(.0272)$

$.9605-02(.108-03) \cdots .6869(.6077)$

BOL (GM)

$\begin{array}{rr}E L(G M) & B 0 L(G M) \\ .3758-02(.8520-04) & .3825-02(.1929-04) \\ .1205-02(.2730-04) & .8275-03(.4641-05) \\ .2631-03(.6151-05) & .1132-03(.0441-06) \\ .3655-04(.9246-06) & .1130-04(.6165-07) \\ .5263-02(.1191-02) & .4777-02(.2400-04)\end{array}$

F I 5 S O N 5

$\cdot 2290-0,100316-64$
BURNIIP (MWD/MTM)

$$
\begin{array}{r}
1054.2 \\
145.1 \\
967.8 \\
67.4
\end{array}
$$

67.4
2234.5
FISSION YIELD

$.0620(.0014)$

$.0626(.0060)$

$.0674(.0019)$

$.0660(.0033)$
DEL

$.0609(.0061)$

$.0766(.0077)$

$.0799(.0080)$
NET PRODUCTIOH: (GM) $.3670-06(.225-05)$ $-.1316-02(.329-04)$ $.2147-03(.484-05)$ $-.1676-02(.141-03)$

SPECIFIC ACTIVITY (D/M/GM) $.13616+12(.170+09)$ $.50130+12(.300+10)$ $.87000+10(.200+09)$

NET PRODUCTION (GP) $-.6689-04(.8736-04)$ 3776-03(.2769-04) $.1499-03(.6185-05)$ 2525-04(.9267-05) $4859-03(.1215-03)$

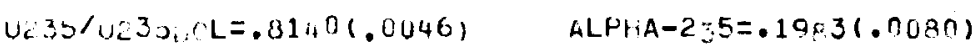

ALPHA-239= $.6323(.15 A 9)$

PU239 FORMED FROM U238 $=.1535-02(.14-03)$
INPUT DATA

CFSIL-137 CECAYS $=.1960+1[(.3920+08) D / M / M L$

PIJ DECAYS $=.13=1+U 9(.2662+7) \mathrm{D} / \mathrm{M} / \mathrm{ML}$

PERCENT DECAYC OY PIIZ38= .30

SAMPLE T=37.736GM CL D WT $=7.157 \mathrm{GM}$

SO' L! IIR,

PERCENT DECAYS OY PIIZ38= C.30

SAMPLE $T=37.736 G \mathrm{MI} \quad C L D W T=7.1576 \mathrm{M}$

SOLUTIO,: VOL $=54.9 \mathrm{ML}$

TINE OUT OF RFACTOR=.712YRS

BOL FU241 CEC:Y CORRECTIO: TINE= .389YEAPS

CESIUM-137 I IVREACTOR DECAY CORFECTION=1.0057

---DLAITITIES ENCLOSED BY PARENTHESES ARE ONE STANOARD DEVIATION ERRORS-----
ASSIJMPTIONS

CESIUM- 137 HALF LIFE $=29.68(.10)$ YEARS

PU241 HALF LIFE $=14.5(0.5)$ YEARS

ALPHA241 $=.370(.030)$

'ALPHAZLIII='.370'(.030) 
ELENEN!T $: 1.7 \%$ ALALYSIS OF THE SAMPLE 45.00 INCHES EROMLIHE TOP OF THE MIDDLE ROD

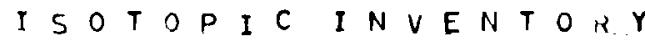

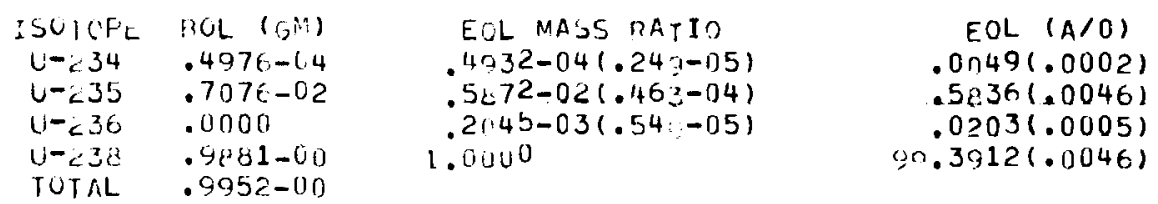

TUTAL $\quad .9952-00$

\section{FOL $\because A S S$, $\triangle A T Y O$ \\ 1.11000 \\ $.3079-00(.940-03)$ \\ $.0204-01(.344-03)$ \\ $.8540-02(.920-04)$}

E.L (GM)

.77,7-02 (.8487-04)

. 11 9-02(.2623-04)

$.245-03(.5662-05)$

$.321 .0-04(.8126-06)$
$.51,0-02(.1171-0 \times)$
EOL $(G N)$

$.5720-02(.458-04)$

$.2000-03(.545-05)$

$.9913-00(.142-03)$
$.4785-04(.248-05)$

$.9974-00(.134-03)$
NET PROUUCTIOH (GM)
-.1916-05(.248-05)
$-.1356-02(.45(-04)$
$.2000-03(.545-15)$
$-.1479-02(.142-03)$
$-.2639-02(.134-1) 3$

SPECIF IC NCTIVITY $(0 / \mathrm{M} / \mathrm{GH})$

EOL $(A / 0)$

$72.4294(.4535)$

$22.3026(.4537)$

$4.6491(.0253)$

$.6186(., 066)$

BOL ( jiM)

$.0825-02(.1,29-04)$

$.8275-03(.4041-05)$

$.1132-03(.0441-06)$

$.1130-04(.0 .05-07)$

$.4777-02(.2400-04)$

F I 5 S I O HS

isuicf

$4-25$

$1 j-<3$

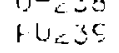

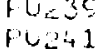

TOTILD HUTENP (.WT /MTH)

1106.2
140.1
1.70 .2
50.3

140.1
+70.2

50.3
2.172 .8

\begin{abstract}
$\therefore I S I C S$ (Gis)
- $125=02(.040-04)$

- 3ecéte-0?(-7404-64)

- $5 x 7:-0)+(0140-05)$
\end{abstract}

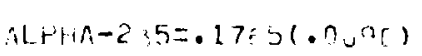

.1.PHA-239= .609, (.1754)

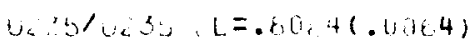

ASSIIMPTIONS

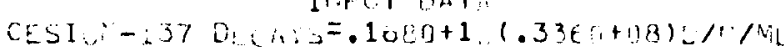

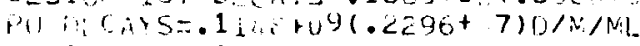

PEAC TA LACAY Y PIL33=.30

SARFL $T=32.1$ Nin CL D WT $=.352 \hat{L}^{\circ}$

CESIUH -137 HALF LIFE $=29.68(.10)$ Y! ARS

PU241, LF LIFE $=14.5(0.5)$ YEAPS

SILLIIIO VCL $=4 ;, 1: 4$

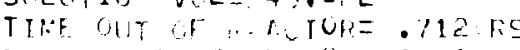

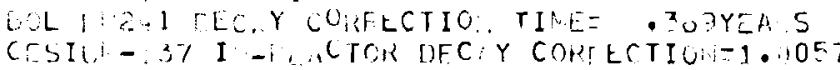

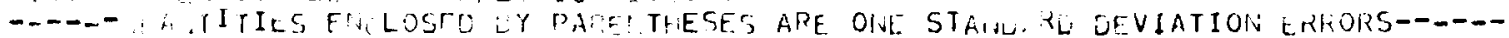


ELEMEIT 2137 ANALYSIS OF IHF SAMPLE 55.00 INCHES FRON THE TOP OF THE MIDDLE ROD

I SO TOPIC INVENTOBY

$\begin{array}{ll}\text { ISUIOPC } & \text { BOL }(G M) \\ U-\angle 34 & .4976-04 \\ L-\angle 35 & .7076-02 \\ U-\angle 36 & .0000 \\ U-\angle 38 & .9381-00 \\ \text { TUTAL } & .9952-00\end{array}$

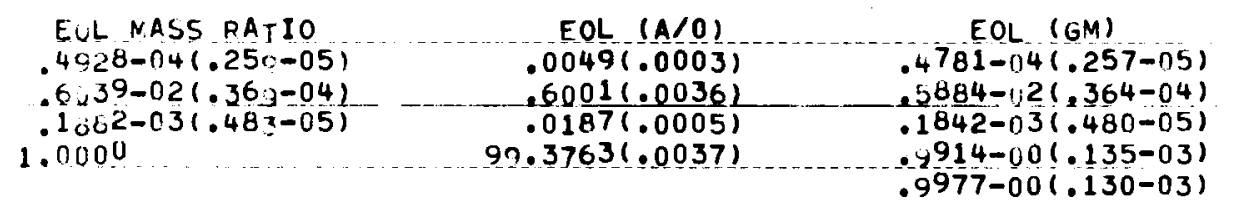

$$
\begin{aligned}
& \text { NET PRODUCTION (GN) } \\
& -.1953-05(.257-05) \\
& -.1192-02(.364-04) \\
& .1842-03(.460-05) \\
& -.1328-02(.135-03) \\
& -.2339-02(.130-03)
\end{aligned}
$$

\begin{tabular}{|c|c|c|c|c|}
\hline $\begin{array}{l}\text { ISO1 UPE } \\
\text { PUट39 } \\
\text { PUC40 } \\
P U C 41 \\
P U 242\end{array}$ & $\begin{array}{r}101(4 / 0) \\
0.1830(.1350) \\
17.2730(.0430) \\
2.0100(.060) \\
.2340(.0005)\end{array}$ & $\begin{array}{l}\text { EOL PIASS RATIO } \\
1.0000 \\
.3051-00(.833-03) \\
.6277-01(.0363-03) \\
.8496-02(.017-04)\end{array}$ & $\begin{array}{l}72.5411(.0485) \\
22.1316(.0475) \\
4.7110(.0266) \\
.6163(.0066)\end{array}$ & $\begin{array}{c}\text { SPECIFIC ACTIVITY }(0 / N / G) \\
.13616+12(.170+09) \\
.50130+12(.300+10) \\
.57600+10(.100+09) \\
.87000+10(.200+00)\end{array}$ \\
\hline $\begin{array}{l}\text { ISUTCFE } \\
\text { PUE SS } \\
\text { PUE } \\
\text { PUE4 } \\
\text { PUE42 } \\
\text { TCTFLS }\end{array}$ & & $\begin{aligned} & E C L(G N) \\
. & 37,8-02(.8393-04) \\
. & 1136-02(.2570-04) \\
. & 24,8-03(.5679-05) \\
. & 31-0-04(.7991-06) \\
. & 5118-02(.1157-02)\end{aligned}$ & $\begin{array}{c}\text { BOL }(G M) \\
.3825-02(.1929-04) \\
.8275-03(.4041-05) \\
.1132-03(.6441-06) \\
.1130-04(.6165-07) \\
.4777-02(.2400-04)\end{array}$ & $\begin{array}{l}\text { NET PFOUUCTION (GN) } \\
-.1174-03(.8612-04) \\
.3385-03(.2612-64) \\
.1296-03(.5716-05) \\
.2059-04(.8014-06) \\
.3412-03(.11142-03)\end{array}$ \\
\hline
\end{tabular}

F I 55 I 0 N 5

ISCIOF $U-c 35$

FISIO S (GN) - $1106-0<(-3072-(44)$ $0.1201-0 \geqslant(101288-64)$ - $1<01-0 z(1208-64)$ $.0691-03(.0416-04)$ $.5543-04(.+985-05)$
$.1 .9(5-02(.5472-04)$
PURNI:P (NWR /MTM)

05.3
125.6
707.7
55.0

FISSION YIELD $.0626(.0014)$ .062 u $(.0060)$ $.0674(.0019)$ 1043.6

UK35/153 $20.1=.315(.0051) \quad$ ALPHA-2 $55=.1840(.0050)$ INPUT DATA

CESIUN-137 CECAYS $=.1710+1 \quad(.3420+08) 0 / N / M L$

PU CECAYS $=.1279+U^{9}(.<558+i 7) 0 / \mathrm{M} / \mathrm{ML}$

PERCENT UECAYS OI PUL3B=

PERCELT UECAYS ON PU23B
SAMPLE $: T=36.915 G^{M} \quad C L, P W T=6.18 B G M$

CU DECAYS $=.1279+U 9(.<558+7) 0 / M / M L$

PERCEINT UECAYS II PU238= 080

SAMPLE :T T $=36.315 G^{M} \quad C L A W T=6.18 B G M$

SOLUTIOI: VCL $=55.2 \mathrm{ML}$

TIME OUT OF REACTOR= .712YRS

EOL HU241 LEC Y CORRECTIOJI TIMEE -369YEABS

CESILM-137 I -REACTOR DEC Y CORPECTION=1.0057

-

$\begin{array}{cc}\text { OEL } & \text { MEV/FISSIOI } \\ .0609(.0061) & 202 \\ .0766(.0077) & 209 \\ .0799(.0080) & 211 \\ \end{array}$

ALPHA-239= $.6354(.1877)$

P.J239 FORIAED FROM U238 $=.1205-02(.13-0.3)$
CESIUM- 37 HALF LIFE $=29.681 .10)$ YEARS

PU241 HIF LIFE $=14.5(0.5)$ YEARS

ALPHA241 $=.370(.030)$

PUT241

$(0.5)$ YEARS

ALPHA241 $=.370(.030)$ 
ELEMEITT :.2.7 ANAL ;SIS OF THE SAMPLE 65.00 INCHES ERU.. THE TOP OF THE MIDLLE ROD I $S O T O P I C$ I N V E N TOMY

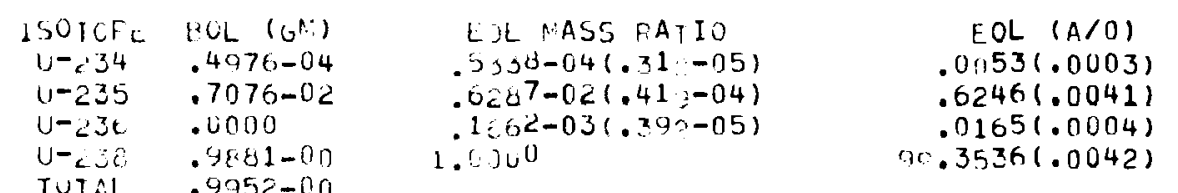

TUTी

$.9952-00$
EOL $(G M)$ $. \sqcup 179-04(.316-05)$ $.0126-02(.413-04)$ $.1626-03(.390-05)$ $.4916-00(.133-03)$ $.4916-00(.133-03)$
$.4980-00(.126-03)$
INET PROOUCTIOH, $\left(G, v_{i}\right)$ $.2030-05(.316-05)$ $-.0490-03(.413-04)$ $.1626-03(.390-05)$ $-.1173-02(.133-03)$ $-.1968-02(.120-0) 3)$
SUICPL

$r U^{2}+i r$

मu: 1

IUT, LS

$$
\begin{aligned}
& \text { lsulce. } \\
& \text { (1) }-4 \\
& \text { 11- } 11-2 \\
& \text { rusis } \\
& 1 \text { U⿺⿻一𠃋十 } 1 \\
& \text { TCIF.L, }
\end{aligned}
$$

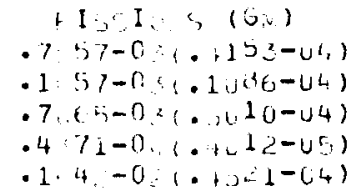

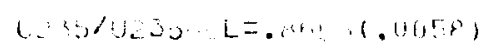

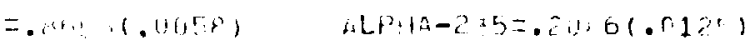

$$
\begin{aligned}
& \text { EOL ASS AATIO } \\
& 1.0000 \\
& .2866-00(.832-0=) \\
& .5691-01(.335-03) \\
& .7217-02(.017-04)
\end{aligned}
$$

$.37 \cdot 9-02(.6528-04)$

. $10.5-02(.2453-04)$ $.27-4-04(.6062-0 \%)$
$.51 .5-02(.1154-02)$

F I S S I O H S

$$
E L(\mathrm{~cm})
$$
$.2238-03(.5238-05)$

EOL $(A, 0)$ $73.9291\left(.4^{498}\right)$

$21.1844(.490)$

$4.3530(. .251)$

$.5335(., 060)$

E.OL (GM)

$.3225-02(.1 .29-04)$

$.0275-03(.4,41-05)$

$.1132-03(.62441-06)$

$.1130-04(.5205-07)$

$.4777-02(.2400-04)$

$$
\text { ivput dar: }
$$

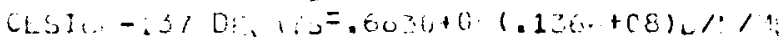

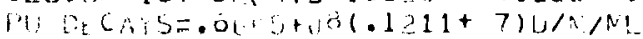

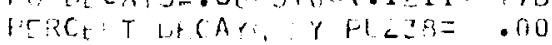

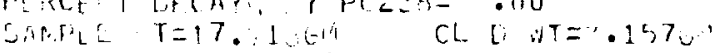

SHLULO $\because C L=1,1 \mathrm{ML}$

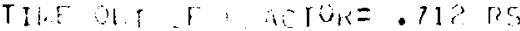

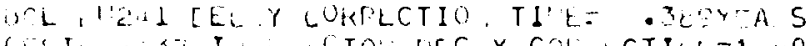

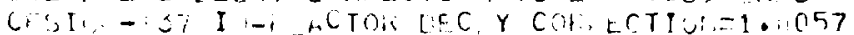

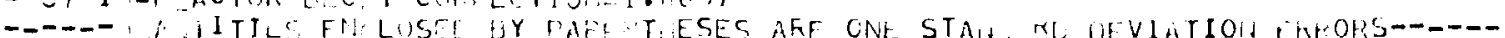

FISO OH YIHL

.0j2:(.01) 14)

$.0620(.0060)$

$.067 .1 .0019)$

$.0004(.0033)$
$\therefore\left(L_{14}-23 y=.605 .(.2140)\right.$
LEL.

$0700(-1077)$

$.0700(.1077)$
$.0799(.0080)$
SPECIFIC ACTIVITY $(O / M / G(4)$ $.1301 t+12(.170+(j))$ . $5.1130+12(.3,0+10)$ $.57000+10(.110+00)$ . $67000+10(.200+(4.1)$

NET PAOLLCTLOII (W) $-.5013-04(.8744-014)$ $.2571-03\left(.2447-\left(1 t_{4}\right)\right.$ $.1106-03(.52 .77-(19)$ $.1+24+04(0,14,1)-0 t)$ $.3277-03(.117 n-0 ;)$
SAE"/FISSIO!

2,6

211 
FLEMEIT 5127 ANALYSIS OF THF SAMPLE 75.00 INCHES FROM THE TOP OF THE MIDDLE ROD

I SOTOPIC INVENTOKY

$\begin{array}{ll}\text { ISUIOFE } & 1 \mathrm{OL}(\mathrm{GM}) \\ U-634 & .4976-04 \\ U-\angle 3 S & .7076-02 \\ U-236 & .0000 \\ U-23 F & .9881-00 \\ \text { TOTAL } & .9952-00\end{array}$

$\begin{array}{ccc}\text { EQL NASS RATIO } & \text { EOL }(A / 0) & \text { EOL }(G M) \\ .5407-04(.294-05) & .0054(.0003) & .5305-04(.292-05) \\ .6539-02(.42 .04) & .6495(.0042) & .6373-02(.417-04) \\ .1202-03(.355-05) & .0127(.0004) & .1255-03(.353-05) \\ 1.0000 & 9.3323(.0042) & .9918-00(.129-03)\end{array}$

NET PRODUCTION (GM)

$.3292-05(.292-05)$

$-.7028-03(.417-04)$

$.1255-03(.353-05)$

$-.9867-03(.129-03)$

$-.1565-02(.122-03)$

ISOTOFL

PUCZO

PU: 40

PU⿻ 4

PU: $4:$

$150 \mathrm{iCN}$

PUदz?

PU: 40

PU:4 1

ru< 4 ?

TUTALS

\begin{tabular}{|c|c|c|}
\hline $\begin{array}{r}.01(A / 0) \\
0.1830(.0350) \\
17.2730(.0430) \\
2.2100(.1 .060) \\
.2340(.0005)\end{array}$ & $\begin{array}{l}\text { EOL MASS RATIO } \\
1.0000 \\
.2728-00(.857-03) \\
.5085-01(.280=03) \\
.6045-02(.656-04)\end{array}$ & $\begin{array}{r}75.1069(.0514) \\
20.4877(.0514) \\
3.9514(.0222) \\
.4540(.0049)\end{array}$ \\
\hline & $\begin{array}{l}E L(G M) \\
.3 B_{1} B-02(.8616-04) \\
.1043-02(.2360-04) \\
2020-03(.4718-05) \\
.2331-04(.5848-06) \\
.5077-02(.1147-03)\end{array}$ & $\begin{array}{r}\text { BOL (GM) } \\
.3825-02(.1929-04) \\
.8275-03(.4641-05) \\
.1132-03(.0441-06) \\
.1130-04(.6165-07) \\
.4777-02(.2400-04)\end{array}$ \\
\hline
\end{tabular}

F I S I I N $S$

SPECIFIC ACTIVITY $(\mathrm{O} / \mathrm{N} / \mathrm{G}(\mathrm{P})$ $.13616+12(.170+0(3)$ $.50130+12(.300+10)$ $.57600+10(.1100+09)$ $.87000+10(.200+09)$

NET PRODUCTION (GM)

-.1675-04(.8829-04)

$.2157-03(.2405-04)$ $.8483-04(.4762-05)$ $.1201-04(.5841-06)$ $.2998-03(.1172-03)$

I (O) $S$ (G/V)

IsOTOF

$v-235$

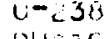

PU:

गि1

TOTALS

(0)

- 3232-04(.3061-05)

$.3236-04(.3061-65)$
$.126 n-02(.3513-04)$
EUPNIP (. WO/MTM)

553.7

90.5

568.4

1234.7

U235/UC3DI:L $L=.0007(.0050)$

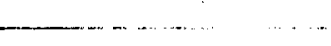

LEL
$.0609(.0061)$

$.0628(.0014)$

$.062(.0060$

$.0004(.0033)$

$.0766(.0077)$

$.0799(.0090)$
MEV/FISSIO:
202
209
211

PU239 FORINED FROM U238 $=.9084-03(.13-03)$

DU CLCAYS OLCAYS $=.8020+00(.1724+08) D / M / M L$

(1) $1929+7) D / M / M L$

PERCEITT UECAYS LY PUC3B $=7.10$

SANPLE T $T=30.5,05 \mathrm{GM} \quad \mathrm{ML}, \mathrm{D} W \mathrm{WT}=6.204 \mathrm{GM}$

ALPHA-239 $=.605 C^{\prime}(.2607)$

NS

CESIUM-I 37 HALF LIFE $=29.68(.10)$ Y TARS

PU241 HILF LIFE $=14.5(0.5)$ YEARS

ALPHA241 $=.370(.030)$

SOLUTIO: VOL $=54.7 \mathrm{ML}$

TIME OUT OF RPACTOR=.712YRS

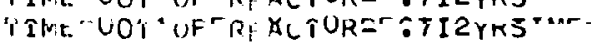

BOL Pli241 CECIY CURRECTIO : TIME = 389YEARS

CFSIUN:-137 IIJ-REACTOR DEC/Y CORFECTIOHI $=1.0057$

----OI, AiNTITIES ENCLOSFD BY PAREIITHESES ARE ONE STANGARD DEVIATION ERRORS----- 
ELLME:T .1.7 AIAL, SIS OF THE SAIPLE 85.00 INCHES F.RUII THE TUP GF THE MIDULE ROD

I : O T O P I C I N VENTO

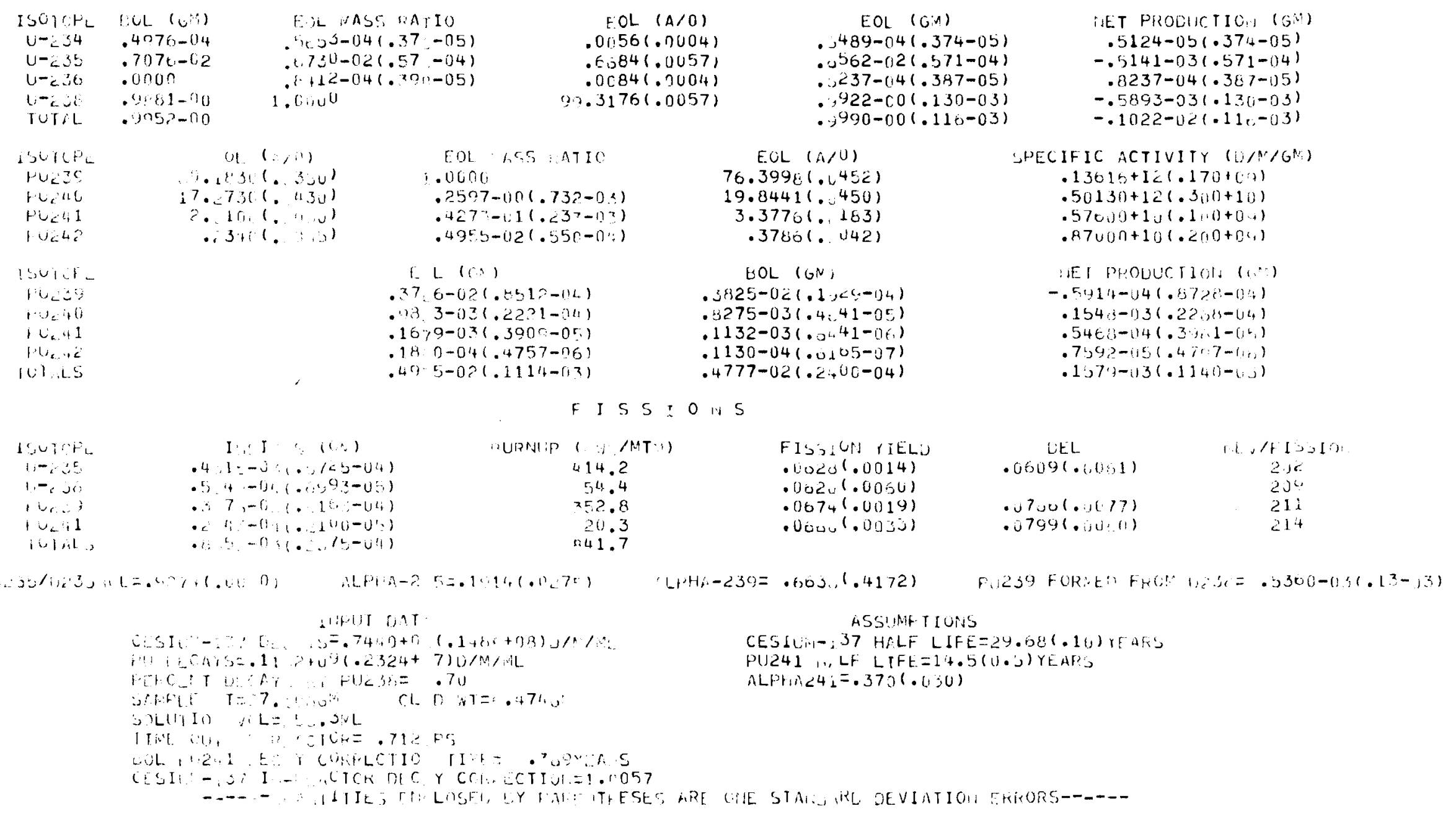




\section{ELENERT ED D P ANALYSIS OF THE SAMPLE 3.00 INCHES FRON THE TOP OF THE OUTSINE ROC}

\section{ISOTOP I C I N VENTOKY}

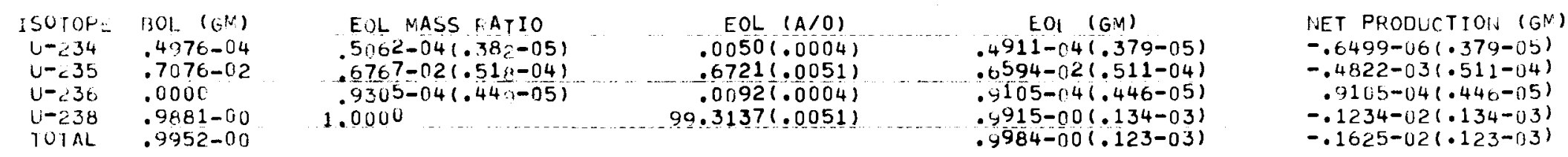

\begin{tabular}{|c|c|c|}
\hline $\begin{aligned} & E O L \\
& .18301 \\
& .27301 .0 \\
& .31006 \\
& .23406\end{aligned}$ & $\begin{array}{l}\text { EOL MASS RATIO } \\
1.0000 \\
.2611-00(.703-03) \\
.4143-01(.230-03) \\
.4988-02(.535-04)\end{array}$ & $\begin{array}{r}\frac{76 \cdot 3}{19 \cdot 9} \\
3 \cdot 3 \\
.3\end{array}$ \\
\hline
\end{tabular}

SPECIFIC ACTIVITY $(\mathrm{C} / M / G \mathrm{~V})$

$.13616+12(.170+09)$

$.50130+12(.300+10)$

$.57000+10(.130+09)$

$.87000+10(.200+04)$

EOL (GM)

$.3864-02(.8733-04)$

$.1013-02(.2290-04)$

$.1698-03(.3962-05)$

$.1952-04(.4883-06)$

$B O L^{-}(G M)$

PU:39

PU⿺4⿻一𠃋十

Fu⒋ 42

TUTALS

ISC1OF"

$U-c .45$

$0-238$

Plic 39

Plis

TUTALS

U235/U23 3

F I S S I O NS $\ldots \ldots$
FISSICS $\left(G_{i}\right)$ .3911-0?(.5131-04) $.6993-04(.7456-05)$ $.8583-02(.0399-04)$ $.2211-0.4(0.2(29-05)$ $.1340-0 \cdot(.3974-04)$

$.3825-02(.1929-04)$

$.8275-03(.46,41-05)$

$.1132-03(.6441-06)$

$.1130-04(.0+65-07)$

$.4777-02(.2400-04)$

NET PRODUCTION (GM) .3906-04(.8943-04) $.1057-03(.2337-04)$ $.5658-04(.4014-05)$ $.8214-05(.4921-0 n)$ $.2896-03(.1169-03)$
FISSION YIELU $.0626(.0014)$ $.0626(.0060)$ $.0674(.0019)$

$.060 \mathrm{v}(.0033)$

$\begin{array}{cl}(\text { BWD/MTH) } & \text { FISSION YIELU } \\ \times 75.4 & .0620(.0014) \\ 67.6 & .0620(.0060) \\ 846.2 & .0674(.0019) \\ 21.9 & .0604(.0033) \\ 1311.1 & \end{array}$

ALPHA-239 $=.3177(.1731)$

$\begin{array}{cc}\text { DEL } & \text { MEV/FISSI(ii) } \\ .0491(.0049) & 202 \\ .0563(.0056) & 209 \\ .0604(.0060) & 211 \\ & 214\end{array}$

P1239 FORMED FROM UZ3E= .1170-02(.13-03)

INPUT DAT:

CESIUM-137 DECAYS $=.8070+09(.1614+08) \cup / N / M L$ PU [LLCAYS $=.841 .1+68(.1688+1.7) \mathrm{D} / \mathrm{M} / \mathrm{ML}$

PFRCENT OECAYS SY PUZ38= 90

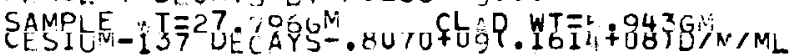

PU RILCAYS $=.84 i .1+68(.1688+.7) \mathrm{D} / \mathrm{M} / \mathrm{ML}$

PFRCENT OECAYS IY PUC38 $=, 90$

SAMPLE WT=27.7066M $\quad C L A D W T=\$ .94 \times 6 \mathrm{~F}$

ASSUMPTIONS

CESIUM- 37 HALF LIFE $=29.68(.10)$ YEARS

PU2 41 HALF LIFE $=14.5(0.5)$ YEARS

ALPHA241 $=.370(.030)$

LE $\ 1 U M-I O I$ HALF L1FE $=29.081 .1 U$ ITEAKS

PU2 41 HALF LIFE $=14.5(0.5)$ YEARS

ALPHA241 $=.370(.030)$

SOLUTIOH VOL $=559.2 \mathrm{ML}$

TIME OUT OF $A$ ACTOR $=1.055 Y$ YR

BOL PU241 OECIY CORRECTIO: TIME = .389YEARS

CESIUM-137 II.-REACTOK DECAY CORPECTION $=1.0057$

----GLATITIIES ENCLOSED BY PARENTHESES ARE ONE STANDARD DEVIATION ERRORS----- 
ELEMENT 5.197 ... ANAL ISIS OF IHE SAMPLE 13.00 INCHES FRQV _.THE TOP OF THE OUTSIDE ROD

I SOOTOP I C I N V.ENTOKY

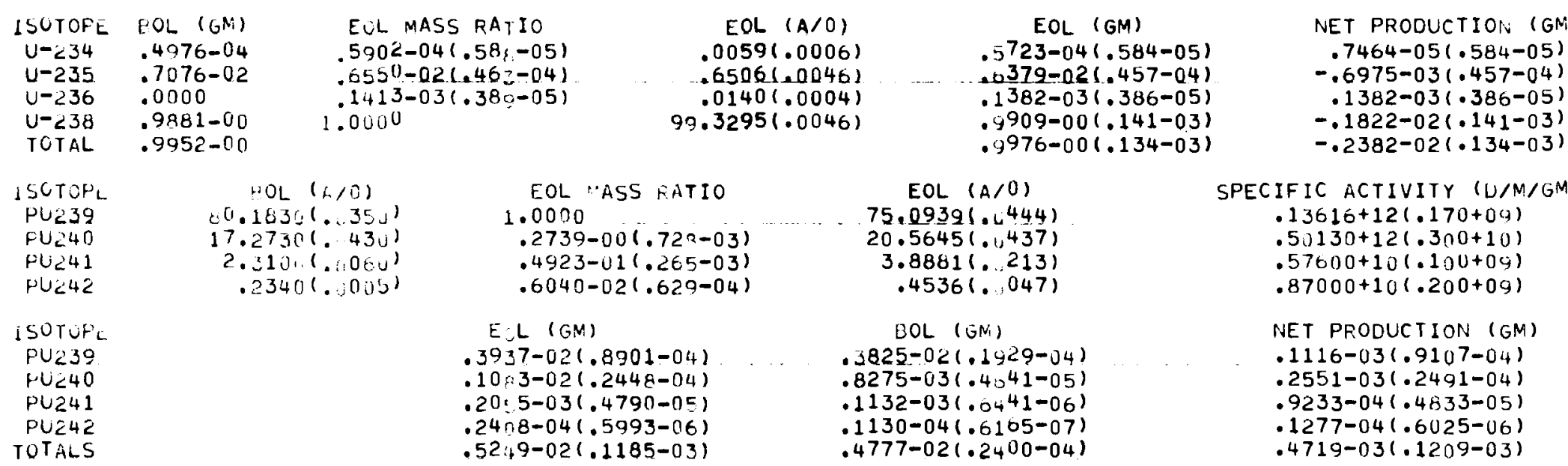

F. I $\$$ SION $\mathbf{S}$

\begin{tabular}{|c|c|c|c|}
\hline $\begin{array}{l}\text { ISOTOPE } \\
U-23 E \\
L-236 \\
H U \angle Z \\
\text { PUC41 } \\
\text { TOTALS }\end{array}$ & $\begin{array}{c}\text { FISSI }\left(S\left(G_{N}\right)\right. \\
.5583-0 \times(.4587-04) \\
.9854-04(.1016-04) \\
.1224-02(.7133-04) \\
.3438-01.025-05) \\
.1316-0 ?(.5076-04)\end{array}$ & $\begin{array}{c}\text { FURNIF }(1 \% \text { WDTMA) } \\
536.4 \\
96.6 \\
1207.0 \\
34.1 \ldots \\
1974.2\end{array}$ & $\begin{array}{l}\text { FISS:ON YIELD } \\
.0620(.0014) \\
.0624(.0060) \\
.067+(.0019) \\
.066(.0033)\end{array}$ \\
\hline
\end{tabular}

UC35/U23JiOL $=.9014(.0064) \quad$ ALPHA-235=.2482(.0221)

ALPHA-23G $=.3223(.1251)$

$\begin{array}{cc}\text { DEL } & \text { MEV/FISSION } \\ .0491(.0049) & 202 \\ & 209 \\ .0563(.0056) & 211 \\ .0604(.0060) & 214\end{array}$

INPUT DAT:

CESI IV-137 DECAYS $=.1220+1,(.2440+08) 0 / \mathrm{M} / \mathrm{ML}$

PU O.LCAYS $=.94 .7+08(.1891+7) \mathrm{D} / \mathrm{M} / \mathrm{ML}$

PERCENT DECAYS E.T PU238 $=.30$

SAIVPLE: $T=27.300 \mathrm{GM}$

$C L: D W T=4.930 \mathrm{OL}$

PI/239 FORMED FROM U238 $=.1730-02(.14-03)$

TINE OUT OF

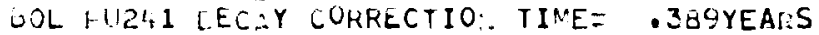

CTCIV -137 I

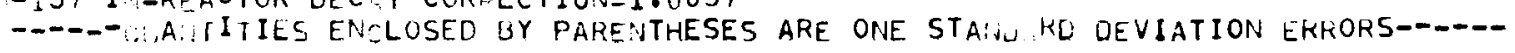


..... ELEME T 5187 ANALYSIS OF THE SAMPLE 24,00 INCHES FRO THE TOP OF THE OUTSIDE POD

I SOTOPIC INVENIOKY

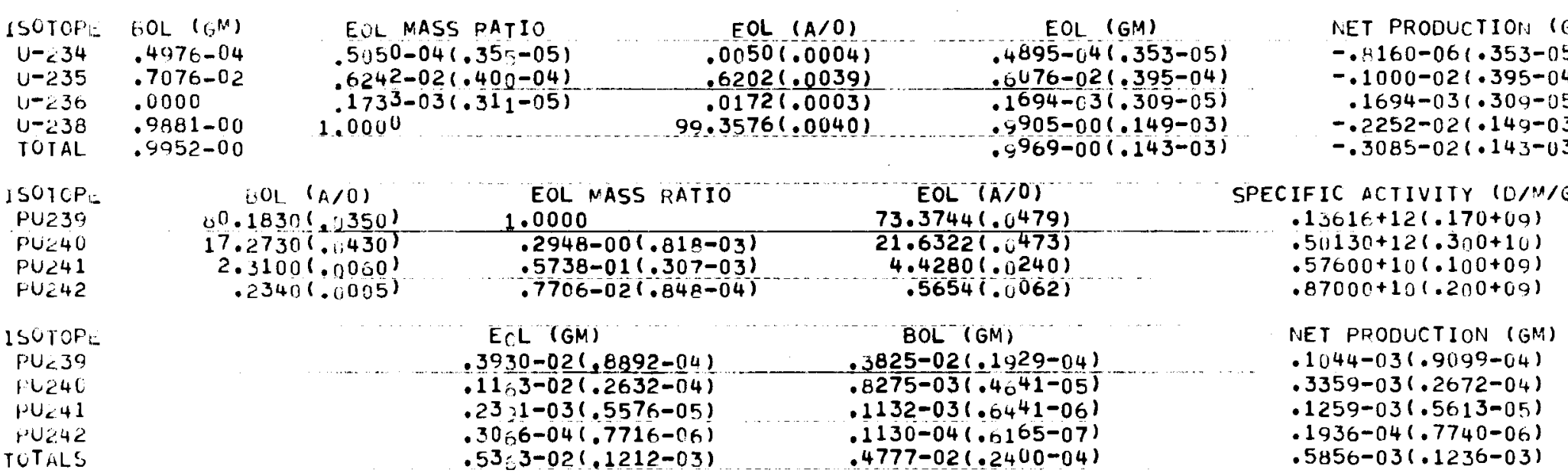

$F I S S I O N S$

\begin{tabular}{|c|c|c|c|c|c|}
\hline $\begin{array}{l}\text { ISOTCP } \\
\text { U-C35 } \\
U-238 \\
P U C 35 \\
\text { PU241 } \\
\text { TUTAL }\end{array}$ & $\begin{array}{c}\text { FIS-IOIS (GN) } \\
.8-91-03(.3959-04) \\
.1283-03(.1296-04) \\
.149(1-02(.8053-04) \\
.5210-04(.4710-05) \\
.2505-02(.7280-04)\end{array}$ & $\begin{array}{c}\text { FURNIP }(\because W 1 / M T M) \\
795.9 \\
125.8 \\
1474.5 \\
51.7 \\
2447.9\end{array}$ & $\begin{array}{l}\text { FISSION YIEL }) \\
.0620(.0014) \\
.0620(.0060) \\
.0674(.0019) \\
.0664(.0033)\end{array}$ & $\begin{array}{c}\text { DEL } \\
.0491(.0449) \\
.0563(.0056) \\
.0604(.0060)\end{array}$ & $\begin{array}{c}\text { MEV/FISSIO } \\
202 \\
209 \\
211 \\
214\end{array}$ \\
\hline
\end{tabular}

UL3)/U23,ILL $=.85,7(.0056) \quad$ ALPHA-2:5 $=.2062(.010 \mathrm{~F})$

ALPHA-239= $.35 c \mathrm{co}(.1044)$

P.:239 FORIED FROM U238 $=.2133-02(.15-0.3)$

\section{INPUT OAT:}

CESIUN-137 DECAYS $=1680+13(.3360+08) \mathrm{D} / \mathrm{M} / \mathrm{ML}$

PU DECAYS $=.1$ ก $1+1+09(.2102+07) 0 / \mathrm{M} / \mathrm{ML}$

PERCEIT LECAYS GY PU238= 9.70

SAMPLE $T=29.239 \mathrm{GM} \quad \mathrm{CL} D W T=5.361 \mathrm{GM}$

PU DECAYS $=1$ 1OC.1+09(.2102+07)D/M/ML

PERCET LECAYS GY PU238 $=9.70$

SAMPLE $\because T=29.239 \mathrm{GM} \quad C L A D W T=5.361 \mathrm{GM}$

SOLUTIOI: VOL $=253.7 \mathrm{ML}$

TIME CUT OF REACTOR=1.055YRS

BOL PU241 OEC, Y CORRECTIOI: TIME = 389YEAFS

CESIUM-137 II,-REACTOR DECAY CORFECTIONI $=1.0057$

\section{ASSUMPTIONS}

CESIUM- 37 HALF LIFE $=29.68(.10)$ YEARS

PU241 HALF LIFE $=14.5(0.5)$ YEARS

ALPHA2 $41=.370(.030)$

PÜ241 HALLF LIFE $=14.5(0.5)$ YEARS

ALPHA241 $=.370(.030)$ 


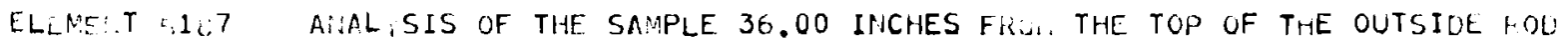

I $S O T \cap P$ I C I NVENTOKY

\begin{tabular}{|c|c|c|}
\hline & & \\
\hline ISUTOPE & $\mathrm{BOL} \quad(6 *)$ & ELL NASS RAIIO \\
\hline$L-234$ & $.4076-04$ & $.5114-04(.33-05)$ \\
\hline $1-235$ & $.71176-02$ & $.61 c^{b}-0<(.527-04)$ \\
\hline$U-636$ & .0000 & $.1735-03(.79,-05)$ \\
\hline 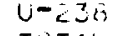 & $\cdot 9[3]-00$ & 1.0300 \\
\hline TUTAL & $.495 z-010$ & \\
\hline
\end{tabular}

isurat:

PUe3?

$\mathrm{Pu}_{\mathrm{C}} 40$

$P L^{2} 41$

Pu. 42

SOTCHL

Pucse

No: 40

Hots

Heita

TOTALS

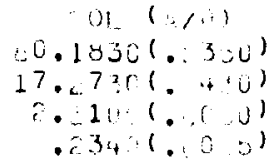

$$
\begin{aligned}
& \text { EOL A3S . .TIO } \\
& .0000 \\
& .3021-00(.30 \pi-02) \\
& .0102-01(.312-02) \\
& .3300-02(.001-02)
\end{aligned}
$$

E. L. (ON)

.3? $0-02(.3577-04)$

. 11 a-0?(.c50t-j!l)

- $643-0.3(.5707-105)$

-32.2-04(.306?-Df)

$.227-02\left(.117^{0}-02\right)$

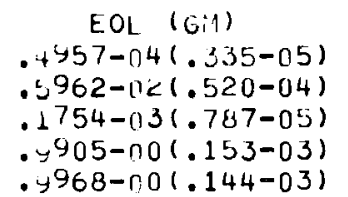

$E(L(n) 0)$

$72.7431(.0465)$

$21.9779(.459)$

$4.6064(., 242$

$.6107(.065)$

BOL (GN)

$. \$ 825-0<(.1 y<9-04)$

.0275-03(. . 4 41-0s)

$.1132-03(.+41-06)$

$.1130-04(.0105-07)$

$.4777-02(.2400-04)$
RET PRODLETIOH (GN) $-.1984-08(.335-05)$ $-.1114-02(.520-04)$ $.1754-03(.707-05)$

$-.2221-02(.153-03)$

\section{F I S S I O NS}

\section{SUIOFL}

$v-c 35$

$4-236$

PU. 35

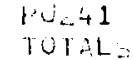

U.t. sis

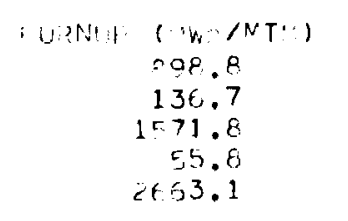

FIS. ON YIEL:

.06ż $(.0014)$

. 0uisir. 1.00

- 0uev.0060

$.067 .4(.01119)$

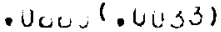

SPECIFIC ICTIVITY (LU/")

$.13616+12(.170+6+1)$

$.50130+12(.5100+11)$

$.5700(1+10(.100+0)$

$.87000+10(.2) 0+0 r)$

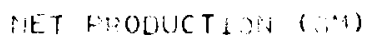

$-.3520-04(.8701-6)$

$.3<24-03\left(.264 c^{2}-(44)\right.$

. $1321-03(.57+3-i s)$

$.2491-04(.35+3-6)$

$.44(1-1) 3(.1 \%, 4-0.5)$

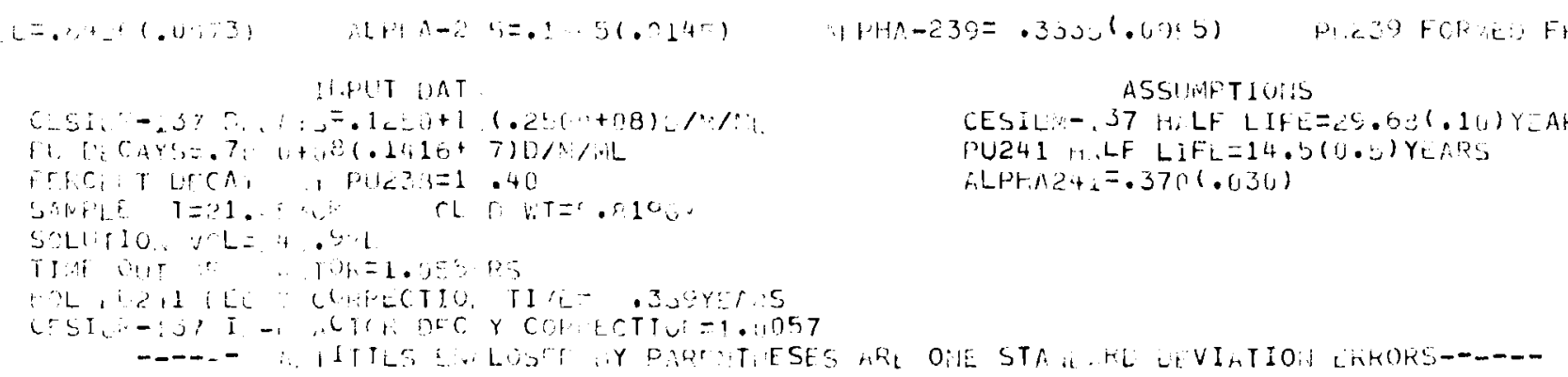

$14 \div)$

$41+1-239=.3000(.0985)$ 
ELEMENT 5137 ANALYSIS OF THE SAMPLE 45.00 INCHES FROM THE TOP OF THE OUTSIDE FOD

\section{ISOTOPIC I NVENTOKY}

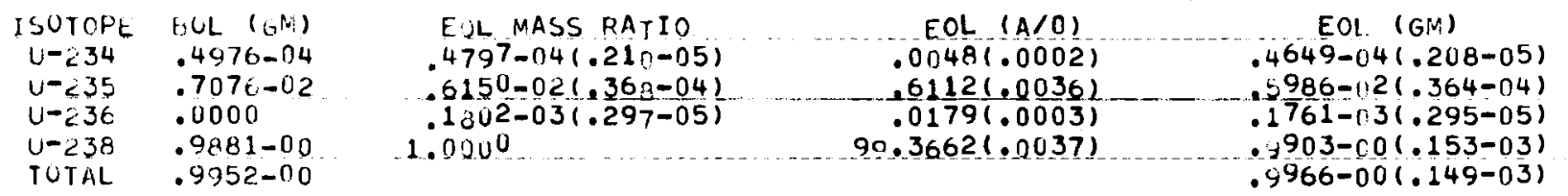

NET PIODUCTION (GM) $-.3273-05(.204-05)$ $-.1091-02(\cdot 364-04)$ $.1761-03(.295-05)$ $-.2480-02(.153-03)$
$-.3405-02(.149-03)$ $.9952-00$

\begin{tabular}{|c|c|c|}
\hline $\begin{array}{r}401(m / 0) \\
0.1830(.0350) \\
17.2730(.0430) \\
2.3100(.0000) \\
.2340(.0005)\end{array}$ & $\begin{array}{l}\text { EOL MASS RATIO } \\
1.0000 \\
.3022-00(.784-03) \\
.6147=01(.323-03) \\
.8400-02(.890-04)\end{array}$ & $\begin{array}{r}\text { EOL }(A / 0) \\
72.7167(.0458) \\
21.9714(.0449) \\
4.7011(.0249) \\
.6108(.0064)\end{array}$ \\
\hline & $\begin{array}{l}\text { EOL (GM) } \\
.3921-02(.8873-04) \\
.1100-02(.2691-04) \\
.2556-03(.5955-05) \\
.3335-04(.8333-06) \\
.5400-02(.1221-03)\end{array}$ & $\begin{array}{r}\text { BOL (GM) } \\
.3825-02(.1929-04) \\
.8275-03(.4641-05) \\
.1132-03(.6441-06) \\
.1130-04(.6165-07) \\
.4777-02(.2400-04)\end{array}$ \\
\hline
\end{tabular}

SPECIFIC ACTIVITY (L/M/GMi)

$1 S 010 \mathrm{CL}$

HU 39

$P \cup 440$

$P \cup=41$
$P \cup=42$

ISUIOFL

Pण 39

N 1440

$+4241$

Pli 42

$.5400-02(.1221-03)$

FIS SIONS

. $13616+12(.170+09)$

$.50130+12(.300+10)$

$.57600+10(.100+00)$
$.87000+10(.200+09)$

NET PFODUCTION (GM)

.9599-04(.90:1-(44)

$.36<3-03(.2731-04)$

$.1424-03(.59$ त9-05)

$.2205-04(.8356-06)$

$.6227-03(.1244-0.3)$

ISUTCFL.

$u=35$

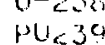

purat

TOTAL;

$$
\begin{aligned}
& \text { (IS5IOS (Gm) } \\
& .9125-03(.3051-04) \\
& \text { - } 1428-0 \times(.1437-04) \\
& \text { - } 1,74-0=(.0627-04) \\
& \text {. } 5.34+04(05311-05) \\
& \begin{array}{l}
.5 y 34-04+1.5311-05 \\
.278 \pi-0 ?(.3114-04)
\end{array}
\end{aligned}
$$

FISS ON YIELC

BUPNUP (IAWR/MTM)

$$
\begin{array}{r}
876.0 \\
140.1 \\
1650.0 \\
58.8 \\
2724.9
\end{array}
$$

2724.9

$.0620(.0014)$

$.0621(.0060)$

$.0674(.0019)$

$.060 u(.0033)$

UC.35/C30UL $=.(41.9(.0051) \quad$ ALPHA $-2 \times 5=.1951(.0037)$

ITPUT DAT:

CESILPI-137 DELAYS $=.1700+1(.340(1+08) \mathrm{D} / \mathrm{M} / \mathrm{ML}$

PUI DECAYS $=.9711+08(.1942+117) \mathrm{D} / \mathrm{M} / \mathrm{ML}$

PERCEITT DECAYS ar PUZ38 $=1 \mathrm{C} \cdot 20$

SAMPLE, $T=26.90 G^{N} \quad C L D W T=5.767 G N$

SAMPLE $T=\angle 6.90 G M$

CEL
$.0491(.0049)$
$.0563(.0056)$
$.0604(.0060)$

MEV F ISSIOI!

ALPHA-239= .350L $(.0936)$

PU239 FORMED FROM Uว38 $=.2355-02(.15-03)$

ASSUMPTIONS

CESIUM- 37 HALF LIFE $=29.68(.10)$ YEARS

PU241 HALF LIFE $=14.5(0.5)$ YEARS

ALPHA24 $1=.370(.030)$

SOLIITIO: VOL

TIME OUT UF R:ACTOR=1.055YRS

BOL FU211 LEC.1Y CORPECTIO:! TIME= -389YEARS

CESIUM-137 I -REACTOR DEC Y CORFECTION $=1.0057$

- A I I IES ENCLOSED EY FARENTHESES ARE ONE STANUMRD DFVIATION ERFORS-...- 
ELEME:IT $1: 7$ ANAL:SIS OF THE SAMPLE 55.00 INCHES FROS THE TOP OF THE OUTSIOE ROD

I $S$ O TOPIC I N V E N TOKY

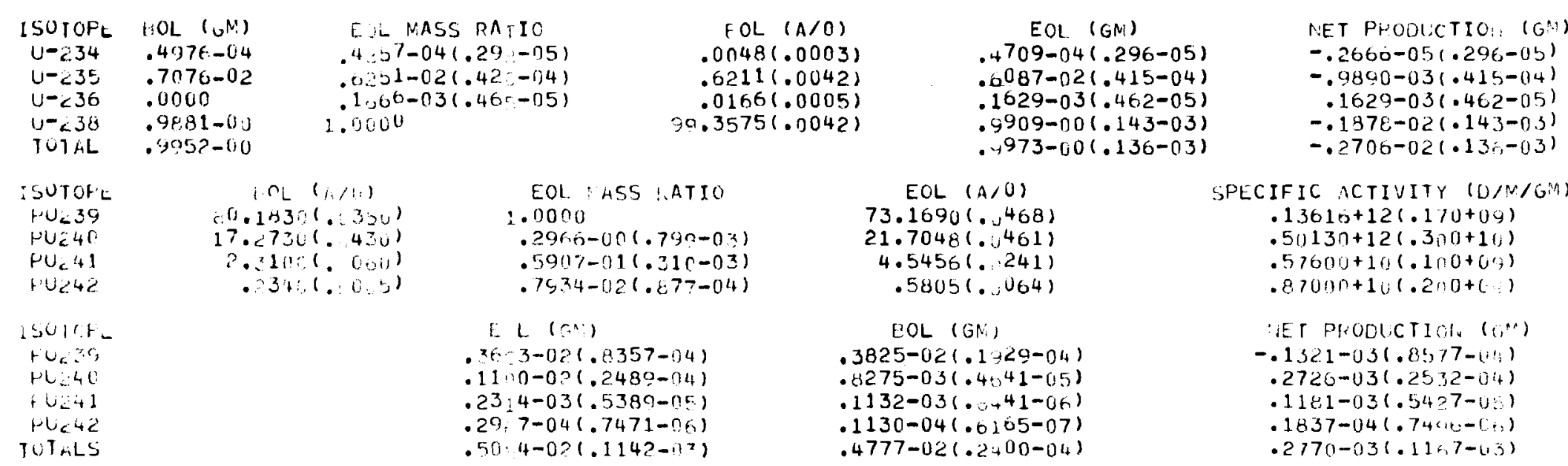

r I S S I 0 : 5

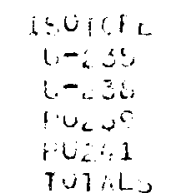

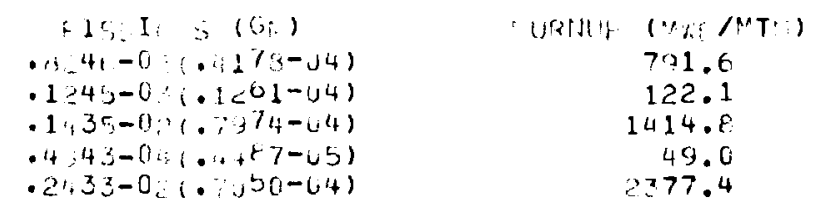

\begin{tabular}{|c|c|c|}
\hline FIsS! ON YIELij & LEL & Af ISSI \\
\hline $.002,(.0014)$ & $.6491(.0049)$ & $2: 2$ \\
\hline $.0023(.0000)$ & & 2.04 \\
\hline $.067 .(.0019)$ & $.0503(.1056)$ & 212 \\
\hline $.00 .32(.0033)$ & $.0604(.0050)$ & $21^{i}+$ \\
\hline
\end{tabular}

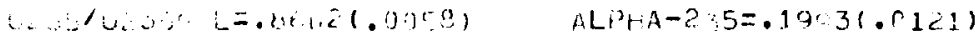

$n+P+i \beta_{A}-230=.31 y(.10,25)$

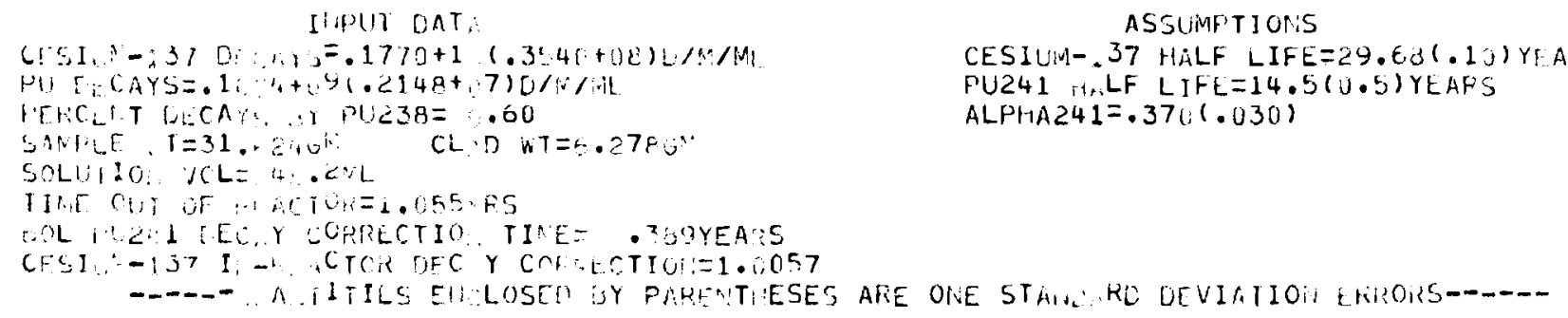




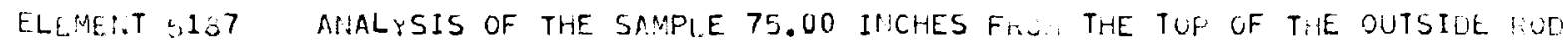

ISOTOPIC I NVENTO,Y

$\begin{array}{ll}\text { ISUTOFL } & \text { TOL }(6) \\ U-234 & .4076-04 \\ U-C 35 & .7076-02 \\ U-236 & .0000 \\ U-C B S & .9781-00 \\ \text { TOTAL } & .9952-00\end{array}$

$\begin{array}{cr}\text { EOL MASS SATIO } & \text { EOL }(A / 0) \\ .5105-04(.237-05) & .0051(.0002) \\ .0091-02(.47-04) & .6646(.0047) \\ .9309-04(.283-05) & .0097(.0003) \\ 1.0(100 & 93.3206(.0047)\end{array}$

EOL. (GN)

$\therefore 011-04(.230-0 b)$

$.0519-02(.471-04)$

$.598-04(.281-05)$

$.914-00(.134-03)$

$:>982-00(.125-03)$

$$
\begin{aligned}
& \text { I.ET PHOOLCTIOL (G) } \\
& .3509-0 t(.23 t-05) \\
& -.5570-03(.4+71-04) \\
& .3593-04(.281-05) \\
& -.1340-02(.124-03) \\
& -.1805-02(.125-03)
\end{aligned}
$$

ISUICFL

$\begin{array}{ll}10230 & 0.03(1.2350) \\ +0640 & 17.0730(.430)\end{array}$

PU241 2.3100(.1060)

i. $)<42$

ISUIOFL

FUas?

$\mathrm{Fu}<40$

Fu<40

Fin

FU⿺42
TUTALS

ISUTOPL

$4-255$

$0-235$

v- 230

Puaje

PUL 41
TUTILS

uess/u3.u. $L=.9213(.0066)$

\begin{abstract}
(ISSIC:S (GM)
$.4003-0 \times(.4723-04)$

$.7959-04(.0360-05)$

$.9(25-0)(.0437-04)$

$.2433-0 \div(.2550-05)$
\end{abstract}

EOL :ASS IATIO

1.0000

$.2589-40(.135-02)$

$.4458-01(.294-0.3)$

$.5230-02(.974-04)$

E. $L(G M)$

$.38 \div 2-02(.3722-04)$

$.99(8-03(.2260-04)$

$.1816-03(.4305-05)$

$.20-4-04(.5072-06)$

$.50: 2-02(.1140-03)$

ALPHA $-2 \pi 5=.2088(.0226)$
EOL $(,>0)$

$76.2767(.812)$

$19.7480(.231)$

$3.5703(.238)$

$.3989(.074)$

¿OL ( $6 \%)$

$.3225-02(.1 \div 29-04)$

. $8275-03(.4,41-05)$

$.1132-03(.0411-04)$

. $1132-03(.13 .41-05)$

$.1130-04(.0105-07)$
$.4777-02(.2+00-04)$

F I 5510 in 5

RUPNIP (MUT/MT:)

442.4

78.0

68.7

$24 \cdot 1$

1513.1

FISSIUN YIELL

$.002(.0014)$

$.002(.0060)$

$.007+(.0019)$

$.000 .0033)$
SPECIFIC ACTIVITY (LIM/GN)

$.1361 b+12(.170+(t 4)$

$.50130+12(.300+10)$

$.57000+10(.100+(0.1)$

$.87000+10(.200+00)$

NET PFOLUCTION (GM)

$.1055-114(.8933-04)$

$.1713-03(.2315-04)$

$.6042-04(.43+3-05)$

$.9041-05(.601,4-1,6)$

$.2653-03(.1165-03)$

INPUT DAT
CESI $1-137$ DEC

DLPHA-239= $.27 \$ 1.15016)$

LEL

$.0491(.0049)$

ME W/FISSION:

$.0563(.0056)$

202

$.0604(.0000)$

214

PU239 FORYEO FROM U.38 $=.1272-02(.13-133)$

ASSLMPTIOIS

CESIUIM- 37 HALF LIFE $=29.68(.10)$ YEARS

PU241 ‥LF LIFE $=14.5(0.5)$ YEARS

ALPHAC4 $=.370(.030)$

PU DLCAYS $=.14 \leq 5+09(.2930+i 7) 0 / M / M L$

PERCENT UECAYS $3 Y$ PUL38 $=7.60$

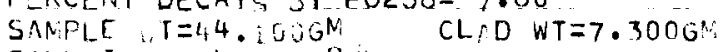

SOLLTIOH VOL $=5.9 \mathrm{ML}$

TIME OUT OF PEACTUR=1.055YRS

DOL FU241 EECAY CORRECTIOU JINE= ZSSYEARS

CESIUR-: 37 I -REACTOR DECIY CORFECTION=1.0057

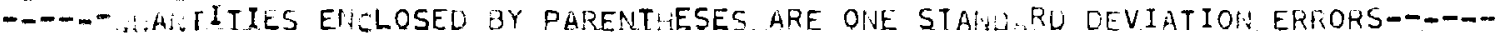


ELEMEIT 1101 _ ANALYSIS OF IHE SAMPLE 85.00 INCHES EROV THE TOP OF THE OUTSIDE ROD

ISOTOPIC INVENTOKY

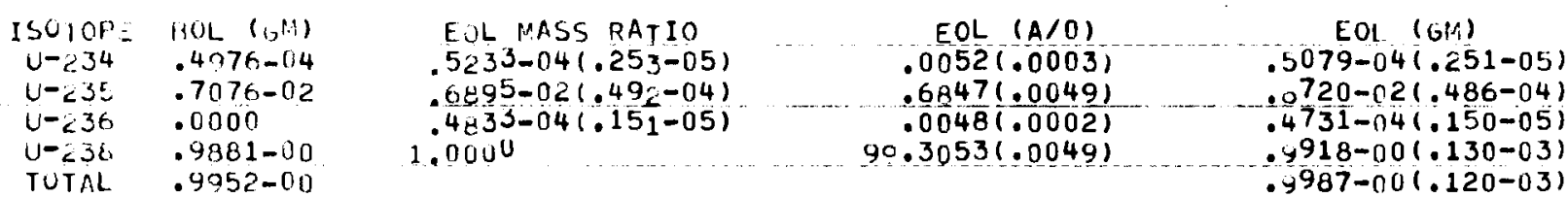

$$
\begin{array}{r}
\text { NET PRODUCTION (GM) } \\
.1027-05(.251-05) \\
-.3559-03(.486-04) \\
.4731-04(.150-05) \\
-.9967-03(.130-03) \\
-.1306-02(.120-03)
\end{array}
$$

\begin{tabular}{|c|c|c|}
\hline $\begin{array}{r}\text { LOL }(A / 0) \\
80.1830(.2(350) \\
17.2730(.0430) \\
2.3100(.1 .060) \\
.2340(.0005)\end{array}$ & $\begin{array}{l}\text { EOL NASS KATIO } \\
1.0000 \\
.2541-00(.846-03) \\
.4007-01(.381-03) \\
.4692-02(.555-04)\end{array}$ & $\begin{array}{r}76.8660(.0556) \\
19.5340(.0527) \\
3.2393(.0304) \\
.3607(.043)\end{array}$ \\
\hline & $\begin{array}{l}\text { EL (GM) } \\
.3800-02(.0729-04) \\
.9851-03(.2228-04) \\
.16(0-03(.4034-05) \\
.18-04-04(.4679-06) \\
.5028-02(.1135-03)\end{array}$ & $\begin{array}{c}\text { BOL (GM) } \\
.3825-02(.1929-04) \\
.8275-03(.4041-05) \\
.1132-03(.0441-06) \\
.1130-04(.6165-07) \\
.4777-02(.2400-04)\end{array}$ \\
\hline
\end{tabular}

F I SSIONS
L. ISSIG:S (GN) . 3, $85,0 \%(.4059-04)$ $.5446,-04(.60831-05)$ $.076 \div-0 \times(.05721-04)$ - $1: 94-04\left(.19^{9} 3-05\right)$ $.159-0 ?(.3157-04)$
BURNUP (NWr/MTM)

$$
\begin{array}{r}
296.2 \\
53.4 \\
667.4
\end{array}
$$$$
\begin{array}{r}
18.8 \\
10.35 .7
\end{array}
$$

FISSION YIELC $.0028(.0014)$ .0620 $.0074(.0019)$
SPECIFIC ACTIVITY $\left(C / M / G M_{i}\right)$ $.13616+12(.170+09)$ $.57600+10(.100+09)$ $.87000+10(.200+00)$

NET PRODUCTION (GNi) $.3503-04(.8940-04)$ $.1576-03(.2276-04)$ $0.503-04(.4085-05)$ $.0036-04(.4085-05)$ $.2505-03(.1160-03)$ $.50130+12(.300+10)$

PUC 41

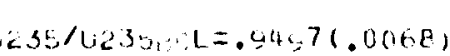

ALPYIA-2 $5=.1525(.0245)$

$\triangle L P H A-239=.346 .3(.218,4)$ ASSLIMPTIONS

INPUT DNTA

CFST U -137 DECAYS $=.6920+031.13811+08) 0 / \mathrm{M} / \mathrm{ML}$

PU LLCAYS =8GCG+081.1791+7)

PERCLIT LECAYS I PU238= .10

SAMPLE :T=29.210CM CL:D WT $=6.03360$ "

SOLUTIOH YOL $=51.1 \mathrm{ML}$

37 HALF LIFE

IFE $=29.68(.10)$ YEARS

PU241 H.LF LIFE $=14.5(0.5)$ YEARS

ALPHA241=.370 $(.030)$

TINE OU OF a ACTOR=1.055)

BOL FUZ4! !EC Y CORPECTIO!! TIME= .389YEARS

CFSIU, 137 I -REACTOR DECAY CORWECTIOR $=1.0057$

- ATITIES ENCLOSED BY PAFENTHESES ARE ONE STANDARD DEVIATION ERRORS---- 
ELENIENT $5: 15$ S ANALYSIS OF THE SAMPLE 2.50 INCHES FRO: THE TOP OF THE CENTER ROD

I 5 O OP I C I NVEN TO I Y

$\begin{array}{cccr}\text { ISUTOPE } & \text { EOL (GM) } & \text { EOL MASS RATIO } & \text { EOL }(A / 0) \\ U-234 & .4976-04 & .5082-04(.221 .05) & .0051(.0002) \\ U-225 & .7076-02 & .5303-02(.350-04) & .5867(.0035) \\ U-236 & .0000 & .2175-03(.48,-05) & .0216(.0005) \\ U-236 & .9381-00 & 1.0000 & 99.3867(.0035)\end{array}$

$$
\begin{gathered}
\text { EOL (GM) } \\
.4929-04(.222-05) \\
.5750-02(.346-04) \\
.2128-03(.481-05) \\
.9912-00(.139-03) \\
.4974-00(.134-03)
\end{gathered}
$$

\begin{tabular}{|c|c|c|}
\hline IsOHE & SOL $(A / C)$ & EOL NASS HATIO \\
\hline$F U_{k} 3 y$ & $00.1830(.6350)$ & 1.0000 \\
\hline$P U_{<} 40$ & $17.2730(.1 .430)$ & $.3244-00(.120-02)$ \\
\hline PU241 & $2.2100(.000)$ & $.6275-01(.42 .2-03)$ \\
\hline$+U_{2}<2$ & .00 & \\
\hline
\end{tabular}

$$
\begin{aligned}
& \text { NET PRODUCTIOH (GM) } \\
& -.4671-06(.222-05) \\
& -.1320-02(.346-04) \\
& .2128-03(.481-05) \\
& -.1515-02(.13(1-03) \\
& -.2632-02(.134-03)
\end{aligned}
$$

\section{$E$ L $(G M)$}

$.3631-02(.8313-04)$

$.1193-02(.2704-04)$

$.2371-03(.5618-05)$

$.3418-04(.8709-06)$
$.5125-02(.1160-03)$

EOL $(A / O)$

\begin{tabular}{|c|c|c|c|}
\hline $\begin{array}{l}\text { ISOTCFE } \\
U-235 \\
U-238 \\
\text { HU } 39 \\
\text { HU4 } \\
\text { TOTALS }\end{array}$ & $\begin{array}{c}\text { FISSIOR }\left(G_{N}\right) \\
.1110-02(.3496-04) \\
.1553-03(.1549-04) \\
.9561-03(.6961-04) \\
.6157-04(.5518-05) \\
.2283-02(.0273-04)\end{array}$ & $\begin{array}{c}\text { BURNUP (MWU/MTN) } \\
1065.4 \\
152.3 \\
042.6 \\
61.1 \\
2221.5\end{array}$ & $\begin{array}{l}\text { FISSION YIELD } \\
.0628(.0014) \\
.0620(.0060) \\
.0674(.0019) \\
.0660(.0033)\end{array}$ \\
\hline
\end{tabular}

$71.5384(.6700)$

$23.2071(.0714)$

$4.5950(.4300)$

$.6596(.076)$

BOL (GM)

$.3825-02(.1429-04)$

$41-05$

$.1132-03(.0441-06)$

$.1130-04(.6165-07)$

$.4777-02(.2400-04)$

F. I $\$$ S I O N $S$

SPECIFIC ACTIVITY $(\mathrm{D} / \mathrm{A} / \mathrm{G}(\mathrm{W})$

$.13616+12(.170+04)$ $.50130+12(.300+10)$ $.57600+10(.100+0)$ $.87000+10(.200+09)$

NET PRODUCTION (G)

$-.1640-03(.8534-04)$

$.3652-03(.2744-04)$

$.1239-03(.5655-05)$

$.2288-04(.8730-06)$

$.3480-03(.1185-03)$
ALPHA $-235=.1945(.0080)$

ALPHA-239 $=.5999(.15 \mathrm{~m} 9)$

$\begin{array}{cc}\text { UEL } & \text { MEV/FISSION } \\ .0636(.0064) & 202 \\ .0826(.0083) & 209 \\ .0855(.0085) & 211 \\ & 214\end{array}$

PU239 FORPED FROM UP38=.1365-02(.14-03)

\section{INPUT DAT}

CESILY-137 DECAYS =.1105+1,1.2210+08) U/M/M4 PU DECAYS $=.7522+08(.1504+7) D / M / M L$

PERCENT DECAYS BY PU238=16.10

SAMPLE,$T=21.200 G M$

SOLUTIOII VOL $=65.2 \mathrm{ML}$

TIME OUT OF REACTOR= 488 TRS

BOL PU241 DECAY CORRECTIO TIME = . 3B9YEARS

CESIUM-137 I I-REACTOR DEC Y CORPECTIOH $=1.0160$

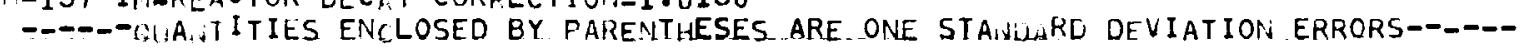

\section{ASSUMPTIONS}

\section{CESI UM-1 32. HALF LIFE $=29.681 .11)$ YEARS}

PU241 HiLF LIFE $=14.5(0.5)$ YEARS

ALPHA241=.370(.030) 
ELEMEIT E1 135 ANALYSIS OF THE SAMPLE 12.50 INCHES FROM THE TOP OF THE CENTER ROD

ISOTOPIC INVENTOKY

$\begin{array}{cc}1 S O T O P E & \text { BOL }(G M) \\ U-234 & .4976-04 \\ U-235 & .7076-02 \\ U-236 & .0000 \\ U-238 & .9881-00 \\ \text { TOTAL } & .9952-00\end{array}$

\begin{tabular}{|c|c|c|}
\hline $\begin{array}{l}\text { EUL MASS RAIIO } \\
.4895-04(.25:-05) \\
.5262-02(.312-04) \\
.3285-03(.560-05) \\
1.0000\end{array}$ & $\begin{array}{r}E Q L(A / O) \\
.0049(.0003) \\
.5232(.0031) \\
.0327(.0006) \\
90.4392(.0031)\end{array}$ & $\begin{array}{c}\text { EOL }(\mathrm{GM}) \\
.4745-04(.252-05) \\
.5122-02(.309-04) \\
.3211-03(.566-05) \\
.9906-00(.156-03) \\
.9962-00(.152-03)\end{array}$ \\
\hline
\end{tabular}

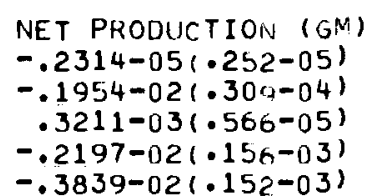

ISOTOFE

PU239

$P U_{240}$

PUC 41

$9952-00$

$.4962-00(.152-03)$

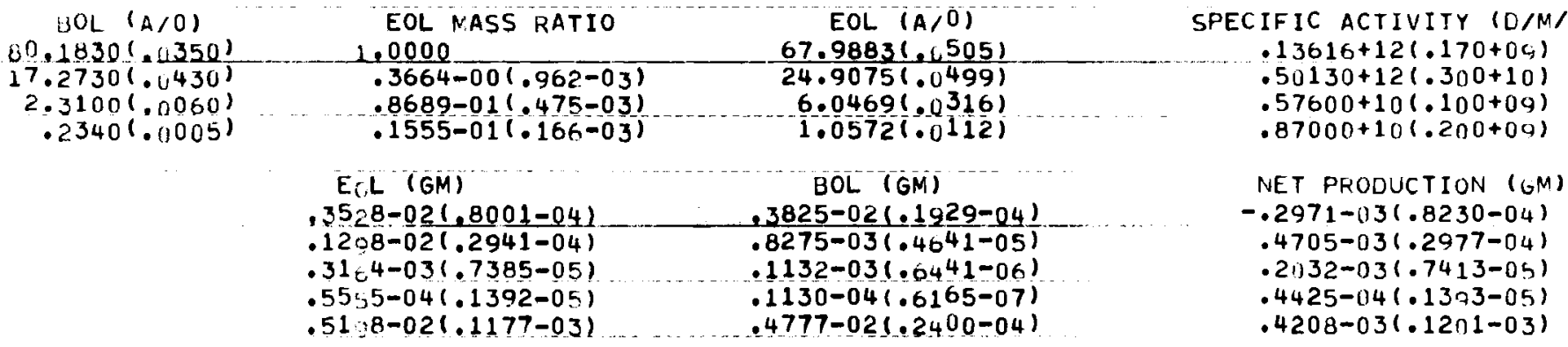

FISSIONS

ISOTOFL
$U-C 35$
$U-238$
PUCSG
PUCA1
TUTALS

FISSISS $(G N)$
$.1025-02(.0141-04)$
$.2326-03(.2295-04)$
$.1434-02(.0540-04)$
$.1191-03(.1036-04)$
$.3410-02(.9365-04)$

EURNOP (MWI/MTM)

1559.5

228,2

1413.9

118.1

$3 \times 19.6$
FISSION YIELD

$.0628(.0014)$

$.0620(.0060)$

$.0674(.0019)$

.060 u $(.0033)$
UEL

$.0636(.0064)$

$.0826(.0083)$

$.0855(.0085)$
MEV/FISSIOIV

202

209

211

214

VC.35/UC35.4L $=.7239(.0043) \quad$ ALPHA $-2.55=.2026(.0057)$

ALPHA-239 $=.583,(.1090)$

PU239 FORMEU FROM U238 $=.1973-02(.15-03)$

$$
\text { INPUT DATA }
$$

CESIUN-137 DESAYS $=.1598+1,(.3196+08)$ D/M /ML

PU PLCAYSE 7 TC

FERCINT LECAYS LYY PU238 14.40

SAMPLE :T=19.382GM CLOD WT $=2.867 \mathrm{GN}$

\section{ASSUMPTIONS}

CESIUM-137. HALF LIFE $=29,68(.10)$ YEAKS
PU24 1 HALF LIFE $=14.5(0.5)$ YEARS

ALPHA241 $=.370(.030)$

TIME OUT OF RFACTURE, 488:RS

SOL PUZW1 CECAY CORRECTIOH TIME= .369YEARS

CESILV-137 I:-REACTOR OEC Y CORPECTION $=1.0160$

---GIAATITIES ENCLOSED BY PARENTHESES ARE ONE STANDARD DEVIATION ERKORS--.-- 
I SOOTOP I C I NVENTO KY

$\begin{array}{cccr}\text { ISOTOPE } & \text { BOL (GI) } & \text { EOL MASS RATIO } & \text { EOL (A/O) } \\ U-234 & .4976-04 & .4751-04(.232-05) & .0047(.0002) \\ U-235 & .7076-02 & .4679-021.294-04) & .4655(.0029) \\ U-236 & .0000 & .4216-03(.650-05) & .0419(.0007) \\ U-236 & .9861-00 & 1.0000 & 99.4878(.0030)\end{array}$

TOTAL $.9952-00$

$\begin{array}{cc}0 O L(A / 0) & \text { EOL NASS RATIO } \\ 10.1830(.0350) & 1.0000 \\ 17.2730(.030) & .4117-00(.122-02) \\ 2.3100(.000) & .1063+00(.546-03) \\ .2340(.0005) & .2216-01(.236-03)\end{array}$

EL (GM)

$.33,3-02(.7688-04)$

$.13 ., 9-02(.3173-04)$

$.36: 7-03(.8592-05)$

$.7591-04(.1904-05)$

$.5227-02(.1185-03)$

EOL. (GM) $.4603-04(.231-05)$ $.4553-02(.291-04)$ $.+120-03(.653-05)$ $. .9901-00(.172-03)$ $.9901-00(.172-03)$
NET PROOUCTION (GM) $-.3733-05(.231-05)$ $-.2524-02(.291-04)$ $.4120-03(.653-05)$ $-.2693-02(.172-03)$ $-.2693-02(.172-03)$
$-.4821-02(.170-03)$
$F U \angle 39$
$P U 240$
$P U 241$
$P U 242$
$15 O T O F$
$P U 239$
$F U 240$
HU241
HU242
TOTALS

\section{F. I SSIONS}

EOL $(A, 0)$

$64.8522(. .574)$

$26.7016(.1 .590)$

$7.0091(.342)$

$1.4371(.151)$

$B O L \quad(G M)$

3825-02(.1929-04)

$.8275-03(.4041-05)$

$.1132-03(.6441-06)$

$.1130-04(.6165-07)$

$.4777-02(.2400-04)$

ISUTOFE

$U-235$

$v-<38$

$P U_{<} 39$

$P \cup 241$

TOTALS

$\begin{array}{cc}\text { FISSIUS }\left(G_{M}\right) & \text { EURNUP }\left(M W_{D} / M T M\right) \\ .2097-02(.<982-04) & 2012.6 \\ .2965-03(.2916-04) & 290.8 \\ .1785-02(.1187-03) & 1759.5 \\ .1739-036.1500-04) & 172.4 \\ .4352-02(.1192-03) & 4235.3\end{array}$

FISSION YIELO

$.0628(.0014)$

$.0621(.0060)$

$.0674(.0019)$

$.4352-02(.1192-03)$

4235.3

$U 235 / U 235 B(1 L=.0434(.0041)$

ALPHA-235=.2037(.0047)

ALPHA-239 $=.597(.0906)$

SPECIFIC ACTIVITY (D/M/GM)

$.13616+12(.170+09)$

$.50130+12(.300+10)$

$.57000+10(.100+04)$

$.87000+10(.200+00)$

NET PROOUCTION (GM)

$-.4420-03(.7926-04)$

$.5713-03(.3207-04)$

$.2555-03(.8616-05)$

$.6461-04(.1905-05)$

$.4495-03(.1209-03)$

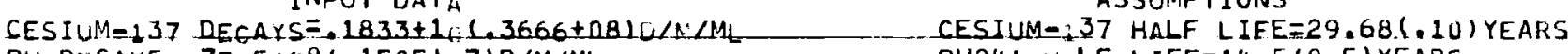

ASSUMPTIONS

PU DECAYS $=.7525+08(.1505+07) D / M / M L \quad$ PU241 HALF LIFE $=14.5(0.5)$ YEARS

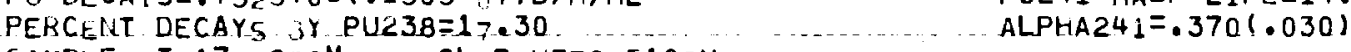

SAMPLE IT $=17.720 \mathrm{GM}$

$C L \perp D$ WT $=2.510 \mathrm{GM}$

SOLUT ION VOL $=256.3 \mathrm{ML}$

TIME OUT OF REACTOR $=.353 Y$ RS

BOL PU241 DECAY CORRECTIOR IIME= .369YEARS

CESI MM- 137 IN-REACTOR DEC AY CORPECTION $=1.0160$

- - GUATITITES ENCLOSED BY PARENTHESES ARE ONE STAND\&RD DEVIATION ERRORS----- 
ELEMENT 5105 ANALYSIS OF THE SAMPIE 33,50 INGHES FROM THE TOP OF IHE CENTER ROD

I S O T O PIC INVENIOH Y

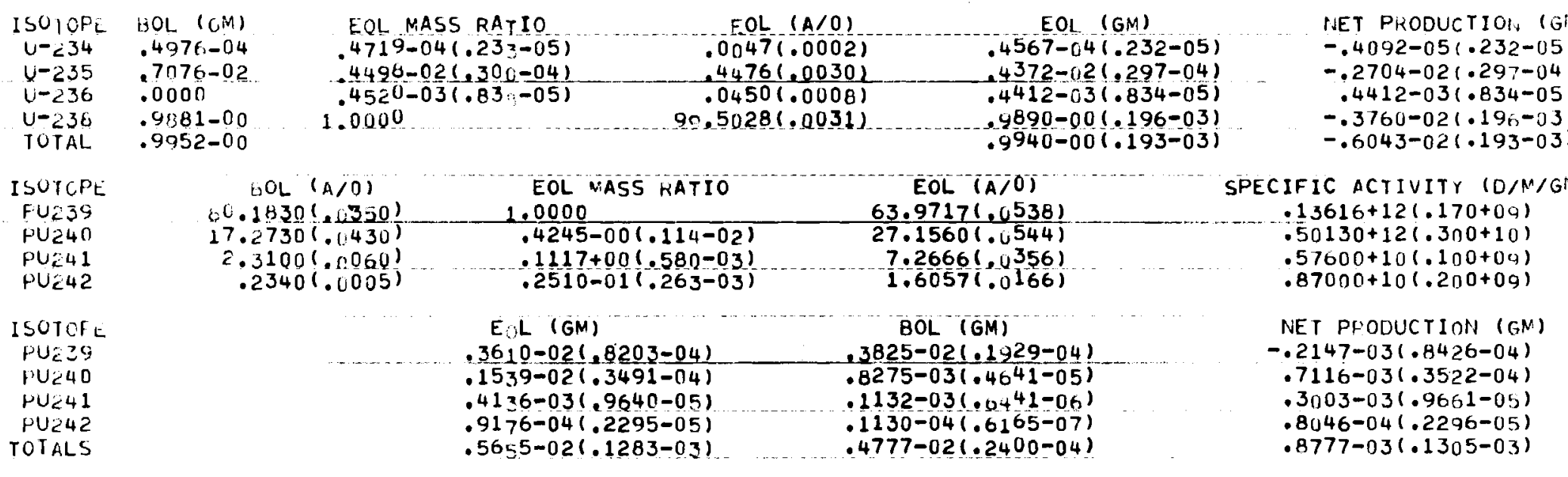

F I S S I O N S

ISUTCFE

$U-<35$

$u-238$

Fucse

Hu<41

FISSIONS (GN)

$.2,45-0 ?\left(\cdot 30^{82}-04\right)$

$.3544-03(.3482-04)$

$.2325-0<(.1406-03)$

- $2325-02(1.1406-03)$

$.2166-03(\cdot 1001-04)$
$.5144-02(.1423-03)$

RURNIJP (MWD $/ M T M)$

2155.4

347,5

2294.8

214.7

5012.4

TOTALS

INPUT DAT A

CESILIV-137 DECAYS $=.2260+1,(, 4520+08)(\mathrm{N} / \mathrm{N} / \mathrm{ML}$

PU) [LCAYS $=.86,1+08(.1732+; 7) \mathrm{D} / \mathrm{M} / \mathrm{ML}$

PERCEIIT DECAYS LY PU238 $=1.70$

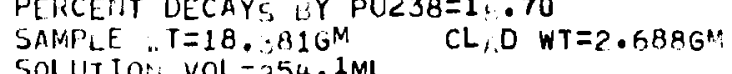

FIME $8 U T$ OF OF ACTORE :353:R5

LOL PU241 OECMY CORRECTIO TIME $=.369$ YEAFS

CESIUN-137 II:-REACTOR DECAY CORFECTION=1.0160

ALPHA-239 $=.5624(.0753)$

FISSION YIELO

$.0628(.0014)$

$.062(.0060)$

.06746 .0014

$.0660(.0033)$

$\begin{array}{cc}\text { DEL } & \text { MEV/FISSION: } \\ .0636(.0064) & 202 \\ .0826(.0083) & 209 \\ .0855(.0085) & 211 \\ & 214\end{array}$

PU239 FORMED FKOM UP38 $=.3420-02(.19-03)$
ASSLMPTIONS

CESIUM-137 HALF LIFE $=29.68(.10)$ YEARS

PU241 HALF LIFE $=14.5(0.5)$ YEARS

ALPHA241=.370 $(.030)$

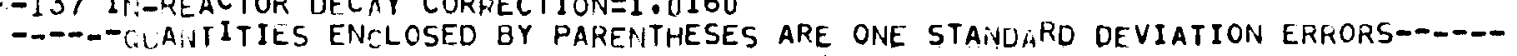


I SOTOPIC I NVENTOKY

\begin{tabular}{|c|c|c|c|c|}
\hline $\begin{array}{l}\text { ISU } 1 C P L \\
U-\angle 34 \\
L-\angle 35 \\
U-236 \\
U-C 36 \\
\text { TUTAL }\end{array}$ & $\begin{array}{l}\text { fol }(0,4) \\
.4776-04 \\
.7076-02 \\
.0000 \\
.9281-00 \\
.9952-100\end{array}$ & $\begin{array}{l}\text { RAYIO } \\
.30:-05) \\
.35(-04) \\
.84-05)\end{array}$ & $\begin{array}{r}E O L \\
.4500-04 \\
.4348-02 \\
.4476-03 \\
.3920-00 \\
.9970-00\end{array}$ & $\begin{array}{l}\text { IVET PRODUCTIOAJ (GM) } \\
-.4758-05(.306-05) \\
-.2728-02(.355-04) \\
.4476-03(.845-05) \\
-.7322-03(.143-03) \\
-.3033-02(.138-03)\end{array}$ \\
\hline $\begin{array}{l}\text { ISUIOPE } \\
P U \subset 39 \\
P U<40 \\
P \cup<41 \\
P U 242\end{array}$ & $\begin{array}{r}101(n / 0) \\
20.1830(.1350) \\
17.0730(\cdot 430) \\
2.310(\cdot 1.050) \\
.2340(.005)\end{array}$ & $\begin{array}{l}\text { EOL IASS RATIO } \\
1.0000 \\
.4289-00(.123-02) \\
.1131+00(.5 A 2-03) \\
.2543-01(.257-03)\end{array}$ & $\begin{array}{r}\text { EOL }(A / 0) \\
63.7205(.0564) \\
27.3291(.0580) \\
7.3301(.0356) \\
1.6204(. .162)\end{array}$ & $\begin{array}{c}\text { SPECIFIC ACTIVITY }(D / M / G M) \\
.13616+12(.170+0(;) \\
.50130+12(.3100+10) \\
.57600+10(.100+0(;) \\
.87000+10(.200+0)\end{array}$ \\
\hline 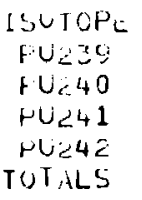 & & $\begin{aligned} & E_{1} L(G M) \\
& .25(8-02(.5837-04) \\
& .1106-02(.2509-04) \\
& .2979-03(.6943-05) \\
& .6612-04(.1645-05) \\
& .4038-02(.9160-04)\end{aligned}$ & $\begin{array}{c}\text { BOL }(G M) \\
.3825-02(.1329-04) \\
.8275-03(.4041-05) \\
.1132-03(.041-06) \\
.1130-04(.0165-07) \\
.4777-02(.2400-04)\end{array}$ & $\begin{array}{l}\text { PET PROUUCTIOII (G,M) } \\
-.1257-02(.6147-04) \\
.2784-03(.2552-04) \\
.1347-03(.6973-05) \\
.5462-04(.1640-05) \\
-.7394-03(.9469-04)\end{array}$ \\
\hline
\end{tabular}

F I S S I. ON S

\begin{tabular}{|c|c|c|c|}
\hline $\begin{array}{l}\text { ISUTUPL } \\
U-\angle 35 \\
U-238 \\
P U \angle 39 \\
\text { PULS } \\
\text { TUTALS }\end{array}$ & $\begin{array}{l}\text { fISSIOI.S (GM) } \\
.2262-02(.3052-04) \\
.2470-0.3(.2444-04) \\
.1080-02(.1040-03) \\
.1475-0.3(.1<76-04) \\
.3737-02(.1009-03)\end{array}$ & $\begin{array}{l}\text { EURNUP }(M W \cap / M T M) \\
2171.5 \\
242.2 \\
1765.1 \\
146.3 \\
3625.2\end{array}$ & $\begin{array}{l}\text { FISSIUN YIELÚ } \\
.0620(.0014) \\
.0626(.0060) \\
.0674(.0019) \\
.065(.0033)\end{array}$ \\
\hline
\end{tabular}

$\begin{array}{cc}\text { DEL } & \text { MEV/FIS5I01! } \\ .0636(.0064) & 202 \\ .0826(.0063) & 209 \\ .0355(.0025) & 211\end{array}$

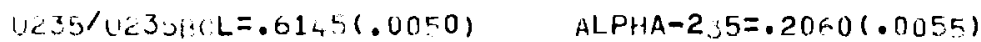

ALPHA-239 $=.6100(.12(7)$

PU239 FORMEU FROM: Uग38 $=.4872-03(.14-0.3)$

INPUT DATA

CESI UH- 37 DECAYS $=.2088+1,(.4176+08) 0 / \mathrm{M} / \mathrm{ML} \ldots$

ASSUMPTIONS

PU DECAYS $=.7914+08(.1583+17) \mathrm{D} / \mathrm{M} / \mathrm{ML}$

PCRCENTT DECAYS UY PU238=1:.30

SAMPLE :T=23.152GM CLAD WT $=3.280 \mathrm{GM}$

CESIUM- 137 HALF LIFE $=29.68(.10)$ YEARS

PU241 HMLF LIFE $=14.5(0.5)$ YEARS

ALPHA241 $=.370(.030)$

$5 O L U T I O H$ VCL $=50.8 M L$

TIME UUT OF RFACTOR= 353 YRS

BOL PU241 DECHY CORRECTIO TIME = . 389 YEARS

CESI UVI:-137 IN

--OHAITITIES ENCLOSED BY PARENTHESES ARE ONE STANO RO DEVIATION ERRORS--.-- 
ELEMEPT 5135 .... ANALYSIS OF THE SAMPLE 44.75 INCHES FROM THE TOP OF THE CENTER ROD

ISOTOPIC INVENTOKY

$\begin{array}{ll}\text { ISOTOPE } & \text { BUL (EN) } \\ U-\angle 34 & .4076-04 \\ U-235 & .7076-02 \\ U-236 & .0000 \\ U-238 & .9581-00 \\ \text { TOTAL } & .9952-00\end{array}$

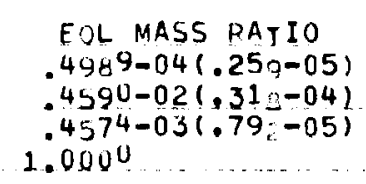

EOL $(G M)$ $.0050(.0003) \quad .4820-04(.257-05)$

$.0455(.0008)$ $.4457-n 3(.788-05)$

$\begin{array}{rr}90.4929(.0033) & 9873-00(.226-03) \\ .9923-00(.224-03)\end{array}$
NET PRODUCTION (GN) $-.1562-05(.257-05)$ $-.2623-02(.315-04)$ $.4457-03(.788-05)$ $-.5467-02(.226-03)$ $-. .7663-02(.224-03)$

ISUICFE

PU.39

PU246
PUC4

PU⿺⿻一𠃋十 242

$100 \mathrm{~L}(A / 0)$ EOL MASS RATIO $63.7562(.0537)$

$17.2730(.0430)$

$2.3100(.0060)$ 1.0000 $.2340(.005)$

$\begin{array}{rr}.4269-00(.113-02) & 27.2150(.0539) \\ .1137+00(.608-03) & 7.3698(.0371)\end{array}$
$.2602-01(.268-03) \cdots$

$.13616+12(.170+09)$ $.50130+12(.300+10)$ $.57600+10(.100+00)$ $.87000+10(.200+09)$

ISCTOPE

PUC39

$\mathrm{PU}_{<} 40$

W $<41$

1.0242

TUTALS

$E L$ (GM) BOL (GM)
$41,9-02(9472-04)$
0

$.4169-02(.9472-04)$

$.17,7-02(.4054-04)$

$.48 \in 0-03(.1134-04)$

$.1098-03(.2739-05)$

$.65(2-02(.1486-03)$

8275-03(1.4-41-0.5)

-8275-03(.4041-05)

$.1130-04(06165-07)$

$.4777-02(.2400-04)$

FISSIONS

I SUTOFt
$\mathrm{U}=35$

$u-235$
$v-238$

$u-238$
$F v=39$

PUत्य 1

TOTALS

\begin{abstract}
1. ISSI $\mathrm{G}_{1} S$ (GM)
. $2160-02(.3248-04)$

$.4116-03(.4044-04)$

. 31313-02(.1633-03)

- $2652-03\left(\cdot 22^{7} 3-04\right.$

$.5176-02(.1001-03)$
\end{abstract}

RURNUP (MWO/MTM)

2073.9

403.6

2706.3

253.0

5736.9
ALPHA-2.:5 $=.2139(.0054)$

ALPHA-239= $=.55 \% 1(.0665)$

INPUT DATA

CESIUM- 237 DECAYS $=.2940+1+1.5880+08) D / M / M L$

PU [LCAYS $=1129+09(.2258+7) \mathrm{D} / \mathrm{M} / \mathrm{ML}$.

FERCLPT DECAYS GY PUZ38 $=1: 00$

SAMPLE WT=0.320GM CL.DWT $=2.9066 \mathrm{M}$

SOLUT IO: VOL $=49.5 \mathrm{ML}$

TIME OUT OF RFACTOR = .353 $Y R S$

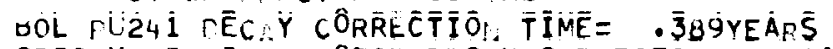

CESILM-137 II:-REACTOK DEC IY COPPECTION:=1.0160

- WATITIES ENCLOSED BY PAREITTHESES ARE ONE STANDARD DEVIATION ERRORS----
FISSION YIELD

FISSION YIELi

$.0620(.0060)$

$.0674(.0019)$

$.0660(.0033)$

ASSUMPTIONS

NNET PRODUCTION (GM)

$.3440-03(.9666-04)$

$.9596-03(.4080-04)$

$.3728-03(.1136-04)$

$.9854-04(.2740-05)$

$.1775-02(.1505-03)$

DEL

$.0636(.11064)$

MEV/F I SSIOR.

.08261 .0083

.08551 .00851

202

211

214

PU239 FORMED FROM U238 $=.5077-02(.22-03)$

CESIUM- 37 HALF LIFE $=29.68(.10)$ YEARS

PU241 HALF LIFE $=14.5(0.5)$ YEARS

ALPHA2 $41=.370(.030)$ 
ELEMEIT F.1.35 ANALYSIS OF THE SAMPIE 54.50 IACHES FiROP THE TOP OF THE CENTER ROD

I S O T O P I C I N VENTO F Y

$\begin{array}{cccc}\text { ISOTOPE } & \text { IOL }(\mathrm{GM}) & \text { EOL MASS PAIIO } & \text { EOL }(\mathrm{A} / 0) \\ U-234 & .4076-04 & .4723-04(.217-05) & .0047(.0002) \\ U-\angle 35 & .7076-02 & .402-02(.347-04) & .4603(.0034) \\ U-236 & .0000 & .4314-03(.83-05) & .0429(.0008) \\ U-238 & .9881-00 & 1.0000 & 90.4020(.0035) \\ \text { TUTHL } & .9952-00 & & \end{array}$

$10 L(A, 0)$

$$
\begin{array}{r}
0.1830(.0350) \\
17.2730(.0430) \\
2.100(.000)
\end{array}
$$$$
.2340(.005)
$$

$$
\begin{aligned}
& \text { EOL VASS RATIO } \\
& 1.0000 \\
& .4118-00(.137-02) \\
& .1081+00(.720-03) \\
& .2319-01(.275-03)
\end{aligned}
$$

$.41,8-02(.9365-04)$

$.176,3-02(.3865-174)$

$.45,7-03(.1083-04)$

$.9670-04(.2485-05)$

$.6374-02(.1446-0.3)$
$E \cdot L$ (GM)

EOL (GM)

$.0566-0.4(.216-05)$ $.4492-02(.344-04)$ $.4206-03(.827-05)$ $.9879-00(.214-03)$ $.9879-00(.214-03)$
$.9930-00(.211-03)$

OL $(4 / 0)$ $64.7249(.062)$ $26.6550(.665)$ $7.1191(.447)$ $1.5010(.178)$

BOL (GM) $.3825-02(.1929-04)$ $.8275-03(.4041-05)$ $.1132-03(.0441-06)$ $.1130-04(.6+65-07)$ $.4777-02(.2400-04)$

F.I S S I Q N 5

$\begin{array}{ll}\text { ISOTOFE } & F I S 5 I 0.5(G M) \\ U-235 & .2147-02(.3538-04) \\ U-238 & .3754-03(.3692-04) \\ \text { PUC39 } & .2647-02(.1498-03) \\ \text { PUEL1 } & .22911-03(.1980-04) \\ \text { TUTALS } & .5399-02(.1511-03)\end{array}$

EURNUP (MWח/MT:
$20 \in 1.3$
368.2
2609.5
227.9
5266.8

IHET PRODUCTIOH: (GM)

$-.4103-05(.216-05)$

$-.2584-02(.344-04)$

$.4206-03(.827-05)$

$-.4829-02(.214-03)$

$-.7010-02(.211-03)$

$23 y / v 23 b 30 \mathrm{~L}=.0348(.0048)$

ALPHA-2:5 $=.2033(.0057)$

ALPHA-239 $=.5797(.0742)$

SPECIFIC ACTIVITY (D/M/G:1)

. $13616+12(.170+09)$

$.50130+12(.3 n 0+16)$

$.57600+10(.100+09)$

$.87000+10(.200+00)$

NET PPODUCTION (GN)

.2929-03(.95o2-04)

.8755-03(.3892-04)

$.3435-0.3(.1034-04)$

$.8539-04(.24\{36-05)$

$.1597-02(.1466-03)$

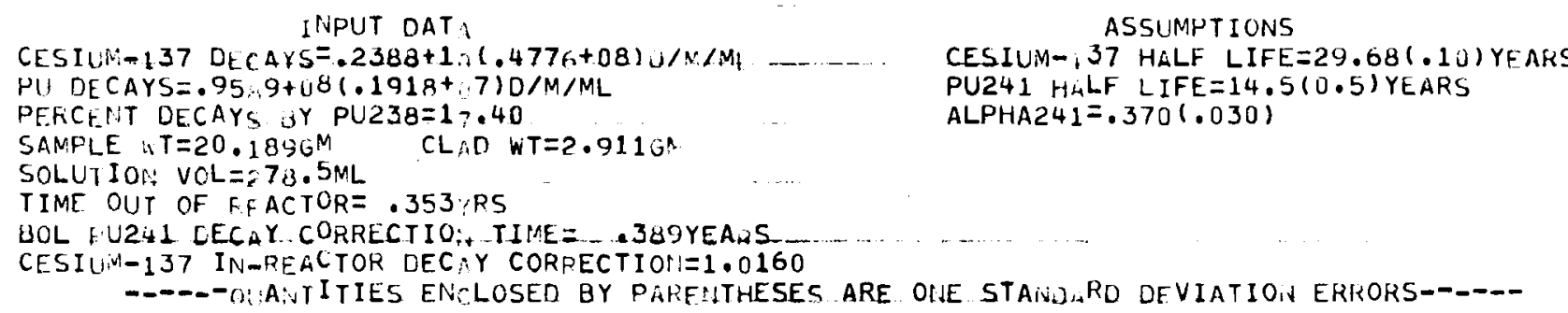


LLEMENT 5185 ALALYSIS OF THE SAMPLE 65.00 INCHES FROM THE IOP OF IHE CENTER ROD

$$
\text { I } 5 \text { O T O P I C I NVENTOKY }
$$

\begin{tabular}{|c|c|c|c|c|c|}
\hline $\begin{array}{l}\text { ISUTCPL } \\
U-\angle 34 \\
U-\angle 35 \\
U-236 \\
U-238 \\
T O T A L\end{array}$ & $\begin{array}{l}\text { HOL }(\mathrm{OM}) \\
.4976-04 \\
.7076-02 \\
.0000 \\
.9881-00 \\
.9952-00\end{array}$ & $\begin{array}{l}\text { EUL NASS RATIO } \\
.4572-04(.21: 05) \\
.4982-02(.313-04) \\
.3674-03(.757-05) \\
1.0000\end{array}$ & $\begin{array}{r}\text { EOL }(A / 0) \\
.0045(.0002) \\
.4055(.0031) \\
.0365(.0008) \\
99.4634(.0032)\end{array}$ & $\begin{array}{c}\text { EOL (GM) } \\
.4437-04(.217-05) \\
.4856-02(.310-04) \\
.3596-03(.753-05) \\
.9918-00(.139-03) \\
.9971-00(.135-03)\end{array}$ & $\begin{array}{l}\text { INET PRODUCTION (GM) } \\
-.5390-05(.217-05) \\
-.2220-02(.310-04) \\
.3596-03(.753-05) \\
-.9866-03(.139-03) \\
-.2860-02(.135-03)\end{array}$ \\
\hline
\end{tabular}

TOTAL $\quad .9952-00$

\begin{tabular}{|c|c|c|}
\hline $\begin{aligned} & 1 O L(A / 0) \\
& 60.1830(.2350) \\
& 17.2730(.0430) \\
& 2.3100(.0060) \\
& .2340(.0005)\end{aligned}$ & $\begin{array}{l}\text { EOL NASS KATIO } \\
1.0000 \\
.3976-00(.364-02) \\
.1016+00(.228-02) \\
.2037-01(.326-03)\end{array}$ & $\begin{array}{c}\text { EOL }(A / 0) \\
65.7333(.1870) \\
26.1356(.1811) \\
6.7921(.1432) \\
1.3390(.0215)\end{array}$ \\
\hline & $\begin{array}{l}E L(G M) \\
.2849-02(.6635-04) \\
.1138-02(.2611-04) \\
.2969-03(.9599-05) \\
.5877-04(.1660-05) \\
.4343-02(.9944-04)\end{array}$ & $\begin{array}{r}\text { BOL (GM) } \\
.3825-02(.1929-04) \\
.8275-03(.4641-05) \\
.1132-03(.0441-06) \\
.1130-04(.6165-07) \\
.4777-02(.2600-04)\end{array}$ \\
\hline
\end{tabular}

3)

SPECIFIC ACTIVITY (O/M/GPi)

$.13616+12(.170+09)$

$.50130+12(.300+10)$

$.57600+10(.100+04)$

$.87000+10(.200+09)$

PU⿺4十

PUट4र

isUTOPt

PU⿺ $\leq 9$

एणत 0

PU⿺41

PU⿺42

$.4343-02(.9944-04)$

$.4777-02(.2 \div 00-04)$

NET PRODUCTION (GM)

-.9757-03(.6910-04)

$.3102-03(.2652-04)$

$.1837-03(.9621-05)$

$.4747-04(.1662-05)$

$-.4343-03(.1023-03)$

F I SSI 0 in $S$

ISO TOF I-

$v-235$

$U-238$

FU 239

Pن 41

(15SIO:S $\left(G N_{1}\right)$

DURNIJP ("WI/MTM)

1775.7

214.4

1063.6
126.7

126.7
3180.4

$.1079-0 ?(.3125-04)$

$.127 \mathrm{~g}-03(.1128-04)$

$.3275-02(.8000-04)$

FISSION YIELI)

$.0628(.0014)$

$.0624(.0060)$

$.0674(.0019)$

$.060(.0033)$

$.0636(.0064)$

MEV/FISSIOI:

$.0826(.0083)$

$.0855(.0085)$

202

209

211

U235/LC3 J1.CL $=.0 R(2(.0044)$

ALPHA $-2,25=.2003(.0059)$

ALPHA $-239=.6203(.1263)$

PU239 FORIAED FFOM U238 $=.7712-03(.14-0.3)$

INPUT DAT

CESI JP-137 DECAYS $=.2013+1](.4026+08) 0 / \mathrm{M} / \mathrm{ML}$

ASSUMPTIONS

PU CE CAYS $=.893+U 8(.1785+17) 0 / M / A L$

PERCENT DECAYS LY PU238=1.70

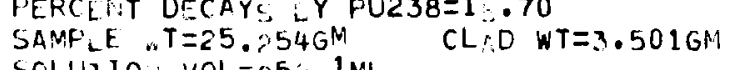

TIME OUT OF RACTOR= .353;RS

GOL HI241 IDEC YY CORRECTIOP: TIME= -389YEARS

CESILIM-137 II-REACTOK DEC AY CORRECTION=1.0160

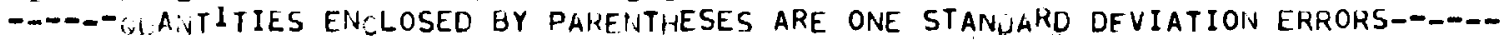

作

PU241 HALF LIFE $=14.5(0.5)$ YEARS

ALPHA24 $1=.370(.030)$ 
ELEMMTT i.: ANAL YSIS OF THE SNIPLE 2.50 INCHES FFO, THE TOP UF THE MIDCLE ROO

$$
\text { IS O TOP I C I NVENTOUY }
$$

\begin{tabular}{|c|c|c|c|}
\hline $\begin{array}{l}I S U 1 C F \\
U-\angle 34 \\
U-232 \\
U-\pi 30\end{array}$ & $\begin{array}{l}10 \mathrm{~L}(0) 1) \\
.4976-04 \\
.7076-02 \\
.0700 \\
.9881-00\end{array}$ & $\begin{array}{l}\text { EUL MASS PATIO } \\
.4348-04(.22,-05) \\
.5708-02(.426-04) \\
.2 .72-03(.51 ;-05) \\
1.0000\end{array}$ & $\begin{array}{c}E O L(A / D) \\
.0049(.0002) \\
.5733(.0042) \\
.0336(.0005) \\
90.3482(.0042)\end{array}$ \\
\hline
\end{tabular}

(.OL (in) $0.1830(.2350)$ $17.7730(.430)$ 2. $200(.0000)$ $.340(\cdot 0,0,15)$
EOL I ASS :ATIO 1.0000

$.4275-00(.127-02)$

$.4275-00(.127-02)$
$.11 ? 9+00(.723-03)$

.2633-01(.310-03)

E L (GM)

. $3130-02(.7115-04)$

$.13,3-02(.3049-114)$

$.3643-03(.8607-05)$

$.03 .4-04(.2136-05)$

49 1-021.1117-03)

$$
\text { EUL }(6: 1)
$$

$4000-04(.228-05)$

$.619-1.2(.420-04)$

- $321-(13(.514-05)$

$.9914-00(.139-03)$

. 9974-001.132-03)
EOL $(A / 0)$

$63.7251(.010)$

$27.2412(.605)$

$27.2412(.0605)$
$7.3558(.444)$

$1.6779(.195)$

$50 L(G M)$

$. \triangle 825-02\left(.1\right.$ y $\left.<9-0 t_{i}\right)$

$.8275-03(.4041-05)$

$.1132-03(.0441-06)$

$.1130-04(.0165-07)$

$.4777-02(.2400-04)$
F I S I ONS

LSC1OPL

$v-35$

r.u. 39

r.U.41

TUTALS

\begin{abstract}
IISIO:S $\left(G_{i,}\right)$
- $1221-02(.4227-04)$

$.1594-03(.1601-04)$

- $906-0 \times(.7670-04)$

$.11 .42-03(.1076-04)$

$.2477-02(0.078-04)$
\end{abstract}

FURNUP (VWIMTM:)
1171.7
156.3
989.9
132.5
2410.5

FISSION YIELO
$.0620(.0014)$
$.002(.0060)$
$.0674(.0019)$
$.000 .0033)$

DEL

$.0609(.0061)$

HET PFODUCTIO:, (GW

$-.1761-05(.220-05)$

$-.1457-02(.420-04)$

$.2321-03\left(.514_{4}-05\right)$

$-.1393-02(.134-03)$
$-.2623-02(.132-03)$

$235 / U_{c} 35, L=.7942(.0059)$ ALPHA-2:5=.1934(. กU83)

AL PHA-239=1.1440(.1316)

$.0766(.0077)$

$.0799(.0080)$

MEV/FISSIOH

202

209

211

214

PU239 FORMEO FROM U>33 $=.1239-02(.14-i) 3)$

INPUT DAT:

CESIL.H-137 DLCAYS $=.8670+0 G(.1774+08)[: \mathrm{M} / \mathrm{ML}$

PU] CECAYS $=.550 .0+u(.1120+7) \mathrm{D} / \mathrm{M} / \mathrm{ML}$

PERCENT DECAYS IY PU23\% 1.20

ASSUMPTIONS

CESIUIH- 37 HALF LIFE $=29.68(.10)$ YFARS

PU241 HNLF LIFE $=14.5(0.5)$ YEARS

ALPHA241 $=.370(.030)$

SAMIPLE: $T=15,27 \mathrm{MM}$

$C L \cdot D W T=0.7420$

SOLUTIO: VOL $=54.4 \mathrm{ML}$

TIME OUT OF OACTUR= .458,RS

EOL WU241 CEC.Y CORRECTIO TIME = . 3U9YEA:S

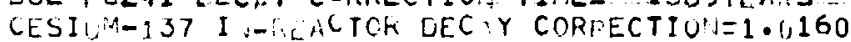

- - OA. ITIES ENICLOSED GY PARFNTHESES ARE ONE STA'VLIRD DEVIATION LKFORS-- 
ELEMEIII S125 ANALYSIS OF IHE SAMPLE 12,50 INCHES FROM THE TOP OF JHE MIODLE ROD

\section{SOTOP I C I NVENTOKY}

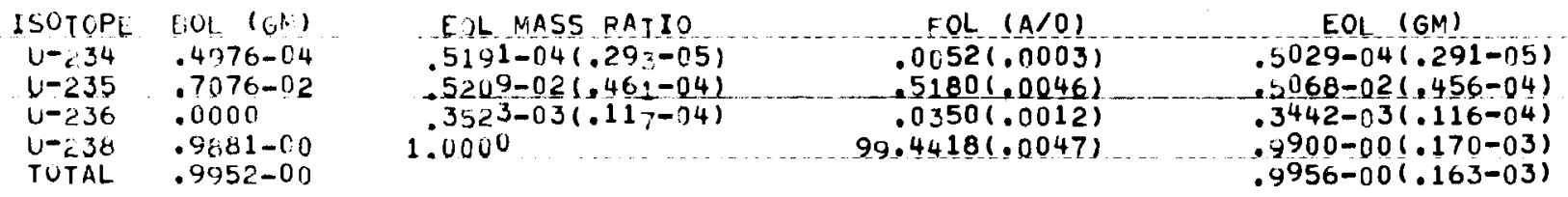

NET PRODUCTIOH, (GM) $.5276-06(.291-05)$ $-.2008-02(.456-04)$ $.3442-03(.116-04)$ $-.2744-02(.170-03)$ $-.4418-02(.163-03)$

\section{ISOTOPE \\ PU:39 \\ $\mathrm{Fu}_{2} 40$ \\ Pण241 \\ I SOTOPE. \\ Pui 39 \\ FU< 40 \\ Pli241 \\ गण242 \\ TCIALS}

BOL $(A / 0)$

EOL MASS RATIO

EOL $(A>0)$

SPECIFIC ACTIVITY (D/M/GM)

$\frac{30.1830(.1350)}{17.2730(.143 j)}$

$2.3100(.000)$

1.0000

$67.1352(.0689)$

$6.2551(.0348)$

$1.1883(.0275)$

$.13616+12(.170+09)$

$.50130+12(.300+10)$

$.57000+10(.100+00)$

$.87000+10(.200+09)$

$\begin{array}{rr}E L(G M) & B O L(G M) \\ .3624-02(.8238-04) & .3825-02(.1929-04) \\ .1378-02(.3126-04) & .8275-03(.4641-05) \\ .3405-03(.7991-05) & .1132-03(.6441-06) \\ .6405-04(.2120-05) & .1130-04(.6165-07) \\ .5497-02(.1226-03) & .4777-02(.2400-04)\end{array}$

F I 5.5 I $0 \mathrm{~N} 5$

ISOTOPL FISSIUSS (GM)

$u-\angle 35$

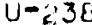

+ 4039

PLC41

TOTALS
$.1055-02(.4705-04)$

$\cdot 2457-03(\cdot<440-04)$

$.1735-02(.1114-03)$

$.1444-036.1303-04)$

$.3780-02(.1047-03)$
BURNUP (MWU/MTH)

1589.1

241.0

1710.5

143.2
3683.7

U235/U230EUL $=.71 \div 2(.0064) \quad$ ALPHA-2:5=.2131(.0103) INPUT OAT:

CESILM-137 DECAYS $=.1495+1(, 2990+081 \mathrm{~L} / \mathrm{M} / \mathrm{ML}$ PII DLCAYS $=.63_{C} 4+06(.1385+.7) 0 / \mathrm{M} / \mathrm{ML}$

PERCELT DECAYS LY PU238 $=14.40$

SAMPLE T=16.924GM CL:D WT=2.5926

FISS ION YIELO

$.0620(.0014)$

$.0621(.0060)$

$.0674(.0019)$

$.0600(.0233)$
DEL

$.0609(.0061)$

$.0766(.0077)$

$.0799(.0080)$
NET PRODUCTION (GN)

$-.2011-03(.8461-04)$ $.5506-03(.3160-04)$ $.2273-03(.8017-05)$

(3)-04(.2121-05)

PU239 FORMED FROM U2 38 $=.2508-02(.17-03)$

MEV/FISSIOI

202
209
211
214

AL PHA-239= $.56 \% 31.09451$

TINE OUT OF TIACTOR=.276\%RS

BOL WU241 LECAY CORRECTIOI: TINE= .389YEAFS

CESIUM-137 IN-REACTOR DEC,Y CORRECTION=1.0160

- WANTIIES ENCLOSED GY PARENTHESES ARE ONE STANDARD DEVIATION ERRORS-----

CESIUM- 37 HALF LIFE $=29.68(.10)$ YEARS

PU241 HLF LIFE $=14.5(0.5)$ YEARS

ALPHA241 $=.370(.030)$

\section{ASSIJMPTIONS}


ELEMEIT :165 ANAL, SIS OF THE SAMPLE 23.50 INCHES FROM. THE TCP OF THE MIDLLE RCO

$I \leq 0$ TOPIC INVENTO:Y

$\begin{array}{cccc}\text { ISOTCFL } & \text { EOL (UD) } & \text { EUL NASS RATIO } & \text { FOL }(A / 0) \\ U-234 & .4976-04 & .5460-04(.41-05) & .0054(.0004) \\ U-235 & .7076-02 & .4513-02(.44-04) & .4490(.0044) \\ U-230 & .0000 & .4469-03(.121-04) & .0445(.0012) \\ U-C 30 & .9881-00 & 1.0600 & 90.5011(.0046)\end{array}$

TCTAL $98,81-00$

$.9952-\mathrm{CO}$

$90.5011(.0046)$

\begin{tabular}{|c|c|}
\hline & \\
\hline ISUTOFE & $\therefore O L \quad(\therefore / 0)$ \\
\hline$P U<39$ & $10 \cdot 1830(.2350)$ \\
\hline $\begin{array}{l}+U_{c} 40 \\
P U_{<41}\end{array}$ & $\begin{array}{r}17.2730(.430) \\
2.3100(.060)\end{array}$ \\
\hline
\end{tabular}

$$
\begin{aligned}
& \text { EOL "ASS ISATIO } \\
& 1.0000 \\
& .1+246-00(.147-02) \\
& .1124+00(.755-03) \\
& .2524-01(.32 \pi-03)
\end{aligned}
$$

E. L (GM)

35. $9-02(.8167-04)$

$.1531-02\left(.3475-0 l_{t}\right)$

$.41,1-03(.9781-05)$

$.91>1-04(.2395-05)$

$.5623-02(.1276-03)$

F I S.S. I N S

IsUTOF:

$v-\angle 35$

$(1-23 \varepsilon$

Pu. 39

PUC 41

TOTALS
RURNUP (NWO/MTM)

2145.8
321.2
2232.0
214.6
4913.6

EOL (GM) $.5285-i 4(.410-05)$ $.4387-02(.445-04)$ $.4363-03(.120-04)$ .4363-03(.120-041) $.9941-00(.190-03)$
NET PRRODUCTIOH $(\mathrm{G}: 4)$ $.3085-05(.410-05)$ $-.2689-02(.445-04)$ $.4363-13(.120-04)$ $-.3638-02(.19 k,-03)$ -.5911-02(.190-03)

\footnotetext{
LEStUC3StSL $=.0199(.0063) \quad$ ALPHA-2 $35=.2031(.0076)$
} INPUT DATA

CESI (U. 137 DFCAYS $=.2078+1,(.4156+08) U / M / M L$ PU DECAYS $=.79 \cdot .4+U 8(.1599+7) \mathrm{D} / \mathrm{M} / \mathrm{ML}$ PERCENT DECAYS OY PU238 $=1.00$ SAMPLE T=17.

SOLUTIOI, VOL $=57.6 \mathrm{ML}$

TIMF OUT OF RIACTUR= .276YRS

BOL WU241 LECAY CORRECTID

CESIL'M-137 I:-REACTOR DEC Y CORTECTIO!I=1.0160

- WIATITIES ENCLOSED BY PARENTHESES ARE ONE STAWUARE DEVIATION ERRORS--.--

EOL $(A, 0)$ $63.9414(.0092$ $27.1623(.6701)$

$7.2825(.0461)$

$1.6139(.6204)$

BOL (GM)

$.3825-02(.1429-04)$

$.8275-03(.4041-05)$

$.1132-03(.6441-06)$

$.1130-04(.0155-07)$

4777-02(.2400-04)

(1)

$.0674(.0019)$

$.0664(.0033)$

\section{SPECIFIC ACTIVITY ([ / / M/GM) $.13616+12(.170+00)$ $.50130+12(.3 n 0+10)$ $.57600+10(.100+00)$ $.87000+10(.200+09)$}

$\begin{array}{cc}\text { DEL } & \text { MEW/FISSIOI: } \\ .0609(.0061) & 212 \\ .0760(.0077) & 209 \\ .0799(.0080) & 211 \\ & 214\end{array}$

PU239 FOR:AL FROM UP38 $=.3325-02(.19-03)$

\section{ASSUMPTIONS}

CESIUM- 137 HALF LIFE $=29.681 .10)$ YEARS

PU241 H.MLF LIFE $=14.5(0.5)$ YEARS

ALPHA241 $=.370(.030)$

.07601 .00

209

11 
ELEMEUT S13S ANALYSIS OF THE SAMPLE 33.50 INCHES FROH THE TOP OF THE MIODLE RCD

I SOTOPIC IN VEN NORY

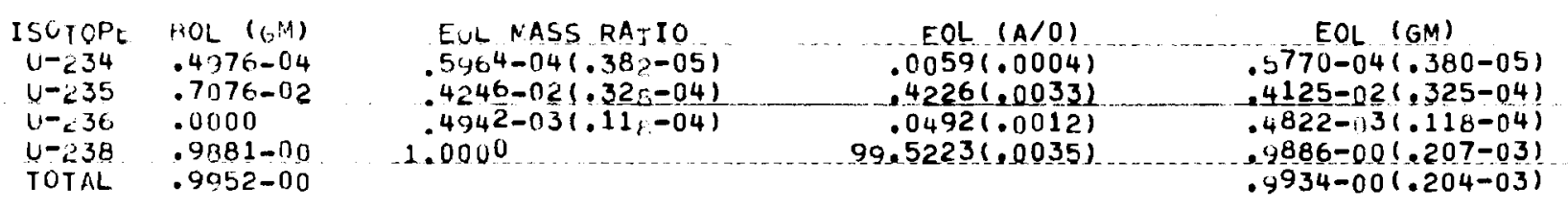
NET PRODUCTION (GM) $.7934-05(.380-05)$ (.) $-.2951-02(\cdot 325-04)$
$-.4130-02(.207-03)$
$-.6623-02(.204-03)$

ISOTOPE.

PUC30

$\mathrm{PU}_{<40}$

PU $<41$

$P \cup<42$

$\therefore O L(A / O)$

EOL MASS RATIO

$E O L(A / 0)$

SPECIFIC ACTIVITY (D/M/GM)

$80.1830(\cdot[350)$

$2.3100(.0430$ 10000

$62.6145(.0555)$

$13616+12(.170+09)$

$.1200+00(.638-03) \quad 7.6123(.0380)$

$1.8227(.0190)$

$.50130+12(.300+10)$

$.87000+10(.200+04)$

ISOTCPL

PU: 39

FU⿺ 40

PUट 41

10242

TOTALS

E $L(G M)$

BOL (GM)

$.3622-02(.0234-04) \quad .3825-02(.1929-04)$

$.1623-02(.3684-04) \quad .8275-03(.4641-05)$

$.4440-03(.1037-04) \quad .1132-03(.0441-06)$

$\begin{array}{ll}.10,8-03(.2676-05) & .1130-04(.6165-07) \\ .57 .6-02(.1315-03) & .4777-02(.2400-04)\end{array}$

F I S I I N 5

\begin{tabular}{|c|c|c|c|c|c|}
\hline $\begin{array}{l}\text { ISUTCPL } \\
U-225 \\
U-238 \\
\text { PUO39 } \\
\text { PUCLI } \\
\text { TUTALS }\end{array}$ & $\begin{array}{c}\text { FISSIU.S }\left(G N_{1}\right) \\
.2446-0 ;(.3460-04) \\
.3624-03(.3570-04) \\
.2509-02(.1529-03) \\
.2569-0:(.2204-04) \\
.5575-02(.1540-03)\end{array}$ & $\begin{array}{c}\text { BURNLP }\left(M W_{C} / M T M\right) \\
2348.4 \\
355.4 \\
2474.1 \\
254.8 \\
5432.7\end{array}$ & $\begin{array}{l}\text { FISS1ON YIELD } \\
.0628(.0014) \\
.0624(.0060) \\
.0674(.0019) \\
.066(.0033)\end{array}$ & $\begin{array}{c}\text { OEL } \\
.0609(.0061) \\
.0766(.0077) \\
.0799(.0080)\end{array}$ & $\begin{array}{l}\text { MEV/FISSION } \\
202 \\
209 \\
211 \\
214\end{array}$ \\
\hline
\end{tabular}

U235/U2351.1L $=.5830(.00(146) \quad$ ALPHA-235=.2062(.0064)

$\triangle L P H A-239=.5894(.0731)$

PU239 FORMED FROM Uح3B $=.3783-02(.20-03)$

INPUT DATA

CESIUN-137 DECAYS $=.2320+1 .(.4640+08)(\mathrm{M} / \mathrm{M} / \mathrm{ML}$

PIJ OECAYS $=.8515+08(.1703+1,7) 0 / \mathrm{M} / \mathrm{ML}$

PEKCENT UECAYS LY PU238=1,10

SAMPLE :T=17.:16GM CLAD WT=2.482.GM

ASSUMPTIONS

SOLIITI IOI VCL $=57.5 M L$

NET PRODUCTION (GM)

$-.2035-03(.8457-04)$

$.7960-03(.3714-04)$

$.3308-03(.1039-04)$

$.9545-04(.2676-05)$

$.1019-02(.1337-03)$

TIME OUT OF REACTOR=.276.RS

B̈OL PUIZ4 I TE E

CESIUN-137 I -REACTOR DEC YY CORPECTION=1.0160

-

CESIUM- 37 HALF LIFE $=29.68(.10)$ YEARS

PU241 HALF LIFE $=14.5(0.5)$ YEARS

ALPHA241 $=.370(.030)$ 
ELEMENT, 135 AIJALYSIS OF THE SA:PLE 37.50 INCHES FRU:. THE TOH OF THE MIODLE RQD

I 5 OTOP I C I NVENTOFY

$\begin{array}{lllr}\text { ISOTOPE } & \text { BOL (GM) } & \text { EOL MASS RA IIO } & \text { EOL (A/O) } \\ U-\angle 34 & .4976-04 & .5265-04(.337-05) & .0052(.0003) \\ U-235 & .7076-02 & .4259-02(.333-04) & .4239(.0033) \\ U-236 & .0000 & .4934-03(.121-04) & .0491(.0012) \\ U-\angle 36 & .9881-00 & 1.0000 & 90.5218(.0035)\end{array}$

$$
\begin{aligned}
& E O L(G M) \\
& .0093-04(.335-05) \\
& .4138-02(.330-04) \\
& .4814-03(.120-04) \\
& .9866-00(.208-03) \\
& .9934-00(.205-03)
\end{aligned}
$$

HET PRODUCTION (GM) $.1171-05(.335-05)$ $-.2938-02(.330-04)$ $.4814-03(.120-04)$ $-.4150-02(.201-03)$

$-.4150-02(.20)-03)$

\begin{abstract}
$1 \mathrm{OL}(A / 0)$
$00.1830(.0350)$

$17.2730(.430)$

$2.3100(.1060)$

$.0340(.0005)$
\end{abstract}
EOL NASS RATIO
1.0000
$.4481-00(.189-02)$
$.1203+00(.635-03)$
$.2940-01(.315-03)$

\section{$E C L$ (GM)}

ISOTOPE

Fن 39

Pिट40

PU 241

PU242

TOTALS

$$
\begin{aligned}
& \text { ISOTGPE } \\
& U-\angle 35 \\
& U-238 \\
& \text { PUC39 } \\
& \text { PU } 241 \\
& \text { TOTALS }
\end{aligned}
$$

EOL $(A / 0)$

$62.5247(.0791)$

\begin{tabular}{|c|c|c|}
\hline $\begin{aligned} & \text { FISSIOS }(G M) \\
& . 2435-02(.3509-04) \\
& . 3647-03(.3593-04) \\
& . 2548-02(.1540-03) \\
& .2583-03(.2217-04) \\
& . 5605-02(.1550-03)\end{aligned}$ & $\begin{array}{c}\text { BURNUP }(M W \cap / M T M) \\
2337.3 \\
357.2 \\
2511.8 \\
256.1 \\
5462.9\end{array}$ & $\begin{array}{l}\text { FISSLON YIELD } \\
.0620(.0014) \\
.0020(.0060) \\
.0674(.0019) \\
.0660(.0033)\end{array}$ \\
\hline
\end{tabular}

$28.0173(.0860)$

$7.6197(.384)$

$1.8382(.194)$

BOL (GM)

$-3825-02(.1929-04)$

8275-031.46

$\begin{array}{ll}.4428-03(.1037-04) & .1132-03(.0441-06) \\ .1073-03(.2703-05) & .1130-04(.6165-07) \\ .5775-02(.1312-03) & .4777-02(.2400-04)\end{array}$

$\begin{array}{ll}.4428-03(.1037-04) & .1132-03(.0441-06) \\ .1073-03(.2703-05) & .1130-04(.6165-07) \\ .5775-02(.1312-03) & .4777-02(.2400-04)\end{array}$

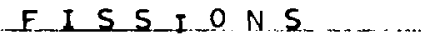

$4235 / \cup 233 H C L=.5848(.0046) \quad$ ALPHA $-235=.2068(.0066)$

4) $\mathrm{PHA}-239=.58011(.0717)$
SPECIFIC ACIIVITY (D/M/GM)

$.13616+12(.170+09)$ $.50130+12(.300+10)$ $.57600+10(.100+00)$ $.87000+10(.200+00)$

NET PRODUCTION (GM) $-.2219-03(.8447-04)$ $.7939-03(.3714-04)$ $.3296-03(.1039-04)$ $.9596-04(.2704-05)$ $.9975-03(.1334-03)$

DEL

$.0609(.0061)$

$.0766(.0077)$ $.0799(.0080)$

202
209
211
214

\section{INPUT DATA}

CESI LM $=137$. DFCAYS $=.2550+1 A\left(.5100+0810 \angle M \angle M_{4}\right.$ PU DECAYS $=.9304+08(.1861+07) 0 / \mathrm{M} / \mathrm{ML}$

PERCENT DECAYS BY PUL38=1 9.30

SAMPLE $W T=20.698 \mathrm{GM}$

SOLUTIOH; VOL $=277.8 \mathrm{ML}$

TINE OUT OF RFACTOR= .276\%RS

UOL PU241. DECAY CORRECTIQW IIMEE . 389YEAOS

CESIUM-137 II:-REACTOR DECAY CORPECTION=1.0160

---- 1 A 4 ITIES ENCLOSEO BY PARENTHESES ARE ONE STAINUARD DEVIATION ERRORS-----

\section{ASSUMPTIONS}

CESIUM -137 HALF LIFE $=29.68(.10)$ YEARS

PU241 HALF LIFE $=14.5(0.5)$ YEARS

ALPHA24 $1=.370(.030)$ 
ELEMENT 5185 ANALYSIS OF THE SAMPLE 3R,00 INCHES FROM THE TOP OF THE MIDDLE ROD

I SOTOPIC INVENTOAY

$\begin{array}{ll}\text { ISOTCPE } & \text { EOL }(G N) \\ U-334 & .4076-04 \\ 1-235 & .7076-02 \\ U-236 & .0000 \\ U-\angle 3 E & .9831-00 \\ \text { TOTAL } & .9952-00\end{array}$

\begin{tabular}{|c|c|c|}
\hline $\begin{array}{l}\text { EOL NASS RATIO } \\
.4770-04(.207-05) \\
.4277-02(.261-04) \\
.4973-03(.753-05) \\
.0000\end{array}$ & $\begin{array}{r}\text { KOL }(A / O) \\
.0047(.0002) \\
.4256(.0026) \\
.0495(.0007) \\
99.5201(.0027)\end{array}$ & $\begin{array}{c}\text { EOL }(G M) \\
.4645-04(.202-05) \\
.4183-02(.259-04) \\
.4684-03(.749-05) \\
.9952-00(.961-04) \\
.1000+01(.922-04)\end{array}$ \\
\hline
\end{tabular}
NET PRODUCTION (GM) -.3309-05(.202-05) $-.2893-02(.259-04)$ $.4884-03(.749-05)$ $.2439-02(.961-04)$ $.2439-02(.961-04)$

TOTAL

$.9952-00$

$.1000+01(.922-04)$

ISU1OPL
$P U 239$
$P \cup C 40$
$P U 241$
$P U \angle 42$

ISOTOPE

PU⿻3

$r U_{<}<0$

PU241

1.0242

TOTALS

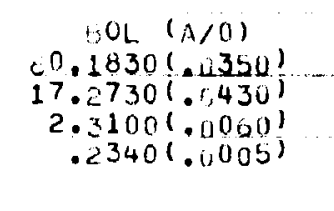

EOL NASS RATIO EOL $(A ̈ / 0)$

$1.0000-\frac{62.6855(.6529)}{28.0029(.0529)}$

$.1129+00(.573-03) \quad 7.5095(.0385)$

$1.8022(.0181)$

$E, L(G M)$

$1345-02(\cdot 3058-04)$

$.6035-03(.1370-04)$

$.16,5-03(.3799-05)$
$.39+7-04(.9755-06)$

$.2151-02(.4880-04)$

BOL (GM)

$.3825-02(.1929-04)$

$.1132-03(.06441-06)$

$.1130-04(.6165-07)$
$.4777-02(.2400-04)$

F I S S I $0 N S$

ISUIGPL

$U-2 J 5$

$U-\therefore 38$

Pण239

$+0241$

TOTAL.
PURNIIP (MWD/MTM)

$$
\begin{array}{r}
2288.1 \\
146.4 \\
-46.8 \\
74.4
\end{array}
$$$$
2462.0
$$

FISSION YIELD
$.0628(.0014)$
$.0620(.0060)$
$.0674(.0019)$
$.0604(.0033)$

SPECIFIC ACTIVITY (G/M/GM)

$.13616+12(.170+09)$ $.50130+12(.300+10)$ $.57600+10(.100+09)$ $.87000+10(.200+09)$

NET PPODUCTION (GM) $-.2480-02(.3615-04)$ $-.2239-03(.1446-04)$

$.4931-04(.3853-05)$

$.2786-04(.9774-06)$

$-.2627-02(.5438-04)$

$\begin{array}{cc}\text { DEL } & \text { ME } / F I S S I O N \\ .0609(.0061) & 202 \\ .0766(.0077) & 209 \\ .0799(.0080) & 211 \\ & 214\end{array}$

UC35/U23 JAd. $=.5911(.0036) \quad$ ALPHA-2.35=.2138(.0045)

$$
\text { INPUT DATA. }
$$

CESIUN-137 DECAYS $=.1055+1(.2110+08) D / M / M L$

PU DECAYS $=.34 .7+08(.6814+.6) \mathrm{D} / \mathrm{M} / \mathrm{ML}$

PERCEIT DECAYS OY PUZ38 $=2,90$

SAMPLE WT=17..259GM

$C L A D W T=2.480 G M$

TIME. OUT OF REACTOR=1.244:RS

BOL FU241 DECAY CORRECTIO:: TIME= .389YEAHS

CESIUM-137 IN-REACTOR DECAY CORPECTION=1 0160

- - QUANTITIES ENCLOSED BY PARENTHESES ARE ONE STTANDARD DEVIATION ERRORS-----

\section{ASSUMPTIONS}

CESIUM -137 HALF LIFE $=29.681 .101$ YEARS

PU241 HALF LIFE $=14.5(0.5)$ YEARS

ALPHA241 $=.370(.030)$ 
ELEMET.T 1. S MNAL SSIS CF THE SAPLE 44.75 INCHES FRU. THL TUP OF THE MIUDLE RQL

I $5 O T O P I C$ I N V E N T O \& Y

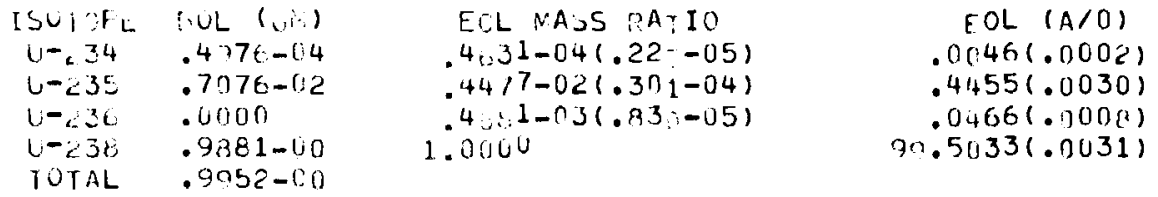

ISUTUF' UOL $(1, / 6)$

$\mathrm{Fu} 239$

Hes?

PU⿻

1041
$+U 242$

ISOTUFi

PUट3?

PU:40

$P U=41$

Fiv 40

TUTALS

$$
\begin{aligned}
& \text { EOL VASS KATIO } \\
& 1.0000 \\
& .3361-00(.147-02) \\
& .5573-01(.474-03) \\
& .1035-01(.124-03)
\end{aligned}
$$

EL ( $(N M)$

$.23 \div 8-03(.5652-05)$

$.3658-04(.9470-06)$

$.4942-02(.1120-13)$
$.1178-02(.2674-04)$
$.34,00-02(.7937-0(1)$

$$
\begin{aligned}
&E O L(6) i) \\
& .4488-04(.226-05) \\
& .4358-02(.298-04) \\
& .4576-(3(.826-05) \\
& .4904-00(.169-03) \\
& .4954-00(.166-03)
\end{aligned}
$$

EOL $(A / 0)$

$70.7348(. .779)$

\begin{tabular}{|c|c|c|}
\hline SSUTCPL & $F \operatorname{IgSI}, S(G, M)$ & I.URNIP $\quad$ U/MT \\
\hline$u-25$ & $\cdot 2,43-0,(.3095-04)$ & 2152.9 \\
\hline$v-238$ & $.2511-03(.2771-04)$ & 275.7 \\
\hline$P U_{c}=39$ & $\cdot 1: 0.3-0 \%(.1198-03)$ & 1778.0 \\
\hline$\because 2 \leq 41$ & -0, $03-041.5079-05)$ & \\
\hline TUTAL & $.4395-02(.1208-03)$ & 4274.0 \\
\hline
\end{tabular}

$23.7740(. .799)$

$4.7591(.333)$

$.7321(.090)$

BOL (GM)

.3825-02(.1,29-04)

$.6275-03(.4041-05)$

$.1132-03(.0441-06)$

$.1130-04(.0165-07)$

$.4777-02(.2400-04)$
F I $5 \mathrm{~S}$ I $0 \mathrm{NS}$

FIS2, ON YIELD)
$.0626(.0014)$
$.0621 .0060)$
$.067 .(.0019)$
$.0600(.0033)$

CFSIL -137 DE CAYS $=.1480+1.2960+0 B) 0 / \mathrm{M} / \mathrm{ML}$ PU RLCAYS $=.54 \div 4+1) 8(.1115+17) 0 / \mathrm{M} / \mathrm{ML}$

PERCENT DECAYG, GY PUL38 $=1 ; .90$

SAMFLE $T=15.249 \mathrm{GM}^{\mathrm{M}} \quad \mathrm{CL} D \mathrm{D}$ WT=?.713 $\mathrm{G}$

$5 O L U T I O:$ VOL $=63.5 \mathrm{ML}$

TIME GUT OF AC ACIONE .48E.RS

LOL FU24 1 DEC, Y CORRECTIO: TINE= .309YEALS

CESIL $"-137$ I:-RLACTOK DFC Y CORPECTION=1.0160

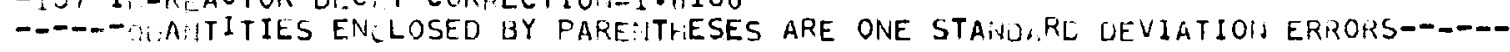

ASSIMPTIONS
CESIUIM- 37 HRLF LIFE $=29.68(.10)$ YEAKS

PU24 1 , LF LIFE $=14.5(0.5)$ YEAP?

ALPHAC41=.370(.030)
SPECIFIC ACTIVITY (U/A/GM)

$\begin{array}{cc}\text { DEL } & \text { MAE } W / F I S S I G i 1 \\ .0609(.0061) & 202 \\ .0766(.0077) & 209 \\ .0799(.0080) & 211 \\ & 214\end{array}$

$.0799(.0080)$

214

$$
\begin{aligned}
& \text { CCTIOH: (uit } \\
& 5(.22 n-05) \\
& \text { (.29k-04) } \\
& 4570-03(.22(-0)-0) \\
& 02(.16 \cdot 1-0.3) \\
& -.4589-02(.160)-03)
\end{aligned}
$$

\section{PU239 FOR:AED FRON U238 $=.2035-02(.17-03)$}


ELEMENT 5145 ... ANALYSIS OF THE SAMPLE 45.25 INCHES FROE THE TOP OF THE MIDDLE ROD

ISOTOPIC INVENTORY

$\begin{array}{ll}\text { ISUTOPL } & \text { 13OL (GIi) } \\ U-234 & .4976-04 \\ U-635 & .7076-02 \\ U-236 & .0000 \\ U-C 38 & .9881-00 \\ \text { TOTAL } & .9052-00\end{array}$

EUL MASS PATIO
$.4300-04(.200-05)$
$.4402-02(.281-04)$
$.4684-03(.78 ;-05)$
1.0000

EOL $(A / 0)$ $.0049(.0002)$ $.4460(.0028)$ $.0466(.0008)$ $99.5026(.0029)$

EOL $($ GM)
$.4738-(14(.199-05)$
$.470-02(.279-04)$
$.4587-03(.778-05)$
$.9922-00(.140-03)$
$.9972-00(.137-03)$

EOL $(A / 0)$ $301 \quad(A / 0)$

Put39

$\mathrm{PU}_{2} 40$

PU:41

PUit' $60.1830(.2350)$
$17.2730(.1 .430)$
$2.3100(.0 .060)$ $.2340(.0005)$

\begin{abstract}
EOL MASS RATIO
\end{abstract} 1.0000

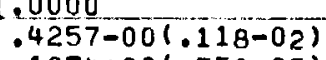

$.1074+00(.550-03)$

$.2578-01(.261-03)$

$63.8778(.2556)$

$27.1947(.0562)$

$7.2808(.0377)$

I SUTCP=

$\mathrm{PU}_{<} 39$

Pن $>40$

$\mathrm{PU}_{6} 4$

PU⿺4⿻一𠃋十

TC, T H LS

\section{$E$ L (GM)}

$.22-5-02(-5147-04)$

$.96 .4-03(.2197-04)$

$.2603-03(.6087-05)$

$.59 ; 3-04(.1470-05)$

$.35,3-02(.8059-04)$

BOL (GM)

.3825-025.1929-04) $.8275-03(.4 \mathrm{i} 41-05)$ $.1132-03(.0441-06)$ $.1130-04(.6165-07)$ $.4777-02(.2400-04)$

\section{F. I S S I O IN}

EURNIIP ("WR/MTN)

2139.8
246.3
1339.6
127.6
3053.4
. $2529-02(0095-04)$
IHET PHODUCTIOH (GN)

$-.2379-05(.190-05)$

$-.2706-02(.27(3-04)$

$.4587-03(.77(-05)$

$-.5123-03(.140-03)$

$-.2779-02(.137-03)$
IS, GTOFC

$1,-35$

$4-238$

$\mathrm{PU} \times 3 \mathrm{G}$

$P U_{6}+1$

TCTILS

FISS1ON YIELD
$.0620(.0014)$
$.062(.0060)$
$.0674(.0019)$
$.0660(.0033)$
$.2512-03(.2477-04)$ - $1259-02(.1676-03)$ $.3968-02(.1476-03)$ $3 \cap 53.4$

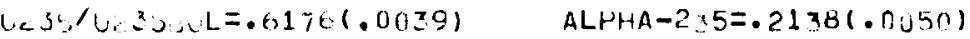
INPUT DATA

CESI $N-137$ DECAYS $=.1025+1,(.3250+08) U / \mathrm{M} / \mathrm{ML}$

PU RECAYS $=.54: 5+08(.1081+.7) 0 / \mathrm{M} / \mathrm{ML}$

PERCE.HT DECAYS, BY PU2 $38=21.60$

$\triangle A M P L E \therefore T=17.147 G M \quad C L D W T=2.594 \mathrm{GM}$

SOLUT IOI: VOL $=46.1 \mathrm{ML}$

TIME OUT OF RI ACTOR=1.244.RS

BOL PU241 EECAY CORPECTIO! TINE= .389YEARS

CESIU" -137 IN-REACTOK DECAY COREECTION=1.0160

1) PHA-239= $.341+(.0792)$

ASSUMPTIONS

CESIUM- 37 HALF LIFE $=29.68(.10)$ YEARS

PU241 HALF LIFE $=14.5(0.5)$ YEARS

ALPHA241 $=.370(.030)$
ECIFIC NCTIVITY (L/M/GM $.13616+12(.170+09)$ $50130+12(\cdot 300+10)$ $.87000+10(.200+09)$

NET PRODUCTION (GM) $-.1560-02(.5497-04)$

$.1409-03(.2245-04)$

$.1471-03(.6121-05)$

$.4782-04(.1472-05)$
$-.1224-02(.8409-04)$

$\begin{array}{cc}\text { DEL } & \text { MEV/FISSIOH } \\ .0609(.0061) & 202 \\ .0766(.0077) & 209 \\ .0799(.0080) & 211 \\ & 214\end{array}$

PU239 FORIVE! FHON U238=.2622-03(.14-03) 
ELMERT IHS MINAL, SIS OF THE. SAMPLE 54.50 INCHES FrUI. THE TUP OF THE MIDULE ROD

$$
\text { I SO TOP I C I N }
$$

\begin{tabular}{|c|c|c|c|}
\hline \multirow{2}{*}{\multicolumn{4}{|c|}{ EL MASS RATIO }} \\
\hline & & & $5 \mathrm{OL} \quad(A / O)$ \\
\hline$u-<35$ & $.71076-02$ & $4,0^{2}-0,(.27-04)$ & $.4242(.0023)$ \\
\hline-2.30 & . Groo & $.405-03(.10 n-04)$ & $.0478(.0010)$ \\
\hline$-<36$ & $.9081-00$ & $1.0,0$ & $90.5232(.0029)$ \\
\hline
\end{tabular}

$1071 \mathrm{~L} \quad .968 .1-60$

1.0000

90.52321

$$
\begin{array}{cc}
\text { EOL (GM) } & \text { IET PROOUCTIOH! (GM) } \\
.4652-04(.298-05) & -.3244-05(.29 m-15) \\
.4120-02(.276-04) & -.2956-02(.27 n-04) \\
.4664-03(.996-05) & -.4664-03(.996-05) \\
.4836-00(.305-03) & -.9158-02(.305-03) \\
.4883-00(.304-03) & -.1167-01(.304-03)
\end{array}
$$

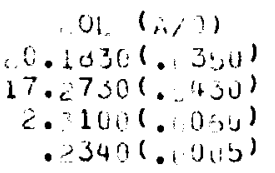

(.) $(2,1)$

$0.1030(.30,0)$

$17.2750(.450)$

c. $100(.050)$

$.2340(.0005)$

EOL NASS PATIO

1.0000

$.4452-00(.330-02)$

$1195+00(.114-02)$

$.2937-01(.552-03)$

$$
\text { E- }-\mathrm{L}(\mathrm{GM})
$$

51. 5-02(.1201-(.3)

$.23,8-02(.5290-04)$

$.63 \cdot 7-03(.1602-06)$

$.15 t 2-03(.4596-05)$

$.8207-02(.1894-0 x)$

$$
\begin{gathered}
\text { EOL }(A / U) \\
62.6205(.1424) \\
27.8786(.1549) \\
7.6617(.069) \\
1.8392(.0342) \\
\text { BOL }(G M) \\
.3825-02(.1929-04) \\
.8275-03(.4041-05) \\
.1132-03(.0441-06) \\
.1130-04(.6165-07) \\
.4777-02(.2400-04)
\end{gathered}
$$

F I S S I O N 5
Fis I 5 (GN) $.2467-02(.2432-04)$ $.5445-03(.5364-04)$ $.474: 3-0 ;(.2097-03)$ . $340-0,3(.205-04)$ $.0145-02(.2562-03)$
PURNUP (Ur; /MTN)

$$
\begin{array}{r}
2368.6 \\
534.0 \\
4681.6 \\
781.4 \\
7965.5
\end{array}
$$

SPECIF IC ACTIVITY (OUN/GN) $.13616+12(.170+09)$ $.50130+12(.300+10)$ $.57000+10(.1110+09)$ $.87000+10(.200+09)$

$$
\begin{aligned}
& \text { INET PROLUCTION (GM) } \\
& .1300-02(.1216-(\mathrm{G}) \\
& .1491-02(.5318-04) \\
& .5265-03(.1003-04) \\
& .1429-03(.4597-0.05) \\
& .3520-02(.1910-0.3)
\end{aligned}
$$

FISSION YIELD
$.0620(.0014)$
$.0620(.0060)$
$.007+(.0019)$
$.0604(.0033)$

LEL

$\begin{array}{cc}\text { LEL } & \text { MEV/FISSION } \\ .0609(.0061) & 202 \\ & 209 \\ .0766(.0077) & 211 \\ .0799(.0080) & 214\end{array}$

PU239 FORIAEL FFOM U238 $=.8650-02(.30-0.3)$

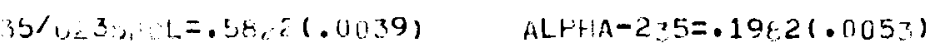

ALPHA-239= $.535(.0547)$

\section{ASSUMPTIONS}

CESIUM- 37 HALF LIFE $=29.681 .10)$ YEARS

PU2 41 H. HF LIFE $=14.5(0.5)$ YEARS

ALPHA $241=.370(.030)$

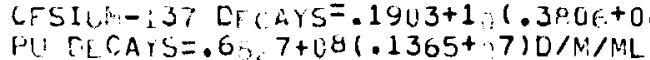

PERCLITT DECAYS IYY PUZ38=1C,20

SAMFLE IT $T=10.1000 \mathrm{M}$

$C L: D W T=2 \cdot 115 \mathrm{GW}$

TINE OUT OF $\because A C T O R=.488$ RS

BOL WU2:1 LECHY CORRECTION TIME= 389YEANS

CESIL, 137 I - 24CTCK DEC Y CORFECTIOU $=1.0160$

-.. A.JITIES ENCLOSED BY PAREITHESES ARE ONE STANGKRD DFVIATION EFRORS--.-- 
ELEMENT : 145 ... ANALYSIS OF THF SAMPLE 55,00 INCHES FRO IHE TOP OF THE MIDDLE ROD

I S O T O P I C I N V E N T O H Y

$\begin{array}{ll}\text { ISOIOPL } & \text { BOL (GN) } \\ U=-34 & .4976-04 \\ U-\angle 35 & .7076-02 \\ U-\angle 36 & .0000 \\ U-\angle 3 E & .9881-00 \\ \text { TUTAL } & .9052-00\end{array}$

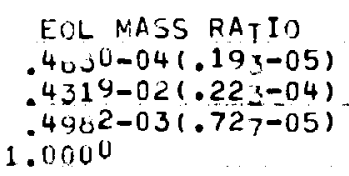

$E O L(A / O)$

$\begin{array}{ll}.0046(.0002) & .4506-04(.192-05) \\ .4298(.0022) & .4221-(.2(.221-04) \\ .0496(.0007) & .4890-03(.723-05)\end{array}$

$99.5160(.0023)$

ISOTCPE $\quad \mathrm{OL}(A / 0)$

PU:39 $\quad 0.1830(.1350)$

$P U 240 \quad 17.2730(.430)$

2. $3100(.0060)$

$.340(.1005)$

PU 242

¿SOTOF

Huceg

$F U_{c}: 40$

小241

riviti

TOTHLS

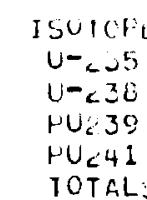

\section{EOL MASS RATIO}

1. 0000

$.4461-00(.110-02)$

$.1145+00(.601-03)$

$.2935-01(.303-03)$

ERL (GM)

$.1560-02(.3547-04)$

$.69(1-03(.1587-04)$

$.1913-03(.4479-05)$

$.4638-04(.1158-05)$

$.247-02(.5667-04)$

EOL $(A / 0)$
$62.6160(.0550)$
$27.9355(.0551)$
$7.6108(.0401)$
$1.8378(.0187)$

BOL (GM)

.3825-02(et929-04)

$.8275-03(.4641-05)$

$.1132-03(.6441-06)$

FISSIONS

TIURNIP (YWO/MT:A)

2751.6
168.5
256.1
93.6
2769.9

2769.9
$.4777-02(.2400-04)$

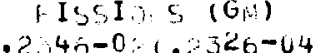

$.1719-021.1711-04)$

- $2: 94-0)(.3058-04)$

$.3440-04(.3606-05)$
-2 $72-02(.3009-04)$

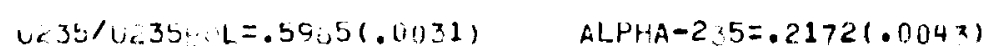

$$
\text { INPUT DATA }
$$

CESIUI -137 DELAYS $=, 1893+1,(3780+08) 0 / M / M L$

PU DECAYS $=.6217+08(.1249+7) \mathrm{D} / \mathrm{M} / \mathrm{ML}$

PERCEIUT LECAYS TYY PUZ3B=2!.50

SAMPLE OT=27. 1 . $20 \mathrm{OM}^{\mathrm{M}} \mathrm{CL}, \mathrm{D}$ WT $=3.8096 \mathrm{M}$

SOLUIIOII VOL $=44.7 \mathrm{ML}$

TIME OUT OF $\triangle$ ICTOR=1.244YRS

GOL PUZW1 CEC Y CURRECTIO TIME= 38GYEARS

CESILUI-137 IN-FEACTOR DEC AY CORPECTION $=1.0160$

-.--ALTITIES ENCLOSED BY FARENTHESES ARE ONE STANDGRD DEVIATION ERRORS-----

ALPHA-239= $.304 .0(.3004)$

$$
\text { ASSUMFTIONS }
$$

CESIUM- 37 HALF LIFE $=29.68(.10)$ Y

ALPHA2 $41=.370(.030)$
$. .4221-(22(.221-04)$

$.4890-03(.723-05)$

$.9945-00(.104-03)$

$.9994-00(.101-03)$

$.1130-04(.6165-07)$
FIS5, ON YIELC

.002 is $(.0014)$

$.0620(.0060)$

$.0674(.0019)$

$.0661(.0033)$
NET PRODUCTION $\left(G v^{*}\right)$ $-.4704-05(.192-05)$ $-.2855-02(.221-04)$ 4890-03(.723-05) $.1746-02(.104-03)$ $-.6421-03(.101-03)$

SPECIFIC ACTIVIIY (D/M/GM)

$.13616+12(.170+4 G)$

$.50130+12(.300+10)$

$.57600+10(.100+09)$

$.87000+10(.200+00)$

INET PRODUCTION (GM)

-.2265-02(.4038-04)

-.1233-03(.1653-04)

$.7805-04(.4525-05)$

$.3507-04(.1159-05)$

$-.2280-02(.6154-04)$

$\begin{array}{cc}\text { DEL } & \text { MEV/FISSIOIJ } \\ .0609(.0061) & 202 \\ .0766(.0077) & 209 \\ .0799(.0080) & 211 \\ & 214\end{array}$

PU239 FORINED FROM U>38 $=-.1920-02(.10-03)$

CESIUM- 37 HALF LIFE $=29.68(.10)$ YEARS 
ELLMEHT :1:S AMALISIS OF THE SNMPIE 64.50 INCHES FRO. THE TOP OF THE MILLLE ROU

$$
\text { I } 5 O T O P \text { I C I HVENTU,Y }
$$

\begin{tabular}{|c|c|c|}
\hline & & \\
\hline ISUDCL & UL (G!) & EUL WASS FATIO \\
\hline $1-.34$ & $.4 c 7 t-14$ & $.4-41-04(.23-05)$ \\
\hline$u-235$ & $.7070-02$ & $.431^{4}-02(.321-04)$ \\
\hline $0-236$ & 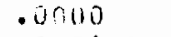 & $.4 \cup 0^{8}-03(.90-05)$ \\
\hline$(-\leq x\}$ & $.92,81-150$ & 1.0000 \\
\hline TUTAL & $.9052-0 n$ & \\
\hline
\end{tabular}

is,

$H=0 \quad 20.1830(.300)$

He 40

IVutition

F U 442

ISUTCF'

ru<3?

Pن 40

Pن241

HC: 4 ?

TUTALS

ISUTCFL

ij- 435

$u-230$

$+U_{c} 39$

PUCLI

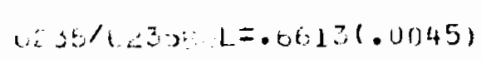

\begin{abstract}
FISSI ( 5 (GN)
$1+87-0,2(\cdot 2<98-04)$

-

10 $3-03(01231-04)$

$.1093-03(.1031-04)$
$.4602-02(.1295-03)$
\end{abstract}

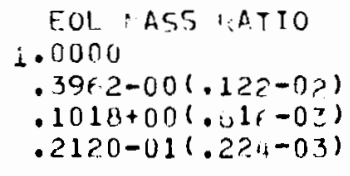

E.OL:ASS I:ATIO i. 0000

$.3962-00(.122-0 ?)$

$.1018+00(.016-0 z)$

$.2120-01(.224-03)$

F. $\mathrm{L}(\mathrm{GM})$

$.38,3-02(.8640-04)$

$.15: 3-02(.3431-04)$
$.39(7-03(.9400-05)$

$.3907-03(.9400-05)$
$.814-04(.2045-05)$

$.87 .7-02(.1314-0 ?)$ $\pm O L(A / O)$

$.4789(.0032)$

$.0405(.0009)$
$.0046(.0002)$
EOL (UA) $.449 z-4(.236-05)$ $.4679-12(.317-1) 4)$ $.0971-1,36.894-(15)$ $.9691-00(.189-03)$
$.994-00(.186-03)$

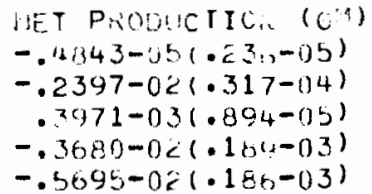

SPECIFIC ACTIVITY (T./M/GM)

$.13616+12(.170+0.3)$

- $50130+12(.3,10+1,1)$

$.50130+12(.3,0+10)$
$.570(00+10(.100+0.7)$

$.57000+11(.100+0.1)$
$.87000+10(.200+09)$

$65.7210(.0002)$

$26.0367(.004)$

$1.3933(\ldots 146)$

BOL (Givi)

$.3825-0 c\left(.16 \cos ^{\prime} 9-04\right)$

$.0275-03(0.4+1-05)$

$1132-03(0.441-00)$

. $1132-03(.0441-06)$

$.1130-04(.0165-07)$
$.4777-02(.2400-04)$

F I S5IONS

FURHIP (MNIN/MT:S)

1007.0

297.9

297.9
2151.7

2151.7
187.7

4544.3

FISS: ON YIELE

$.062,(.0014)$

$.00<$ (.0060)

$.0674(.0019)$

$.0001 .0033)$

$\therefore$ PHA-230 $=.50+3(.0310)$

IET PKOQUCTLA (GM)

$-.2198-04\left(.8553-()_{4}\right)$

$.6050-93(.3463-(14)$

$.2804-03(.9422-012)$

$.7034-041.2016-05)$

$.1020-02(.1336-0.3)$

$\begin{array}{cc}\text { JEL } & M E: / F I S S I O H \\ .0609(.0001) & 202 \\ .0760(.0077) & 209 \\ .0799(.0080) & 211 \\ & 214\end{array}$

PU239 FOR ES FROI: $11238=.3391-02\left(.1^{\circ}-0.3\right)$

CESILI- 37 DFCAY $=.2243+1-(.4486+08) \mathrm{O} / \mathrm{M} / \mathrm{ML}$

HU COCAYS $=.93(1)+08(.1970+7) \mathrm{D} / \mathrm{N} / \mathrm{ML}$

HERCLIT DECAY Y PUE $38=1.50$

SAMPLE :T=20.:19OW CLOD WT=2.877心

SCLUIION VOL= $54.4 \mathrm{ML}$

$488, \mathrm{CL}$

EOL U241 CEC, Y CORFECTIO!. TIME= 3E9YEA

CESIUN-137 I - MCTOR UEC:Y CORHECTIOH:1.0160

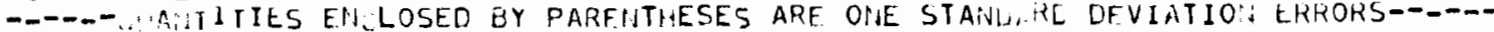

ASSINMIRTIONS

CESIUIM- 37 HALF LIFE $=29.08(.1$ U) YEARS

PU241 H LF LIFE $=14.5(0.5)$ YEARS

ALPhA241 $=.370(.030)$ 
ELEMENT 5185 .. ANAL YSTS OF THE SAMPLE 65.00 INCHES FRQH_THE TOP OF THE MIODLE ROD

I SOTOPIC IN VENTOFY

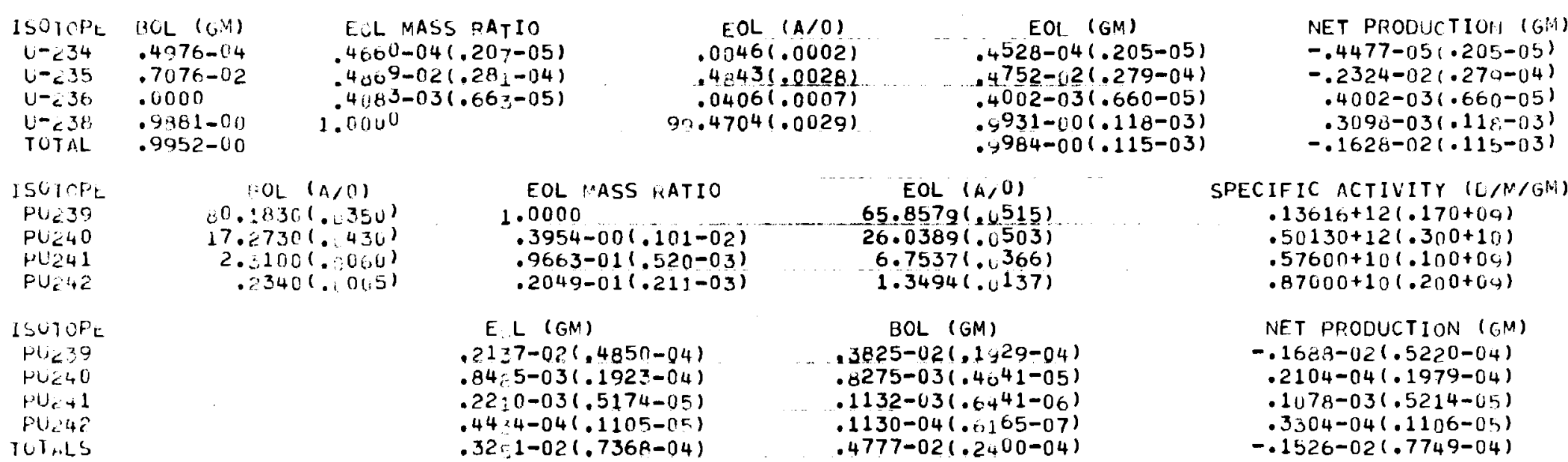

F I S 5 I $O N S$

\begin{tabular}{|c|c|c|c|c|c|}
\hline $\begin{array}{l}\text { ISOICPL } \\
U-C 35 \\
U-C 38 \\
P U_{C}=0 \\
V U_{C} 41\end{array}$ & 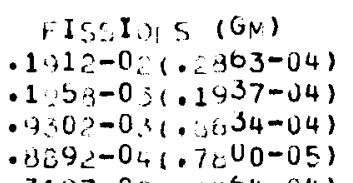 & $\begin{array}{c}\text { FURNUP }(\text { AWr/MTM) } \\
1935.4 \\
192.0 \\
r 17.1 \\
88.2 \\
7\end{array}$ & $\begin{array}{l}\text { FISSION YIELO } \\
.0623(.0014) \\
.0620(.0060) \\
.0674(.0019) \\
.0664(.0033)\end{array}$ & $\begin{array}{c}\text { OEL } \\
.0609(.0061) \\
.0766(.0077) \\
.0799(.0080)\end{array}$ & $\begin{array}{l}\text { MEV/FISSIOI } \\
202 \\
209 \\
211 \\
214\end{array}$ \\
\hline
\end{tabular}

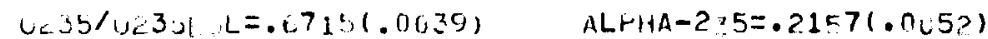

ALPHA-239= .2690 $(.10 .38)$

PU239 FOR:ED FROM UP3E=-.5078-03(.12-03)

INPUT DATA

CESIUH-137 DECAYS $=.2395+1(.2790+08) 0 / \mathrm{M} / \mathrm{Ml}$.

PU DECAYS $=.56 .5+08(.1017+7) \mathrm{D} / \mathrm{M} / \mathrm{ML}$

F'ERCEI T LECAYS Y PU238 $=1 ;, 90$

SAMPLE $\because T=19.236 \mathrm{M} \quad C L: D W T=2.761 \mathrm{G}^{\mathrm{M}}$

ASSUMPTIONS

SOLUTIO: VCL $=51.9 \mathrm{ML}$

WOL PU241 TIECYY CORRECTIO TIME = .309YEATS

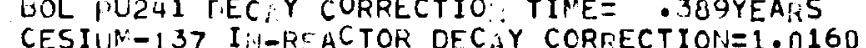

-

CESIUM- 137 HALF LIFE $=29.68(.10)$ YEARS

PU241 HALF LIFE $=14.5(0.5)$ YEARS

ALPHA241 $=.370(.030)$ 
ELEMEIT:135 ANAL:SIS OF THE SAMPLE 74.50 INCHES FWU: THE TOP OF THE MIDDLE ROD

$$
\text { I } 50 \text { T O P I C I NVEN TO : } Y
$$

\begin{tabular}{|c|c|c|c|}
\hline $\begin{array}{l}1 S U C F L \\
v-234 \\
v-235 \\
v-238 \\
v-236\end{array}$ & $\begin{array}{l}\text { fOL }(6 \cdot 1) \\
.4776-04 \\
.7076-02 \\
.0000 \\
.9581-00\end{array}$ & $\begin{array}{l}\text { E L MASS RA } 10 \\
.419-04(.23-05) \\
.5 \times 06-02(.357-04) \\
.3,502-03(.71-05) \\
1.0000\end{array}$ & $\begin{array}{r}\text { EOL }(A / O) \\
.0049(.0002) \\
5276(.0035)\end{array}$ \\
\hline
\end{tabular}

TUTAL $\quad .9052-110$ 
ELEMEIIT $\$ 185$... ANALYSIS OF THE SAMPLE 84.50 INCHES EROM THE TOP OF THE MIDDLE ROD I SOTOPIC I N V E N T O Y Y

\begin{tabular}{|c|c|c|c|c|}
\hline $\begin{array}{l}\text { ISOTCPL. } \\
U-C 34 \\
U-C 35 \\
U-236 \\
U=238 \\
\text { IOTAL }\end{array}$ & $\begin{array}{l}301 \quad(\mathrm{GM}) \\
.4976-04 \\
.7076-02 \\
.0000 \\
.9881-00 \\
.9952-00\end{array}$ & $\begin{array}{l}\text { EOL MASS PATIO } \\
.5141-04(.251-05) \\
.5008-02(.34-04) \\
.2282-03(.40-05) \\
1.0000\end{array}$ & $\begin{array}{r}\text { EOL }(A / 0) \\
.0051(.0002) \\
.5773(.0034) \\
.0227(.0004) \\
99.3949(.0035)\end{array}$ & $\begin{array}{c}\text { EOL (GM) } \\
.4987-04(.249-05) \\
.5659-02(.344-04) \\
.2233-03(.406-05) \\
.9914-00(.137-03) \\
.9974-00(.132-03)\end{array}$ \\
\hline
\end{tabular}

NET PRODUCTIOR: (GM) $.1125-06(.240-05)$ $-.1417-02(.344-04)$ $.2233-03(.406-05)$ $-.1368-02(.137-03)$
$-.2567-02(.132-03)$

150 TOFL
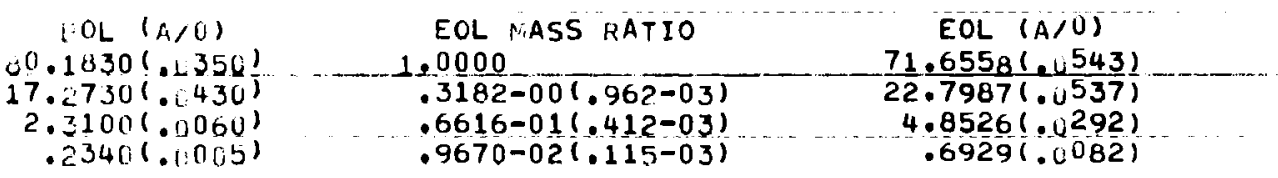

SPECIFIC ACTIVITY (D/M/GM) $.13616+12(.170+09)$ $.50130+12(.300+10)$ $.57600+10(.100+09)$ $.87000+1)(.200+09)$

E(L (GM)

$.3562-02(.8070-04)$ $.1138-02(.2577-04)$ $.2432-03(.5718-05)$ $.34 .7-04(.8929-06)$ $.4978-02(.1126-03)$

55.1
$5 / M T M)$
.8
.8
.9
.1

\section{BOL (GM)}

$3825-02(.1929-04)$ $.8275-03(.4041-05)$ $.1132-03(.6441-06)$ $.1130-04(.6165-07)$ $.4777-02(.2400-04)$

PU⿺⿻一𠃋十

TOTALS

$\begin{array}{ccc}\text { ISOICFL } & \text { FISSICIS (GN) } & \text { EURNUP }(M W N / M T M) \\ U-355 & .1190-02(.3462-04) & 1142.8 \\ U-23 E & .1515-03(.1511-04) & 148.5 \\ \text { HUSC } & .9583-03(.7122-04) & 944.8 \\ \text { PUC41 } & .6344-04(.5080-05) & 62.9 \\ \text { TOTALS } & .2364-02(.6475-04) & 2299.1\end{array}$

$U C 35 / U C 3.5 Y: J L=.7907(.01748) \quad$ ALPHA-2.55=.195.6(.006)

INPUT DAT

CESILA- 137 DECAYS $=.8040+D S(.1768+08) \mathrm{D} / \mathrm{M} / \mathrm{ML}$

PU DLCAYS $=.5 B, 0+U 8(.2176+, 7) \mathrm{D} / \mathrm{M} / \mathrm{ML}$

PERCETIT LECAYS LY PU23 $8=1 i_{i}, 40$

SAMPLE TT=16.106N CL D WT $=2.3856 \mathrm{M}$

SOLUTIOI: VOL $=3 \%$. $8 M L$

ALPHA-239= $.5505(.15,37)$

TIME OUT OF RIACTOR= .488YRS

BOL FU241 DEC,YY CORRECTIO TIME = .389YEARS

CESI HY-137 I

- - - VIAITITIES ENCLOSED BY PARENTHESES ARE ONE STANUARO DEVIATION ERRORS--.-- 
ELEME:T -1:5 AIIAL,SIS OF THE SNMPLE 2.50 INCHES FHO THE TOP OF TAE OUTSI,IE ROD

I $S$ O T C P I C I N V E N T O I Y Y

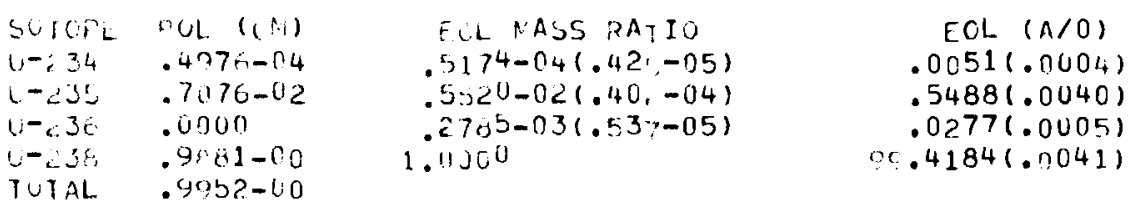

- $9081-00$

$.9052-60$

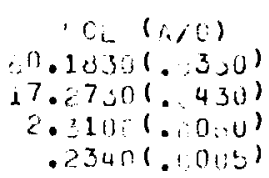

\section{F I S S I 0 is S}

\section{EOL $(A, 0)$ $68.6922(.735)$ \\ $2.4 .9215(\cdot .752)$ \\ $5.4500(.369)$ \\ .93631 .0102}

BOL $\left(G M_{1}\right)$

$3825-02(.1 \pm<9-04$.

$.8275-03(.4041-05)$

$.1132-03(.6441-06)$

$.1130-04(.0105-07)$

$.4777-02(.2400-04)$

DURNUP ( $\because W / M T M)$

1367.8
165.6
1616.1
99.6
3249.2

1367.8
165.6
1616.1
99.6
$3>49.2$

FISS, ON YIELL .062 (.0014) .062 u $(.0060)$ $.0674(.0019$ $.006 u(.0033)$
EOL $(\mathrm{GM})$ - $15-04(.422-05)$ . 373-n2(.402-04)

$722-n 3(.534-05)$

$905-(10(.156-03)$

$965-00(.151-03)$
IET PROOUICTIOH: (6⿴囗十) - 3375-06(.428-05) $-.1703-02(.402-04)$ $.2722-03(.534-05)$ $-.2259-02(.15 v-03)$

$-.3696-02(.151-03)$

$$
\begin{aligned}
& U-238 \\
& \text { HC3S } \\
& \text { FUC41 } \\
& \text { TUTALS }
\end{aligned}
$$

SPECIFIC ACTIVITY (L/N/GM) $.13016+121.170+09^{\circ}$ $.5 \mathrm{~J} 130+12(.300+10)$ $.57600+10(.100+09)$ $.87000+10(.200+0.0)$

MET PRODUCTION (GM)

$-.31) 22-03(.82 .60-(14)$

$.4500-031.2949-64)$

$.1680-03(.6743-05)$

$.3732-04(.1229-05)$

$.3598-03(.1199-03)$

LEL

$.0491(.0049)$

MEV/FISSIOI

$.0563(.0056)$

$.0604(.0060)$

PU239 FORNED FROM U>3 $3=.2099-021.15-031$

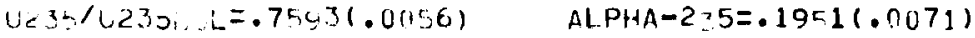

A1 PHA-239= .4651(.0977)

\section{ASSUMNTIONS}

CESI I I- 137 DE AYS = $1120+1.1 .2240+0810 / M / M L$

CESIUM- 37 HALF LIFE $=29.68(.1$ U) YEARS

PU IECAYS $=.5378+U 8(.1076+7) \mathrm{D} / \mathrm{M} / \mathrm{ML}$

PERCEINT DECAYS \& PU $238=11.40$

SANPLF WT=15.4756 $\mathrm{Mi} \quad$ CL D WT $=3.697 \mathrm{GM}$

PU241 HALF LIFE $=14.5(0.5)$ YEARS

ALPHAC $41=.370(.030)$

TINE OUT CF IFACIOR= .276、RS

SOL 11241 UEC YY LORFECTIO: TLME= 3ETYEARS

CESIUI-137 IT - REACTOK OECAY CORNECTIOH $=1.0160$

- 


\section{ELENETT 5105 ANALYSIS OF THE SAMPLE 12.50 INCHES FRON THE TOP OF THE OUTSIUE ROD}

ISOTOPIC INVENTORY

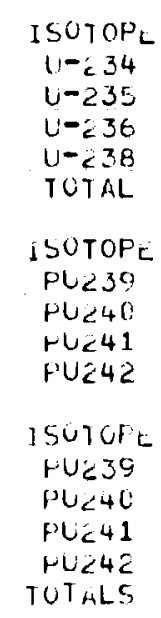
$.4976-04$
$.7076-02$ .0000 $.9881-00$ $.9281-00$
$.9052-00$

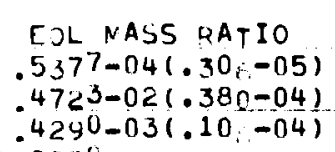

NET PRODUCTION (GN)
$.2293-05(.305-05)$

$-.2484-02(.376-04)$

$.4189-03(.107-04)$

$-.3423-02(.18 .7-03)$

$-.5504-02(.183-03)$

SOTOPE EOL $(A / O)$

$40.1830(.6354$

17.27301 .1430

2.31006 .6000

$.2340(.0005)$

$\begin{array}{cc}\text { EOL NASS RATIO } & \text { EOL }(A / 0) \\ 1.0000 & 64.0695(.0887) \\ .4048-00(.174-02) & 26.2592(.0857) \\ .1111+00(.112-02) & 7.3027(.0691) \\ .2418-01(.335-03) & 1.5685(.0215) \\ \text { ENL (GM) } & \text { BOL (GM) } \\ .357-02(.8199-04) & .3825-02(.1929-04) \\ .1462-02(.3322-04) & .8275-03(.4641-05) \\ .40(3-03(.1018-04) & .1132-03(.6441-06) \\ .8807-04(.2349-05) & .1130-04(.6165-07) \\ .5556-02(.1262-03) & .4777-02(.2400-04)\end{array}$

SPECIFIC PCTIVITY $(0 / \mathrm{M} / \mathrm{GM})$

$.13616+12(.170+09)$

$.50130+12(.300+10)$

$.57600+10(.100+09)$

$.87000+10(.200+09)$

NET PRODUCTION (GM)

$-.2280-03(.8423-04)$

$.6347-03(.3354-04)$

$.2951-03(.1020-04)$

$.7677-04(.2350-05)$

$.7786-03(.1284-03)$

FIS 5 I 0 NS

$1 S O T O P L$

U- 235

$1-<38$

H) 39

1.

FISSIGS (GN)
$.2051-02(.3911-04)$
$.2386-02(.2377-04)$
$.2214-02(.1328-03)$
$.2 J 00-03(.1791-04)$
$.47115-02(.1305-03)$

RURNIP (MWD/MTM)

1068.7

233.9

2182.7

204.9

4590.3

UK.S5/UC30, L $=.64,91.0053)$

ALPHIA-2:5=.2113(.0074)

ALPHA-239 $=.54 \Omega(.0772)$

FISSION YIELL

$.0628(.0014)$

$.0624(.0060)$

$.0674(.0019)$

$.066(.0033)$

DEL

$.0491(.0049)$

$.0563(.0056)$

$.0604(.0060)$

MEV/FISSIO
202
209
211
214

PU239 FORAED FROM U23R=.3197-02(.18-03) INPUT DAT,

CESIUPI-137 DECAYS $=.2358+1,(.471 E+08) 0 / \mathrm{N} / \mathrm{ML}$

PU DECAYS $=.92: 8+08(.1846+7) D / M / M L$

PERCLNT DECAYS IY PU238=1:10

SAMPLE IT TE2. $.656 \mathrm{M} \quad \mathrm{CL}, D W T=3.281 \mathrm{GM}$

SOLUT IOH VOL $=56.4 \mathrm{ML}$

LL, U WI $=3.2016 \mathrm{M}$

SOLUTIOH VOL $=56.4 \mathrm{ML}$

TIME OUT OF KI ACTOR= .276, RS

BOL PU241 DECAY CORRECTIO TI:E= .339YEARS

CESILM-137 I H-REACTOK DEC YY CORFECTIONI 1.0160

ASSUMPTIONS

CESIUM- 37 HALF LIFE $=29.68(.10)$ YEARS

PU241 HALF LIFE $=14.5(0.5)$ YEARS

ALPHA24I $=.370(.030)$

----OIAIATITIES ENCLOSED BY PAFENTHESES ARE ONE STANDARD DEVIATION ERRORS----- 
ELEMEA T 1.5 AIJAL, SIS OF THE SAMPLE 23.50 INCHES firu. THE TOP OF the OUTSILE ROD

$$
\text { I C O T O P I C I N V E N T O : Y }
$$

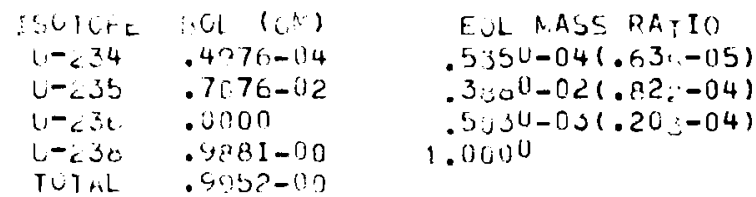

$E C L(1 / 0)$
$.0153(.0006)$
$.3063(.0082)$
$.0501(.0020)$
$.99 .5583(.0084)$
EOL $(\mathrm{Gl})$
- $173-04(.032-05)$
$.3767-(.02(.816-04)$
$.4905-03(.202-04)$
.$=981-00(.237-03)$
$.925-00(.222-03)$

$$
\begin{aligned}
& \text { EUL ASS :ATIO } \\
& 1.0000 \\
& .4526-00(.137-02) \\
& .1357-00(.998-03) \\
& .3665-01(.597-03)
\end{aligned}
$$

$E(\mathrm{~L}$ (GM)

$.3647-02(.8298-04)$

.16r 7-02(.3763-04)

.50.6-03(.1200-04)

$.53 .6-03(.1209-04)$

.59:5-02(.1350-03)
:SUICFE :OL $($, i, i,

rus $3: 4$

$+i_{c}$ is

1. $0<1$

$10<42$

ISUTCFE

Fuc 39

P. 40

$1 U_{c+1}$

$P U \leq 4<$

IUTALS

$$
\begin{aligned}
& 1.1236(.356) \\
& 17.27301 .436 \\
& \text { 2. } 10 \mathrm{cl} \cdot 00 \mathrm{u} \\
& \text { - } 3+1:(\cdot 0,1,5)
\end{aligned}
$$

EOL $(A, O)$

$61.4741(.681)$

$27.8219(. .639)$

8.45091 .0076

$2.2530(.353)$

BOL (GN)

$.0825-02(.1,29-04)$

. $8275-03(.4541-05)$

$.1132-03(.6441-06)$

$.1132-03(.6+41-06)$
$1130-04(.0105-07)$

$.1130-04(.0105-07)$
$.4777-02(.2600-04)$

F I S S:ON 5 1.UICPE FISEIOS (GN)

$u-35$

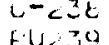

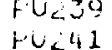

FUCL
TUIALS $.3108-0 ?(.3208-04)$ - $194-0$ (0) $\cdot 333,3-0 \leqslant(\cdot 2290-04)$
$.6316-02(.1741-03)$ BURNUP (NWF/MTM) 2676.1
313.7
2833.7
321.1
6154.5

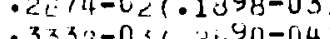

A. PHA-239= $.5846(.0689)$
IVET PROQUCTIOL (6.\%) $.1966-05(.63 z-05)$ $-.3309-02(.81 n-04)$ $.4905-03(.202-04)$ $-.4675-02(.237-0.3)$

$-.7526-1) 2(.262-03)$

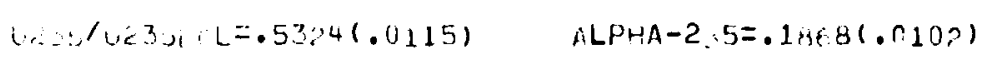
¿IPUT DAT:

CESIL -137 Dt CARS $=.3910+10(.7820+081 \mathrm{U} / \mathrm{N} / \mathrm{ML}$

(-1) $D=C_{A Y S}=.1 x_{i} Z+U 9(.2605+.7) D / M / M L$

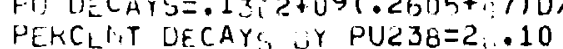

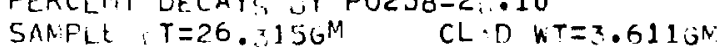

SOLUIIOI, VOL $=01.0 \mathrm{ML}$

TIME CUT OF :ACTOR= . 276:RS

BOL FU2'1 CECEY CORPECTIO: TIME= . X69YEAirS.

CESIU:-137 I,-W

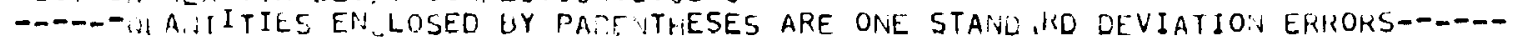

$\begin{array}{cc}\text { LEL } & \text { MEV/FISSION } \\ .0491(.0049) & 202 \\ .0563(.0056) & 209 \\ .0604(.0060) & 211 \\ & 214\end{array}$

$.13016+12(.170+(i)$,

$.50130+12(.3 n 0+10)$

$.57000+10(.1) 0+09)$

A.ET Pl:OUUCTION (SM)

-.17 \&2-03(.8519-04)

.8300-03(.3791-04)

$.3923-03(.1211-04)$

$.11<8-02(.1371-03)$ 
ELEMEN 5185 ANALYSIS OF THE SAMPLE 33.50 INCHES FROII THE TOP OF THE OUTSIDE ROD

$$
I S O T O P I C \text { I N V E N T O Y Y }
$$

\begin{tabular}{|c|c|c|c|c|}
\hline $\begin{array}{l}\text { ISUTOPL } \\
U-\angle 34 \\
U-235 \\
U-\angle 3 U \\
U-238 \\
T O T A L\end{array}$ & $\begin{array}{l}\text { HOL }(G M) \\
.4976-04 \\
.7076-02 \\
.0000 \\
.9881-00 \\
.9952-00\end{array}$ & $\begin{array}{l}\text { EQL MASS RATIO } \\
.4444-04(.230-05) \\
.3630-02(.311-04) \\
.5020-03(.12 ;-04) \\
1.0000\end{array}$ & $\begin{array}{r}E O L(A / 0) \\
.0044(.0002) \\
.3664(.0031) \\
.0560(.0013) \\
99.5732(.0033)\end{array}$ & $\begin{array}{c}\text { EOL }(G M) \\
.4296-04(.229-05) \\
.3572-02(.308-04) \\
.5479-03(.127-04) \\
.9878-00(.232-03) \\
.9920-00(.229-03)\end{array}$ \\
\hline
\end{tabular}

NET PRODUCTION (GM) -.6806-05 (.22s-05) $-.3504-02(.308-04)$ $.5479-03(.127-04)$ $-.5479-03(.127-04)$ $-.7950-02(.229-03)$

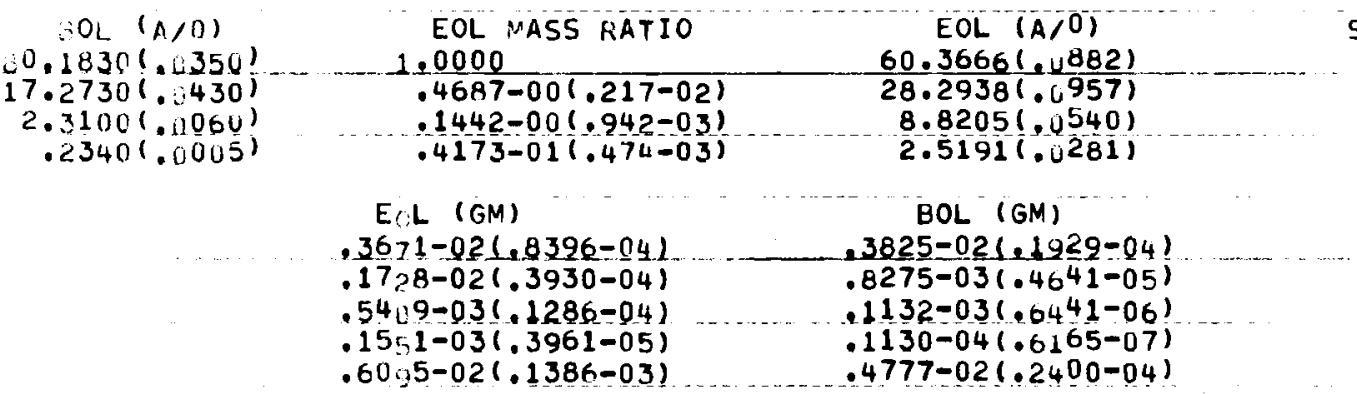

SCICFL

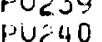

PU⿻

Puट4 1

ISOTOPL

Nu 39

$\mathrm{PLC4C}$

Pि241

$P \cup<42$

TOTMLS

$6005-02(.1386-03)$ 4777-02 (.2400-04)

SPECIFIC ACTIVITY (D/M/GM)

$.13616+12(.170+(09)$

$.50130+12(.300+10)$

$.57600+10(.100+09)$

$.87000+10(.200+09)$

\section{NET PRODUCT ION (GM)}

-.1541-03(.8614-04)

$.9004-03(.3957-04)$

$.4277-03(.1288-04)$

$.1438-03(.3961-05)$

$.1318-02(.1407-03)$

F I SS, ONS

ISUTUPL

$v-\div 35$

$\mathrm{v}-238$

PUट36

एण 24

TUTAL:
FISEI (:.5 (GN)

$.2921-0,1.3332-04)$

. 2341-0r (.1006-03)

.3271-03(.3315-04

$.6582-02(.1811-03)$
$.3334-03(.3313-04)$
FURNISP (MWO/MTM)

2803.8

327.0

2899.9
383.8

6414.4
ViCSOUGJUACL $=.50461 .0043$ ) ALPHA-2.5=.1906(.0056)

NPUT DAT

INPUT DATA
CESILM-137 DECAYS $=.3770+1 . .1 .7540+08) 0 / M / M L$

PU DE CAYS $=.1255+09(.2509+; 7) D / M_{1} / M L$

PEKCEIVT DECAYS ?Y PU238=2,.00

SAMPLE T=24.100GM CL:D WT $=3.254 \mathrm{GM}$

FISS ON YIELD
$.0628(.0014)$

$.0624(.0060)$

$.0674(.0019)$

DEL

$.0491(.0049)$

MEV/FISSIOR

$.0604(.0060)$

209

211

ALPHA-239=.63EL $(.0691)$

PU239 FORIVED FROM Uว $38=.4643-02(.23-031$

\section{ASSUMPTIONS \\ CESIUM- 137 HALF LIFE $=29.68(.10)$ YEARS \\ PU241 HILF LIFE $=14.5(0.5)$ YEARS \\ ALPHA241 $=.370(.030)$}

TIME OUT OF REACTOR= .276 TRS

BOL HU241 TECAY CORRECTIO:: TIME= .3B9YEARS

CESIUM-137 INI-REACTOR DECAY CORRECTION=1.0160

---- (CUANTITIES ENCLOSED BY PARENTHESES ARE ONE STANUARD DEVIATION ERRORS----- 


\section{I $S O O T O P$ I C INVEN T O}

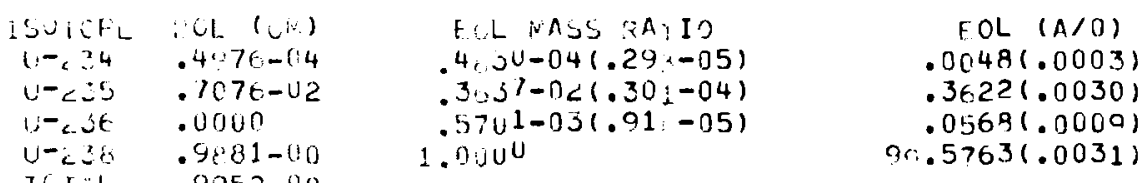

Toral $9831-00$ . 5 iv1-03(.91:-05) r. $5763(.0031)$

$$
\begin{aligned}
& \text { EOL. }(\text { Giv }) \\
& .4649-014(.292-05) \\
& .0515-02(.299-04) \\
& .0534-03(.914-05) \\
& .0635-00(.304-03) \\
& .0877-00(.302-03)
\end{aligned}
$$

EOL $(A / 0)$

\section{$60.3470(.8882)$}

$28.2846(. ., 957)$

$8.8501(.642)$

$2.5183(.281)$

BOL (GM)

$3825-0<(.1,329-04)$

$.8275-03(.4,41-05)$

$.1132-031.0441-06$

$.1130-04(.0+05-07)$

$.4777-02(.2400-04)$
1. $14<4$

TOT TLS

$.54 .2-02(.1254-1) ?$

. 2.5 . $0-02(.5869-0 i t)$

$.23,6-03(.5914-04)$

$.91 \cdot 4-02(.2071-02)$

F I S S T O in $S$

ISUlol tissIas (GM)

$1-<j$

$j-<3 B$

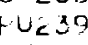

मU. 41

IUTALS

$.2+71-02(.3124-04)$

$.4,3 x-03(.4011-04)$

. $3524-021.2189-03)$

$.5530-03(.5065-04)$

$.7,91-02(.<189-43)$
FISS ON YIELD

$.0620(.0014)$

$.062 j(.0060)$

$.0674(.0019)$

.060 a (.0033)

$\begin{array}{rl}2851.6 & .0620(.0014) \\ 395.5 & .062)(.0060) \\ 3868.7 & .0674(.0019) \\ 588.1 & .0604(.0033) \\ 7703.8 & \end{array}$

EEL

$.0491(.0049)$

$.0563(.0056)$

$.0604(.13060)$
HET PROOUCTIU⿴囗十⺝ (5)

$-.3276-05(.29<-03)$

$-.3561-02(.2901-04)$

$.5534-03(.914-05)$

$-.0206-02(.304-03)$

$-.1225-01(.30 \div-03)$

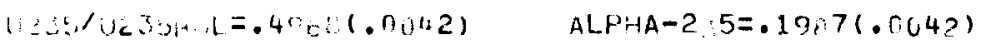

ALPHA-239= .4517(.07n,7)

PU239 FORAEU FROM U>33 $=.3839-02(.30-031$

\section{ASSUMPTIONS}

CESII $N-13$, ATM M $=.4733+1:(.9466+15)$ ATOMS $/ M L$

CESIUM- 37 HALF LIFE $=29.68(.1 \cup)$ YEAKS

PU241 iH:LF LIFE $=14.5(0.5)$ YEARS

ALPHAC $41=.370(.030)$

FERCLIT UECAY: 31 PU238=?;0 10

SAMFLE T=22.116M CL, D WT 1 M.160GM

SOLUTIOI: VOL $=26.5 \mathrm{ML}$

TINE OUT OF RIACTOR= .353 RS

UOL, U241 LECAY CURRECTIO, TIME = .389YEARS

CESIU -137 I - - LACTOR DEC, Y CORPECTION=I.0160

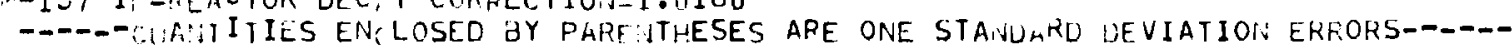


ELEMETT UIUS ANALYSIS OF THE SAMPLE 38.00 INCHES FRO THE TOP OF THE OUTSIDE POD

$I \subseteq O T O P I C$ I N VENTOHY

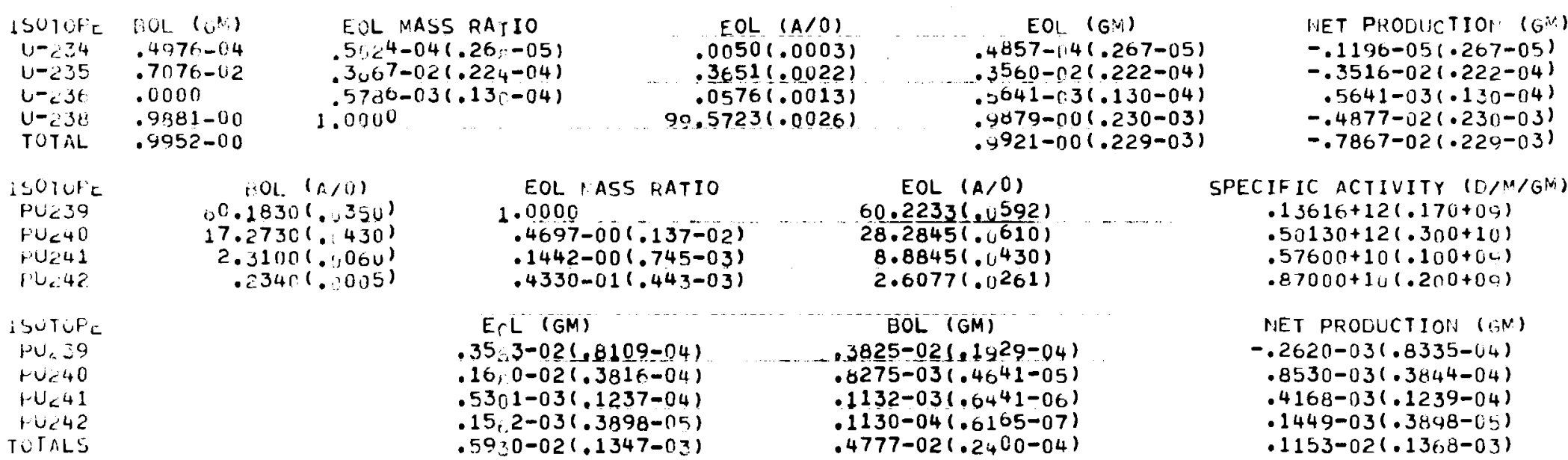

$F \perp S S$ I 0 N 5

\begin{tabular}{|c|c|}
\hline 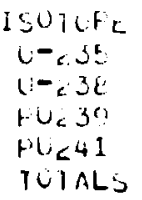 & 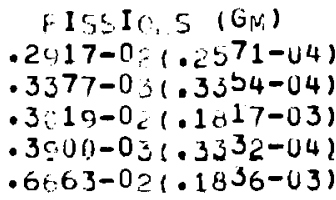 \\
\hline
\end{tabular}

E.URNIIP $\left(\cdot W_{C} / M T\right.$
2799.8
331.2
2976.0
386.8
6493.7

FISSION YIELO
$.0620(.0014)$
$.0624(.0060)$
$.0674(.0019)$
$.0664(.0033)$

$\begin{array}{cc}\text { CEL } & \text { MEV/FISSIOI: } \\ .0491(.0049) & 202 \\ .0563(.0(56) & 209 \\ .0604(.0060) & 211 \\ & 214\end{array}$

UC3b/L23D:CL=.5031(.06.21) ALPHAA-2.5 $=.2066(.0056)$

NLPHA-239= $.5979(.0651)$

PU239 FORMED FROM U23y $=.4558-02(.22-03)$

INPUT DAT:

CESIUN-i37 CRLAYS $=3823+1,(.764 E+0810 / N / M L$

PU DLCAYS $=.1_{C} \times 1+09(.2463+7) \mathrm{D} / \mathrm{M} / \mathrm{ML}$

PERCLP.T DECAYS ISY FU238=21.30

SAMFLE $W T=24.705 G \mathrm{M} \quad C L, D$ WT $=3.395 \mathrm{GM}$

ASSUMPTIONS

SOLUTIO VOL $=63.7 \mathrm{ML}$

BOL PU241 CECAY COKRECTIO: TIME = .389YEARS

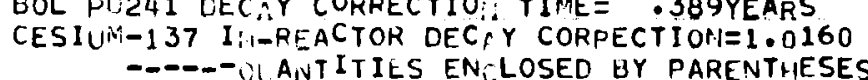

\section{CESIUM- 37 HALF LIFE $=29.68(.10)$ YEAKS}

PU241 HALF LIFE $=14.5(0.5)$ YEARS

ALPHA241 $=.370(.030)$

---- O ANTITIES EN LOSED BY PARENTHESES ARE ONE STANIJARD DEVIATION ERRORS------ 


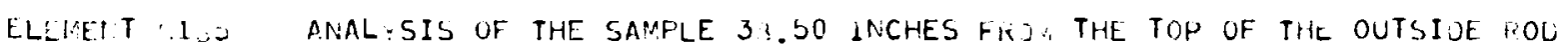

I $S O T O P$ I C I NVENTOHY

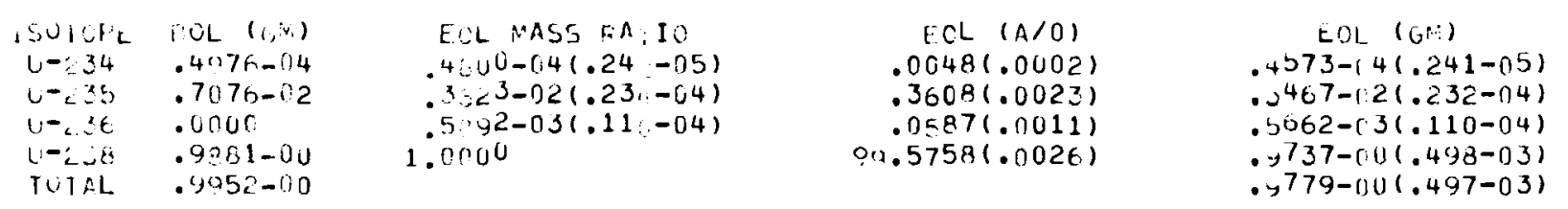

$.4052-0$
(ALT PFOCHCTIO (G) $-.4027-015(.241-05)$ $-.3609-1) 2(.232-04)$
$.5662-03(.110-04)$
$-.1905-(11(.49 s-03)$
$-.2213-01(.497-03)$

$$
\begin{aligned}
& O_{L}(\therefore, \pi) \\
& \text { se. } 123(-320) \\
& 17.2730(.430) \\
& \text { 2. } 310(0.1000)
\end{aligned}
$$

EOL :ASS :ATIO

$$
1.0000
$$

$.4715-00(.120-02)$

$1430-00(.71 ;-03)$

$.0306-01(.441-03)$

$\operatorname{EOL}(A, U)$

$60.1903(.562)$

$28.3850(., 575)$

$8.8267(.416)$

$2.5921(\ldots 260)$

E, L (GM)

$.94 .5-02(.1912-0.3)$

$.39(0-02(.9037-04)$

$.1243-0 ?(.2897-04)$

.36-5-03(.9141-15)

EOL (OM)

$\mathrm{r}^{2}<39$

fu< 40

Fit 4 2

TOT MLS

ron

ISUIOFL

(i- उb

L- $\angle 3 E$

PUL34

Huist

TOTALS

$.13 .9-01(.3178-0)$

F I S 5 I 0 iv 5

. $3825-02(.1$ y29-04) $.8275-03(.4 n, 41-05)$

$.1132-03(.0+41-06)$

$.1130-04(.5165-07)$

$.4777-02(.24 \cup 0-04)$

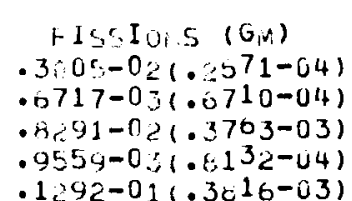

RURNUP ("IW: /MTA1)

29.4 .6

558.7

3174.6
047.9

12655.8

$1.35 / U 23$ U. L $=.4399(.0033) \quad$ ALPHA $-2 \times 5=.2011(.0047)$

ALPHA-239 $=.6740,(.0552)$

FISSION.YIELO
$.0620(.0014)$
$.0621(.0060)$
$.0674(.0019)$
$.0060(.0033)$

SPECIFIC ACTIVITY (U/N:GAH)

- $13016+12(.170+09)$

$.50130+12(.300+10)$

$.576(j 0+10)(.100+04)$

$.87000+10(.200+0.1)$

TET PRODUCTION (C.M)

$.4530-02(.1922-0,3)$

$.3152-02(.9049-(14)$

$.1130-02(.2897-014)$

$.3552-03(.9141-05)$

$.9217-02(.31 \times 7-0.3)$

CFSIL, 137 ATR $=.8343+1 \% 1.1669+161 A T O M S / M L$

PU [LCAYS $=.14 .4+\cup 9(.2968+7) D / M / M L$

FEFCEIT OHCAY: Y PUL38 $=23.50$

SAMPLE : $T=23.2116 \mathrm{M} \quad C L: O W T=3.1456$

ASSLIMPTIONS

CESIUM- 137 HR.LF LIFE $=29.6001 .10)$ YFARS

PU241 HULF LIFE $=14.5(0.5)$ YEAPS

ALPHA241 $=.370(.030)$

SOLUTIO VOL=

TINE OUT OF DOCTOR=.529 RS

WOL UU2'1 LEC.Y CORRECTIOH TIME= .399YCAES

CFSIUN-i 37 I II-REACTOR DECAY COREECTION=1.0160

-i37 IIIFACTOR DECAY CORHECTION=1.0160 
ELEMENT E135 ANALYSIS OF IHE SAMPLE 44.75 INCHES FROH THE TOP OF THE OUTSIDE ROD

I SOTOPIC INVENTOKY

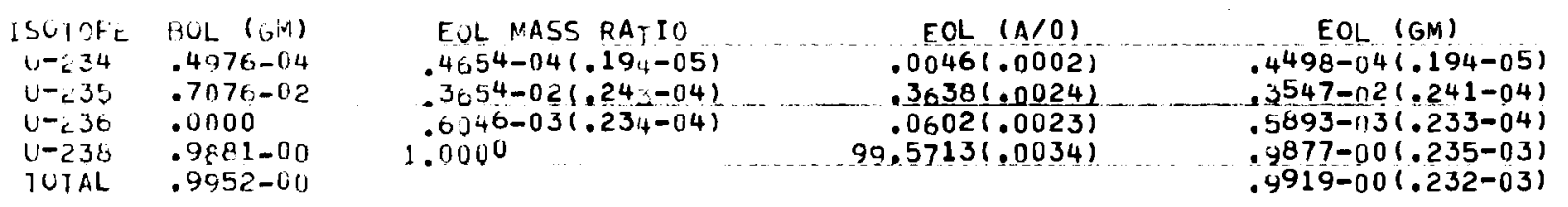

INET PRODUCTION (GM) $-.4782-05(\cdot 194-05)$ $-.3529-02(.241-04)$

$.5893-03(.233-04)$

$-.5084-02(.235-03)$

$-.5084-02(.235-03)$
$-.8063-02(.232-03)$

ISUTOPE POL $(\dot{A} / 0)$

PUE39 $\quad 0.1830(.1350)$

Puc40 $17.2730(.4430)$

Pu<41 $2.3100(.000)$

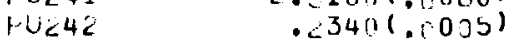

$\begin{array}{lc}\text { EOL NASS RATIO } & \text { EOL }(A / 0) \\ 1.0000 & 60.2067(.0562) \\ .4715-00(.129-02) & 28.3899(.0575) \\ .1430-00(.715-03) & 8.8109(.0414) \\ .4306-01(.441-03) & 2.5925(.0260)\end{array}$

E. L (GM)

$.3634-02(.8267-04)$

$.17<1-02(.3907-04)$

$.53 \in 3-03(.1250-04)$

. 15.4-03(.3952-05)

$.6049-02(.1374-03)$

FIS S I ONS

$\mathrm{BOL}$ (GM)

$.3825-02(.1 .929-04)$

$.8275-03(.4641-05)$

$.1132-03(.6441-06)$

$.1130-04(.6165-07)$

$.4777-02(.2400=04)$

FISSI (I:S (GM)

$.2904-02(.3353-04)$

$.3419-03(.3398-04)$

$.3094-02(.1853-03)$

$.3560-03(.3383-04)$

$.6740-02(.1800-03)$
FURNUP (MWO/MT:1)

2788.1

335.3

3054.0

392.7
6570.1

6570.1
FISSION YIELD

.062 \& $(.0014)$

$.0024(.0060)$

$.0674(.0019)$

$U_{C} 13 / U 23$ ji.CL $=.5012(.00 \geqslant 4)$

$A L P 1: A-235=.2152(.0090)$

ALPHA-239 $=.6003(.0647)$

SPECIFIC ACTIVITY (D/M/GM)

$.13616+12(.170+00)$

$.50130+12(.300+10)$

$.57600+10(.100+09)$

$.87000+10(.200+04)$

NET PRODUCTION (GM)

$-.1912-03(.8459-04)$

$.8932-03(.3934-04)$

$.4230-03(.1251-04)$

$.1471-03(.3952-05)$

$.1272-02(.1395-03)$

INPUT DATA

CESI V V- 37 DECAYS $=.3393+1.1 .678 E+08) \mathrm{L} / \mathrm{M} / \mathrm{ML}$

PU DECAYS $=.11(2+U 9(.2204+7) \mathrm{D} / \mathrm{M} / \mathrm{ML}$

PERCENT DECAYS BY PUI238=2:10

SAMPLE ${ }_{1} T=22.794 \mathrm{GM} \quad \mathrm{CL}: \mathrm{D} W \mathrm{TT}=3.744 \mathrm{GM}$

UEL

$.0491(.0049)$

MEVIFISSION

$.0563(.0056)$

202

$.0604(.0060)$

209

211
214

PUC39 FORMEO FROM UP38 $=.4763-02(.23-03$ )

ASSUMPTIONS

CESIUM-137 HALF LIFE $=29.681 .10)$ YEARS

PU241 HALF LIFE $=14.5(0.5)$ YEARS

ALPHA241 $=.370(.030)$

SOLUTIOA, VOL $=263.7 \mathrm{ML}$

TIME OUT OF HEACTOR= $488 Y R S$

BOL HU241 DECAY CORRECTIO TIME = 389 YEARS

CESIUNi-137 I I-REACTOR DEC.Y CORRECTION=1.0160

--- GHAITITIES ENCLOSED BY PARENTHESES ARE ONE STANOARD DEVIATION ERRORS---- 
FLLMETJT, 1.J MIVLLSIS OF THE SAMPLE 45.25 IIICHIES FRO. THE TOP UF THE OUTSIIJE FUD

$$
\text { I S O T, D I C I N V ENTO, Y }
$$

\begin{tabular}{|c|c|c|}
\hline$\therefore$ & & \\
\hline $11-34$ & 4075 & IL AASS TPAI10 \\
\hline$u=35$ & $.7076-02$ & $41=-04$ \\
\hline$u=c t$ & - unor & $12-041$ \\
\hline $11-<38$ & $.9,331-10$ & $1.000^{0}$ \\
\hline IUI I.L. & . प्युse & \\
\hline
\end{tabular}

COL $(A / 0)$
$.0056(.0004)$
$.3602(.0041)$
$.0586(.0012)$
$.5556(.0043)$

EOL $\left(G v_{1}\right)$ $.0356-(4(.354-n 5)$ $.0443-c 2(.414-04)$ $. \therefore 629-13(.123-04)$ $. j 688-00(.594-03)$ $. .729-(.01 .592-03)$

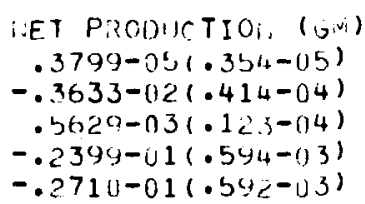

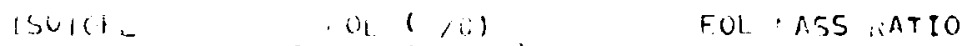

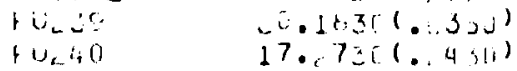

$+0.410 .10(1.206)$

$\cdot .3+47(\ldots, 0)$

$$
\begin{aligned}
& \text { FUL } 1.55 \text { :ATIO } \\
& \begin{array}{l}
.0000 \\
.4697-00(.137-0 \%) \\
.1442-00(.745-03) \\
.4330-01(.447-03)
\end{array}
\end{aligned}
$$

C. L $(G M)$

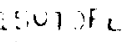

Hut

P.

inc $u$

1. 14 ic

(a) is

$.10: 2-01(.2302-0 ?)$

$.47-1-02(.108 ?-0 ?)$

$.150 .5-02(.3511-04)$

$.446-0.3(.1107-04)$

.16 4-01(.3(24-?)

$$
\text { C I S S } 101.5
$$

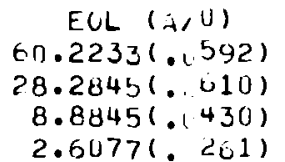

$$
\triangle C L(G M)
$$

$.3825-02\left(.1<<<9-04_{4}\right)$

$8275-03(.4,41-05)$

$.1132-03(.0441-06)$

$.1130-04(.0105-07)$

$4777-02(.2400-04)$

isule

$-\cos$

$1-58$

1. ve 39

vicit 1

$I \Leftrightarrow, I_{1} \leqslant(0,$.

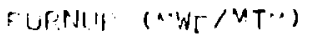

शก)

771.0

3047.7

1153.7

14791.9
F15S, ON YIEL:

$.0022(.0014)$

$.0624(.0050)$

$.007+(.0019)$

.06ol $(.0033)$

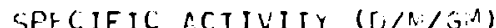

$.13016+12(.170+0 u)$

$.50130+12(.300+10)$

$.57000+16(.1110+(19)$

$.87000+10(.21,0+0$

NET PSODUCTIDH $(, \cdots)$

$.6292-02(.2310-(1.5)$

$.39344-0.21 .1(1,34-(1.5)$

$.1352-02(.3514-(1.4)$

$.4323-03(.1107-011)$

$.1206-01(.3431-0.3)$
[E,

$.0491(.15049)$

$.0563(.1305 n)$

$.0604(.0050)$
ME "/FISSIR:

202

204

214

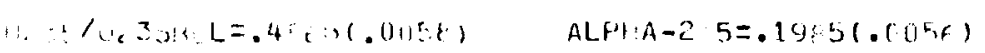

$\therefore$ i. PHA-239=.601:(.01547)

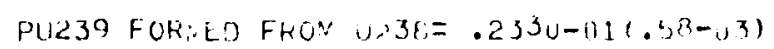

$$
\text { ASSIIHPT IUIV; }
$$

CESIUIN- 37 HILF LIFE $=29.601 .10)$ YEAFS

PU241 H, LLF LIFE $=14.3(0.5)$ YLAFS

ALPRAA241 $=.370(.030)$

PI: i C CAYS $=.9+3+C(.1336+7) \mathrm{U} / \mathrm{M} / \mathrm{ML}$

PERCL TECAY Y PLICZSE . 20

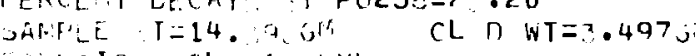

OLLIID+ VCL $=1$.UNL

TIP OUT 1,F A A IOR $=.477$ RS

,CL, 1'241 EEC.Y CURRCCTIO. TI"ME= .389YEA, 5

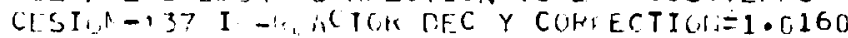

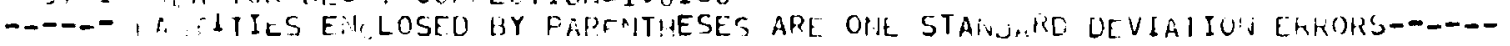


ELEMENT S186 ANALYSIS OF IHE SAMPLE 54.00 INCHES FROV THE TOP OF THE_OUTSIDE ROD

I SOTOPIC INVENTOHY

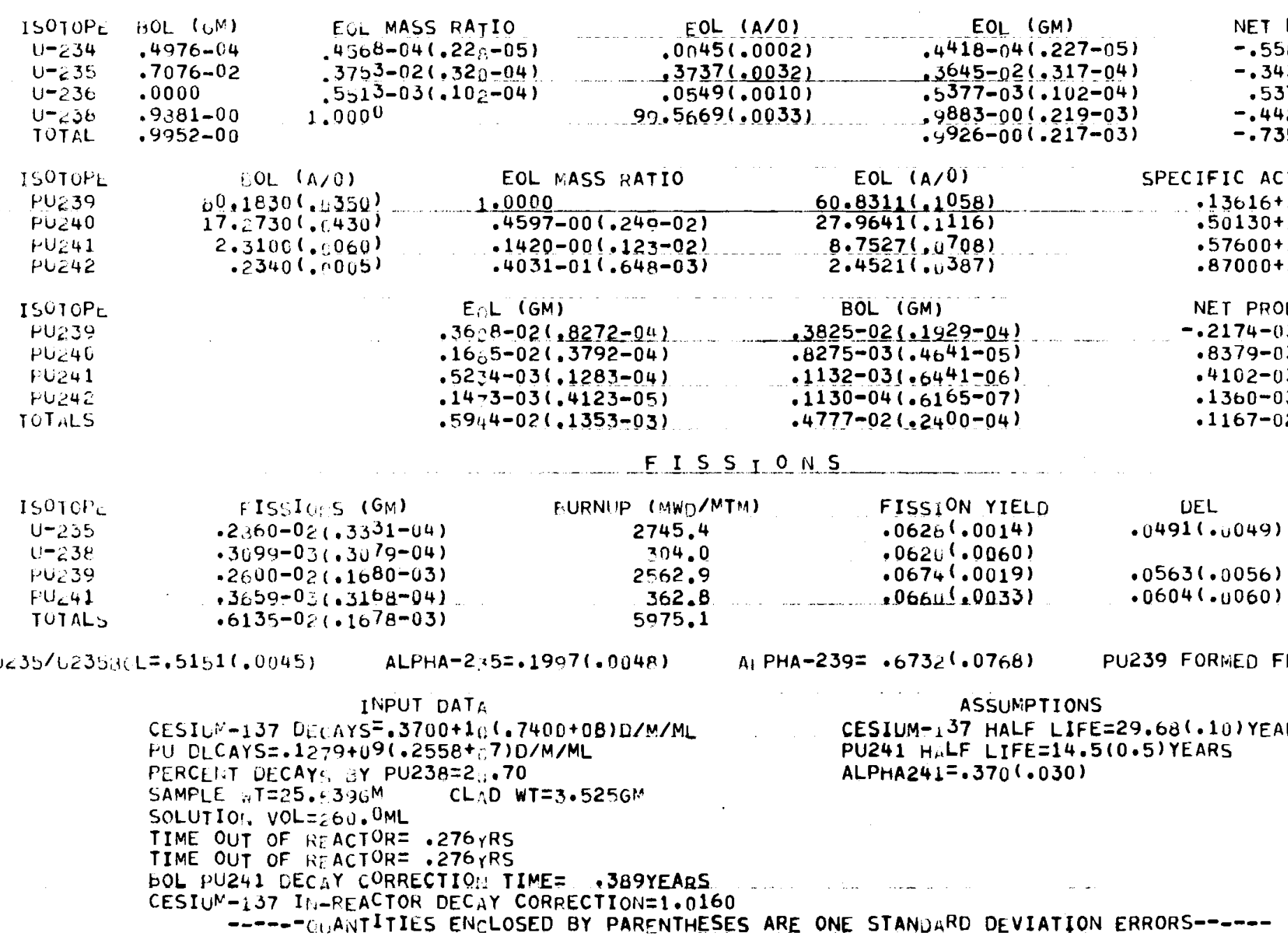




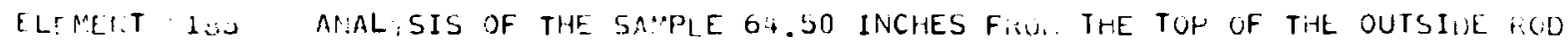

$$
I \subseteq O T O P I C \text { I NVE N T O }
$$

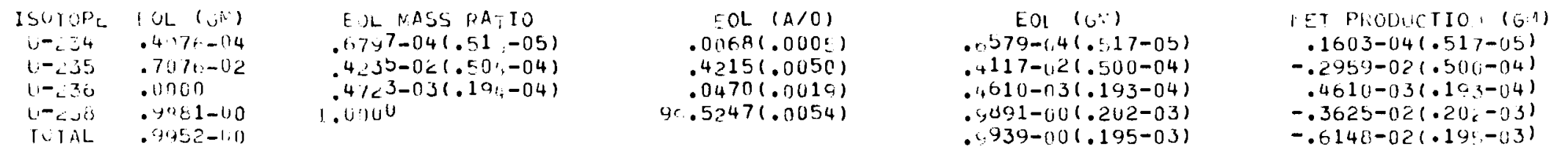

:(4. $(i ; 0)$

$0.16 .51(.230 u)$

$17.27 .30(.430)$

- $110(1,0,0,0)$

- 341, . (1900)

P. 42

Isulute

PU⿺廴⿻肀二𠃋

ive 40

Fi: $U_{c-4}+1$

$10,4 a$

U1iLS

LSUIOFE

$1.3-35$

$0-\therefore 8$

FU⿺廴十

IOTAL:
EOL NASS INATIO

1.0000
$.4267-00(.145-02)$

$.4267-00(.145-02)$
$.1270-00(.690-03)$

$.3143-01(.334-03)$

F. L ( M)

$.34,7-02(.7057-0 ! 3)$

$.148-02(.34 n 1-044)$

$.4536-0.3(.1062-1) 6)$

. $11,3-0 \geq(.2704-n k)$

.55: 0-0? $(.1262-0)$
EOL $(A / 0)$

$63.0193(.0655)$

$26.8928(. ., 681)$

$8.1072(.418)$

$1.9807(.0207)$

BOL (UN)

$.3825-02(.1929-1) 4)$

$.8275-03(.4041-05)$

$.1132-03(.04+1-06)$

$.1132-03(.04+1-06)$

$.1130-04(.5105-07)$
$.4777-02(.2400-04)$

F I S S: ONS
HURNLP $($ "W: $/ N T+n)$

$$
\begin{array}{r}
2376.7 \\
264.2 \\
2287.5 \\
266.8 \\
5105.3
\end{array}
$$

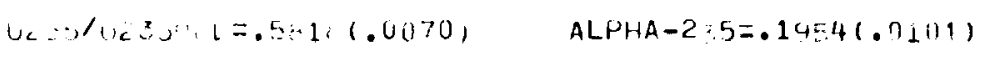

$L_{n} P H A-239=.594 .9(.0771)$

SPECIF IC ACIIVITY $(\mathrm{L} / \mathrm{V} / \mathrm{G}$ in)

$.13 t 16+12(.170+1)(\%)$

$.50130+12(.3: 00+13)$

$.57600+1(1.1(1)+11 ; i)$

$.87000+10(.200+00)$

I:F I PHODLCTION (an)

$-.3260-031.8187-0.3)$

$.6709-03(.3433-1,34)$

$.3404-03(.1064-04)$

$.9997-(141.2745-1) t)$

\begin{tabular}{|c|c|c|}
\hline $\begin{array}{l}\text { FISS ON YIELU } \\
.062 \mathrm{C}(.0014)\end{array}$ & $\begin{array}{c}\text { LEL } \\
.0491(.0049)\end{array}$ & $\begin{array}{c}\text { inE } \because / F I 3 S I O H \\
212\end{array}$ \\
\hline $.0620(.0060)$ & & $2 i 14$ \\
\hline $\begin{array}{l}.0674(.0019) \\
.0004(.0033)\end{array}$ & $\begin{array}{l}.0563(.0056) \\
.0604(.0060)\end{array}$ & $\begin{array}{l}211 \\
214\end{array}$ \\
\hline
\end{tabular}

$73<7-1) 3(\cdot 1<\cdot 14-1) 3)$

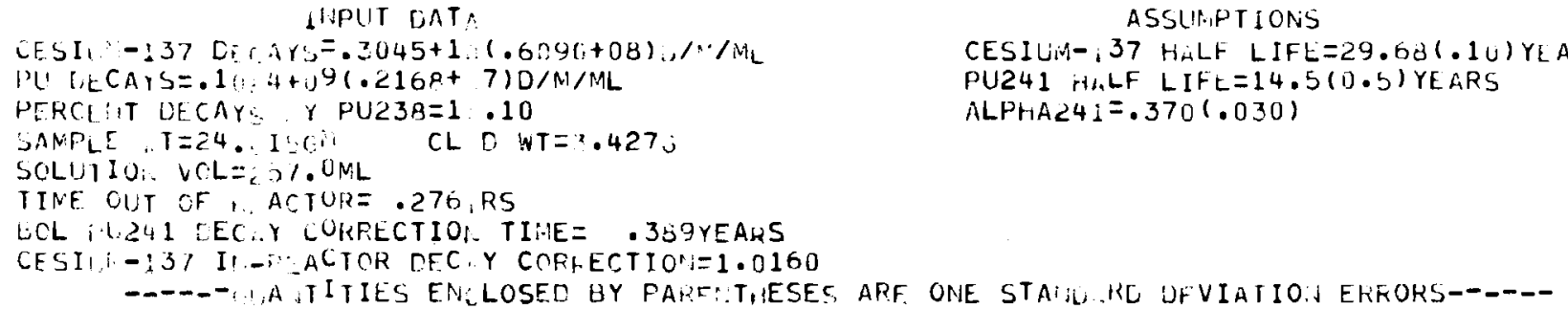

SOLUTIO, VCLZZOOOML

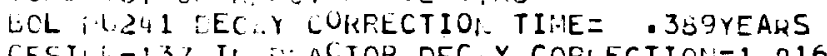

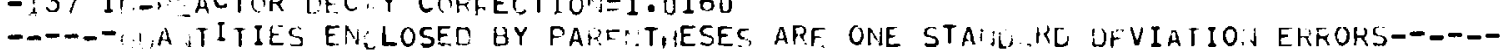

PU230 FOR:EL FFOM U. $38=.337(-02(.20-03)$ 
ELEMENT L 105 ANALYSIS OF THE SAMPLE 65.00 INCHES FROI IHE TOP OF THE OUTSIDE ROD

ISOTOFIC INVENTOKY

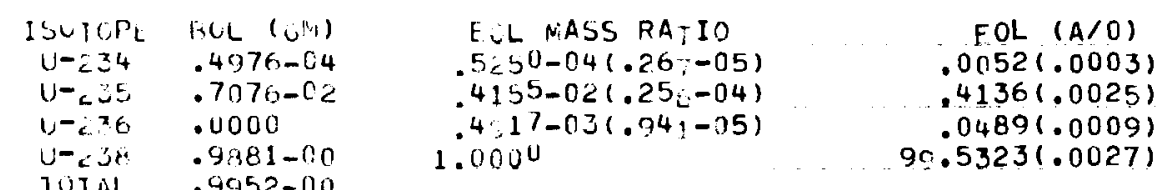

TUTAL $\quad .9981-00$ OOL $(n / 0)$
$10.1530(.350)$
$17.2730(.450)$
$2.3100(.0060)$
$.340(.0005)$

\section{EOL MASS RATIO} 1. 0000

4267-00(.145-02) $.1270-00(.699-03)$ $.3143-01(.3311-03)$

\section{E: $L(6 M)$}

$.62288-02(.1419-03)$ $.2673-02(.6068-04)$ $.811-03(.1902-04)$ .81 -03(.1902-04) $.195-03(.4985-05)$
$.9921-02(.2252-03)$

$$
\begin{gathered}
\text { EOL (GM) } \\
.5049-04(.266-05) \\
.4013-02(.254-04) \\
.4769-03(.936-05) \\
.9827-00(.310-03) \\
.9874-00(.309-03)
\end{gathered}
$$




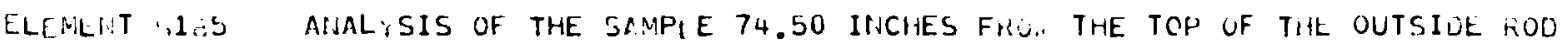

I $C$ O T O P I C INVE NTO

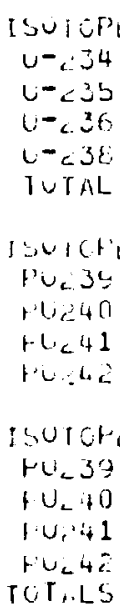

TCr..LS $u-c 5$

$u-28$

$+0.2=9$

IUTCL
EOL $(A / O)$ $.0047(.0003)$ $.0900(.0053)$ $.0 .384(.0009)$

$00.4669(.0054)$

.0000 $4.10-04(.27$ T $.49<6-02(.53-04)$

$310^{4}-03(.94,-05)$

$.91861-00$

1.0100

EOL $(6,1)$ $.4529-04(.277-05)$ $.4791-02(.532-04)$ . 774-r3(.041-03) . $8897-30(.180-03)$ $.951-00(.172-03)$

$$
\begin{aligned}
& \text { IIFT PROBIATICi, (Giv) } \\
& -.4474-05(.277-05) \\
& -.2285-02(.53,-04) \\
& .3774-03(.941-05) \\
& -.3011-02(.14(-05) \\
& -.4932-02(.17,-03)
\end{aligned}
$$

$$
\begin{aligned}
& \text { OL }(\therefore, 0) \\
& \text {. } 0.1+3 ! 1(.1330)
\end{aligned}
$$

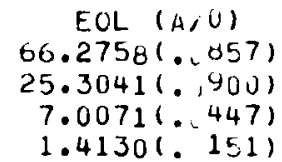

$\mathrm{BOL}$ (GM)

. उ825-0ट (.1., $<9-04)$

$.8275-03(.4,41-05)$

$.1132-03(.0+41-0)$

$.1130-04(. .105-1) 7$

$.4777-02(.2400-04)$

F I S S I ONS

I IS: I 5 (GM) $.1 .06-0 ?(.0403-04)$

- $2120-0 .(.2124-04)$

- $1005-0 \%\left(.12^{48} 8-03\right)$

$.1 .111-0 ?(.1503-04)$
$.4194-0 ;(.1157-03)$

HURNIP $(\cdots W-1 M T \cdot \cdots)$

1019.6
297.9
1978.2
179.6
4085.3

4471

SPECIFIC ACTIVITY ([ / A/Gin)

$.13015+12(.170+(14)$ $.5: 113 n+12(.3 n 0+16)$ $.57000+10(.100+64)$ $.87000+16(.200+0 i)$

RET PFOULCTIOH (i, $(\%)$ - .1942-031..5024-(t)

$.5685-031.32160-(14)$ $.2750-03(.928,1-05)$ $.6730-1) 4(.1943-05)$

$.7265-(31.1272-(.3)$

FISSIUN.YIELL
$.0020(.0014)$
$.0620(.0060)$
$.0674(.0019)$
$.0060(.0033)$

LEL

$.0491(.0049)$

$.0563(.0056)$

$.0604(.0060)$

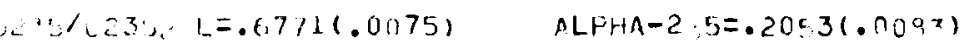

1) PHA $-239=.573 \times(.0391)$

PUL3O FOPAE? FROH (1238 $=.2811-02(.13-03)$ LINUT DAT:

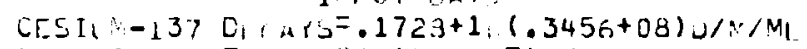
Pi) T.ECAYS $=.7318+U B(.1464+7) D / N / M L$ HEKCF OT UECAT O PU23H=11.080 SAMPIE T=17. $7 \mathrm{~K} G \mathrm{GM} \quad \mathrm{CL} D W T=2.474 \mathrm{U}$ ASSIJMTTIONS

CESIUN- 37 HALF LIFE $=29.60(.10)$ YE ARS PU241 HALF LIFE $=14.5(0.5)$ YEARS ALPHAC41 $=.370($. OSO $)$

$5 O L I 17$ IOL VOL $=05.5 \mathrm{ML}$

TIME UUT OF :ACTOR= .276;RS

BOL H241 LEL CY CORRECTIO, TINE= . ILYYEAFS

CESIL:-137 I: -RFACTOR DEC: Y CORPECTIOUI=1. 160

-... 
ELEMENT 5195 ANAL SIS OF THE SAMPLE 84.50 INCHES FROH THE TOP OF THE OUTSIDE ROD

I 5 O T O P I C I N VEN TO F Y

\begin{tabular}{|c|c|c|c|}
\hline $\begin{array}{l}\text { ISUTOFE } \\
U-\angle 34 \\
U-\angle 35 \\
U-36 \\
U-x S\end{array}$ & $\begin{array}{l}.0 L(G \mathrm{~V}) \\
.4076-04 \\
.7076-02 \\
.0000 \\
.9881-00\end{array}$ & $\begin{array}{l}\text { EOL MASS DATIO } \\
.5317-04(.32-05) \\
.6745-02(.511-04) \\
.2055-03(.61-05) \\
1.0000\end{array}$ & $\begin{array}{c}E O L(A / 0) \\
.0058(.0003) \\
.6698(.0050) \\
.0208(.0006) \\
90.3036(.0051)\end{array}$ \\
\hline
\end{tabular}

$$
\begin{aligned}
& \text { E0l }(G M) \\
& .5633-04(.320-05) \\
& .8560-02(.504-04) \\
& .046-03(.610-05) \\
& .0696-00(.163-03) \\
& .3966-00(.155-03)
\end{aligned}
$$

$E O L(A / 0)$

$1.0 L(A / 0)$
$0.1830(-350)$
$17.2730(.430)$
$2.3100(.050)$
$.2340(.0005)$

\begin{abstract}
EOL VIASS RATIO
1.0000

$.3349-00(.100-02)$

$.7885-01(.55 a-03)$

$.1282-01(.192-03)$
\end{abstract}

$70.0468(.0611)$

$23.4587(.0594)$

$5.5965(.0377)$

$.8980(.0127)$

E $L(G M)$

$1 \mathrm{SOICF}$

Fic

Fuctiol

Fuc +1

rotALS

$.37-0-02(.8438-04)$

$.12 \div 1-02(.2835-04)$

$.297-03(.7121-05)$

$.48 .9-04(.1292-05)$

$.5319-02(.1204-03)$

$\mathrm{BOL}(\mathrm{GM})$

$.3825-02(.1 ; 29-04)$

$.8275-03(.4041-05)$

$.1132-03(.6441-06)$

$.1130-04(.0165-07)$

$.4777-0<(.2400-04)$

F I S S TON S

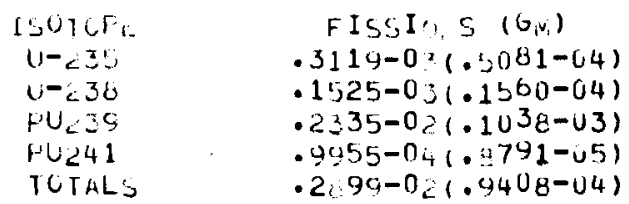

IURNIJP (WW:YMTM)

$$
\begin{array}{r}
299.4 \\
149.5 \\
2302.4 \\
28.7
\end{array}
$$

$2+58.7$

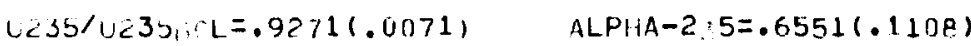
INPUT DAT:

CESIU -137 DECAYS $=.1288+1,(.2576+08) D / M / M L$

PUI OLCAYS $=.70,3+08(.1417+7) 0 / \mathrm{M} / \mathrm{ML}$

PERCEIIT DECAYS I PUC38=1:-70

SAMPLE T $T=19$. 3UUN $C L: D W T=2.837 G M$

Fố DL

PEKCE DT DECAYS, Y PUC38=1.70

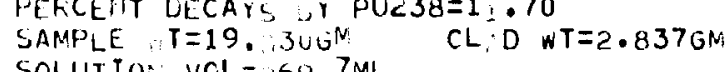

TIME OUT OF RTACTOR= .276\%RS

BOL PU241 TEC IYY CORRECTIO: TIME = .389YEA:S

CESIUM-137 II:-REACTOR DEC Y CORFECTION=1.0160

-- OAITITIES ENCLOSED BY PARENTHESES ARE ONE STANDARD DEVIATION ERRORS--.--
FSS 1 ON YIELO $.0626(.0014)$

$.062 \mathrm{~L}(.0060)$

$.0674(.0019)$
$.0660(.0033)$
NET PHODUCTIOIN (Gin)

$.6571-05(.320-05)$

$-.5162-03(.504-04)$

$.2046-03(.61 n-05)$

$-.3116-02(.163-03)$

$-.3428-02(.155-03)$
SPECIFIC ACTIVITY $(0 / \mathrm{M} / \mathrm{G}$ (M)

$.13616+12(.170+00)$

$.50130+12(.300+10)$

$.57600+10(.100+00)$

$.87000+10(.200+09)$

INET PRODUCTION (EN)

$-.1(150-03(.8655-64)$

$.4236-03(.2872-04)$

$.1865-03(.7150-0 b)$

$.3699-04(.1294-05)$

$.5421-03(.1228-03)$

$\begin{array}{cc}\text { DEL } & \text { MEV/FISSIOIN } \\ .0491(.0049) & 202 \\ .0563(.0056) & 209 \\ .0604(.0060) & 211 \\ & 214\end{array}$

PU239 FORIEED FROM U>38 $=.2976-02(.16-03)$
A! PHA-239= .3197(.0660)

\section{ASSUMPTIONS}

CESIUM- 137 HALF LIFE $=29.68(.10)$ YEARS

PU241 HALF LIFE $=14.5(0.5)$ YEARS

ALPHA241 $=.370(.030)$

PU241 HALF LIFE $=14.5(0.5)$ YEARS

ALPHA241 $=.370(.030)$ 

DISTR IBUTION

No. of

Copies

OFFSITE

1 AEC Chicago Patent Group

G.H. Lee

3 AEC Division of Reactor Development and Technology

Director, RDT

Chief, Water Reactors Branch

Chief, Reactor Physics Branch

3 AEC Division of Technical Information Extension

ONSITE

1 AEC Chicago Patent Group

R.K. Sharp (Richland)

$6 \quad \mathrm{RDT}$ Assistant Director of Pacific Northwest Programs

W.E. Fry

P.G. Holsted (2)

J.B. Kitchen

2 AEC Richland Operations Office

H.A. House

C.L. Robinson

45 Battelle-Northwest

N.E. Carter

D.E. Christensen

F.G. Dawson

J.C. Fox

M.D. Freshley

J.W. Kutcher

R.C. Liikala

R.P. Matsen (25)

D.R. Oden

L.C. Schmid

R.I. Smith

L.D. Willians

BNW legal (2)

Technical Information (5)

Technical Publications (2)

3 Batte1le Memorial Institute 
